To APPEAR IN The Astrophysical Journal Supplement

Preprint typeset using $\mathrm{LAT}_{\mathrm{EX}}$ style emulateapj v. 14/09/00

\title{
AN IMAGING SURVEY OF EARLY-TYPE BARRED GALAXIES
}

\author{
Peter ERWin \\ Instituto de Astrofisica de Canarias, C/ Via Láctea s/n, 38200 La Laguna, Tenerife, Spain \\ erwin@ll.iac.es \\ AND \\ LINDA S. SPARKE \\ University of Wisconsin-Madison, 475 North Charter Street, Madison, WI 53706 \\ sparke@astro.wisc.edu \\ To appear in The Astrophysical Journal Supplement
}

\begin{abstract}
This paper presents the results of a high-resolution imaging survey, using both ground-based and Hubble Space Telescope images, of a complete sample of nearby barred S0-Sa galaxies in the field, with a particular emphasis on identifying and measuring central structures within the bars: secondary bars, inner disks, nuclear rings and spirals, and off-plane dust. A discussion of the frequency and statistical properties of the various types of inner structures has already been published. Here, we present the data for the individual galaxies and measurements of their bars and inner structures. We set out the methods we use to find and measure these structures, and how we discriminate between them. In particular, we discuss some of the deficiencies of ellipse fitting of the isophotes, which by itself cannot always distinguish between bars, rings, spirals, and dust, and which can produce erroneous measurements of bar sizes and orientations.
\end{abstract}

Subject headings: galaxies: structure — galaxies: active — galaxies: elliptical and lenticular, cD — galaxies: spiral

\section{INTRODUCTION}

In the past decade, high-resolution images of the centers of disk galaxies have shown that many contain distinct, small-scale structures, such as inner bars, nuclear spirals, and nuclear rings. Buta \& Crocker (1993) present a compilation of data on nuclear rings, while Friedli (1996) reviews double-barred galaxies: those with a smaller, concentric bar inside the main bar. Infrared imaging of barswithin-bars (e.g., Shaw et al. 1993, 1995; Friedli et al. 1996; Jungwiert et al. 1997) has shown that the structures are not composed purely of dusty gas and young stars, but are also seen in the light of the old red stars that make up most of the disk mass.

As Buta \& Crocker point out, these features can be used to probe the galaxy dynamics; they argue that nuclear rings and spirals are linked to the inner Lindblad resonance of the large-scale bar or spiral pattern. The prevalence of small-scale stellar features tells us that the centers of many disk galaxies are dynamically "cool" enough to be responsive to gravitational effects on such small scales: most of the kinetic energy is in rotation, rather than random motions. A dynamically "hot" bulge, where the velocity dispersion $\sigma$ is of the same order as the rotation speed $V$, would not form bars or stellar rings. As Kormendy (1993) suggested, the bright inner regions of many galaxies may be dominated by "cool" disks, rather than "hot" bulges. When the inner features themselves contain significant mass, their non-axisymmetric gravitational pull affects the motions of stars and gas. For example, Shlosman, Frank, \& Begelman (1989) suggested that inner bars may be important in removing angular momentum from gas, feeding it into the center where it may fuel a starburst or active nucleus. Maciejewski et al. (2002) and Shlosman \& Heller (2002) have presented simulations of this complex flow.

To conduct a census of central structures, we observed a complete sample of nearby disk galaxies which were already known to contain a kiloparsec-scale main bar. Selecting $\mathrm{S} 0$ and Sa systems to minimize the effect of dust, we used the $3.5 \mathrm{~m}$ WIYN telescope, and archival optical and near-IR images from the Hubble Space Telescope (HST), to search for multiple bars and other central features ${ }^{1}$.

Our first results for three galaxies were presented in Erwin \& Sparke (1999); Erwin \& Sparke (2002, hereafter Paper I) discusses the types of structures that we found, their frequencies, and their relation to other properties of the galaxies. In this paper, we discuss our observations and the analysis techniques, and provide details and measurements for the individual galaxies. A key advantage of our approach is that we consider multiple types of inner structures (bars, disks, rings, spirals, and off-plane dust) and carefully distinguish between them. We also show that the common technique of fitting ellipses to isophotes, while quite useful, can often lead to erroneous measurements of bar parameters such as orientation and size.

\section{SAMPLE SELECTION}

To minimize the potential confusion from dust, we restricted ourselves to early-type disk galaxies; to maximize our chances of detecting small circumnuclear structures,

1 The WIYN Observatory is a joint facility of the University of Wisconsin-Madison, Indiana University, Yale University, and the National Optical Astronomy Observatories. Observations with the NASA/ESA Hubble Space Telescope obtained at the Space Telescope Science Institute, operated by the Association of Universities for Research in Astronomy, Inc., under NASA contract NAS5-26555. 
we chose large, nearby galaxies that were not close to edgeon. We selected all barred S0-Sa galaxies in the UGC catalog (Nilson 1973) which met the following criteria: declination $>10^{\circ}$, heliocentric radial velocity $\leq 2000 \mathrm{~km} \mathrm{~s}^{-1}$, major axis diameter $\geq 2^{\prime}$, and ratio of major to minor axis $a / b \leq 2$ (corresponding to $i \lesssim 60^{\circ}$ ). Galaxy types and axis measurements (at the $25 \mathrm{mag} \operatorname{arcsec}^{-2}$ level in $B$ ) were taken from de Vaucouleurs et al. (1991, hereafter RC3); radial velocities are from the NASA/IPAC Extragalactic Database (NED). Our use of the UGC and the restriction to galaxies with $D_{25} \geq 2^{\prime}$ means that the sample is biased in favor of high surface brightness galaxies; we believe that this is the only significant bias.

We excluded galaxies identified as members of the Virgo Cluster (those with VCC entries in NED), because the cluster environment may affect bar properties (e.g., Andersen 1996). In addition, galaxies in Virgo and the field with the same Hubble type may actually differ significantly in morphology: Koopman \& Kenney (1998) found that many Virgo Sa galaxies were more similar to field Sc galaxies than field Sa galaxies in terms of central light concentration.

Because our galaxies are selected on the basis of being optically barred - that is, SB or SAB according to RC3 - there is the possibility that we are missing some barred galaxies mistakenly classified as unbarred. Conflicting claims have been made about how much the bar fraction increases when galaxies are observed in the nearinfrared, where dust extinction is less of a problem; the most extensive survey to date is that of Eskridge et al. (2000), who classified 186 disk galaxies using $H$-band images. They found that the primary difference between the RC3 optical classifications and those from the near-IR was the increase in the relative number of strong (SB) bars; the total bar fraction (SB + SAB) increased by less than $10 \%$. This effect is weakest for early type galaxies: $65 \%$ of S0-Sab galaxies in their sample are barred according to the RC3, while $71 \%$ are barred in the near-IR. Since we restrict ourselves to $\mathrm{S} 0-\mathrm{Sa}$ galaxies, and select both SB and $\mathrm{SAB}$ classes, we are probably missing only one or two barred galaxies.

There is also the possibility that the barred appearance some of these galaxies at optical wavelengths is produced by, for example, strong dust lanes and star formation, rather than by a genuine bar in the underlying stellar disk. We restricted ourselves to early-type galaxies to minimize this problem. Nonetheless, three of the galaxies (NGC 2655, NGC 2685, and NGC 3032) show signs that they may not be barred; we discuss these cases individually in Section 5.

This sample has a total of 38 galaxies; their basic parameters, taken from the literature and from public databases - NED and the Lyon/Meudon Extragalactic Database (LEDA) - are listed in Table 1. Twenty of the galaxies are $\mathrm{S} 0$, ten are $\mathrm{S} 0 / \mathrm{a}$, and the remaining eight are $\mathrm{Sa}$; twenty-five are strongly barred (SB), with the remaining thirteen being SAB.

\section{OBSERVATIONS}

Table 2 summarizes the observations for each galaxy in the sample.

We observed all but two of the galaxies in the $B$ and $R$ bands at the $3.5 \mathrm{~m}$ WIYN telescope in Tucson, Arizona, between 1995 December and 1998 March. The detector was the S2KB CCD, a thinned $2048 \times 2048$ STIS chip with 21 -micron $\left(0.2^{\prime \prime}\right)$ pixels and an approximate field of view of $6.8^{\prime} \times 6.8^{\prime}$. Seeing - the mean FWHM of Moffat-profile fits to stars in $R$-band images - is given in Table 2; it ranged from $0.5-1.3^{\prime \prime}$, with a median of $0.8^{\prime \prime}$. Seeing in $B$-band images tended to be slightly worse $\left(0.7-1.65^{\prime \prime}\right)$. Most of the nights were not photometric, but since we were primarily interested in morphology and color variations, relative photometry is adequate for our purposes. In most cases, we used exposure times of $60 \mathrm{~s}$ and $300 \mathrm{~s}$ in $R$ and 120 s and 600 s in $B$. A few galaxy centers were bright enough to saturate the CCD in the $60 \mathrm{~s} R$-band exposures, so we made additional 30s exposures. For the two galaxies not observed with WIYN - NGC 936 and NGC $4314-$ reasonable ground-based images were available in the literature, and archival HST observations allowed us to carry out our analysis of their central regions. For NGC 936, we also obtained ground-based images of the central region in 2000 December with the $4.2 \mathrm{~m}$ William Herschel Telescope (La Palma, Spain). Finally, we used $B, R$, and $I$ images NGC 3729 from Tully et al. (1996), available via the NASA/IPAC Extragalactic Database (NED), to supplement WIYN images obtained under poor conditions.

Prior to mid-1996, an unrecognized nonlinearity plagued the S2KB CCD, dampening the response at levels $\lesssim 200$ counts above the bias (Ted von Hippel, private communication). This is a potential problem only for our $B$-band images, and then only for the outer disk regions. Two galaxies (NGC 2273 and NGC 3945) were observed both before and after the non-linearity was fixed; we found no significant differences in either the color maps or the ellipse fits. For images taken during the non-linear era, we treat color gradients in the outermost disk with skepticism; affected observations are noted in Table 2 .

Reduction of the images was carried out in standard fashion, using IRAF software. In a few cases where background sky gradients were present, we subtracted fitted backgrounds using the IMSURFIT task. Linear fits generally sufficed, though we were forced to use quadratic fits on one or two occasions. In images with no sky gradient, we measured the median sky levels in at least five regions far from the galaxy, and then subtracted the average of these values. To make color maps, $B$ and $R$ images were registered using stars in both images with the GEOMAP and GEOTRAN tasks, since simple linear shifts were not sufficient. We shifted the higher-resolution image to align with the lower-resolution image, then iteratively smoothed the shifted image until it matched the other's resolution. In all cases, we used the long-exposure images to make color maps, to maximize signal-to-noise. When the long $R$-band exposures had resulted in saturated galaxy centers, we made additional color maps using the shorter exposures.

HST data (see Table 2) were processed with the standard pipeline, and analyzed using tools in the STSDAS package within IRAF. Multiple exposures were combined with the STSDAS task CRREJ to eliminate cosmic-ray contamination; linear shifts were used to register images that were not aligned. The images were originally taken for a variety of programs, so filters and exposure times varied; 
in some snapshot survey images, the galaxy was off-center or saturated in the nucleus. Linear shifts sufficed to align WFPC2 images for making color maps; however, because all the galaxies are large enough to extend outside the WFPC2 field of view, we could not subtract the sky background. To make color maps using WFPC2 and NICMOS images, we rebinned the $\mathrm{PC} 2$ image to the corresponding NICMOS pixel scale, then shifted it to align with the NICMOS image. Fits of Moffat profiles to stars in WFPC2 and NICMOS images yielded consistent FWHM of $\approx 2.0$ pixels; pixel scales are $0.0455^{\prime \prime}$ pixel $^{-1}$ for the $\mathrm{PC} 2$ chip, $0.075^{\prime \prime}$ pixel $^{-1}$ for Camera 2 of NICMOS (NICMOS2), and $0.2^{\prime \prime} \mathrm{pixel}^{-1}$ for Camera 3 (NICMOS3).

\section{METHODS OF ANALYSIS}

Our primary goal in analyzing the images was the identification and measurement of central stellar and gaseous structures - as well as identifying galaxies which lack such features. Since we are interested in the relation of these features to the bars within which they are found, we also needed to measure the (main) bars in each galaxy. In this section, we discuss the techniques we used, what guidelines we used to discriminate among the various features we find, and how we measured them. Our measured values are listed in Table 5 .

\subsection{Techniques}

\subsubsection{Ellipse Fits}

We used ellipse fits to determine the position angle (PA) and ellipticity of galaxy isophotes as a function of radius. The specific algorithm was that of Jedrzejewski (1987), based partly on that of Carter (1978) and implemented in the IRAF task ELLIPSE (part of the STSDAS package). For each specified semi-major axis $a$, measured from the galaxy center, the program attempts to fit an ellipse to the isophotes of the galaxy; the center, position angle, and ellipticity $e=1-b / a$ (where $b$ is the semi-minor axis of the ellipse) of the ellipse are allowed to vary. The best results came from allowing ellipse centers to vary; this produced converged fits more often. We also employed iterative rejection of pixels with intensity values more than $3-\sigma$ from the mean along the ellipse, which minimizes contamination from cosmic rays, bad pixels, stars, and meteor/satellite tracks. We used logarithmic radial sampling with steps of 0.03 in scaled semi-major axis; sparser sampling can miss small-scale features and undersample rapid isophote twists, especially at large radii.

A good discussion of the ELLIPSE algorithm and the associated errors can be found in Rauscher (1995); he notes that its error estimates are likely to be overestimates. Both he and Jedrzejewski (1987) found that fitted ellipses are distorted for semi-major axis $a<3-5$ pixels, due to pixelization effects. Other distortions can come from limited resolution and atmospheric seeing, which make isophotes rounder in the center; focus problems with a few WIYN images produced elliptical distortions.

As an example of the effects of resolution, Figure 3 shows the results of fitting ellipses to both WIYN and WFPC2 images of the galaxy NGC 3412. Seeing for the $R$-band image was $\approx 1.2^{\prime \prime}$, while the mean FWHM of stellar profiles in the $\mathrm{PC} 2$ image was $\approx 0.1^{\prime \prime}$. There is no evidence for dust lanes in the central region of this galaxy (see Figure 20), so the differences in ellipticity for $a \lesssim 5^{\prime \prime}$ for the two images are due to the dramatic difference in resolution. At lower resolution, the point of maximum ellipticity shifts outward. Thus, estimates of the sizes of small-scale features based on the location of maximum ellipticity (see Section 4.3 below) can sometimes be exaggerated by poor seeing.

For a perfect fit to an isophote, the intensity along the ellipse would be constant. Variations in intensity along the ellipse can be expanded as a Fourier sum:

$$
I(\theta)=I_{0}+\sum_{n=3}^{4}\left[\tilde{A}_{n} \sin n \theta+\tilde{B}_{n} \cos n \theta\right]
$$

the first- and second-order coefficients are zero for a bestfit ellipse. The higher coefficients are divided by the local radial intensity gradient and by the ellipse semi-major axis, to obtain normalized coefficients of radial deviation $\delta r$ from a perfect ellipse, in a stretched coordinate system where the fitted ellipse is a circle with radius $r=(a b)^{1 / 2}$ :

$$
\frac{\delta r(\theta)}{r}=\sum_{k=3}^{4}\left[A_{k} \sin k \theta+B_{k} \cos k \theta\right] .
$$

The most commonly used coefficient is $B_{4}$, the $\cos 4 \theta$ term, which measures symmetric distortions from pure ellipticity. When $B_{4}>0$, the isophotes are pointed (lemonshaped) or "disky"; when $B_{4}<0$, the isophotes have a more rectangular or "boxy" shape. This term is related to the coefficient $a_{4} / a$ of Bender, Döbereiener, \& Möllenhoff (1988) by $a_{4} / a=\sqrt{b / a} B_{4}$.

In their study comparing SB0 galaxies to ellipticals with twisted isophotes, Nieto et al. (1992) argued that boxiness in ellipse fits is characteristic of bars, since the ends of bars in early-type galaxies often have a rectangular shape - see Athanassoula et al. (1990). However, an elongated, bisymmetric structure superimposed on rounder isophotes can also produce composite isophotes that are disky, with $B_{4}>0$. This effect is particularly strong in their images of UGC 2534, UGC 2708, and BGP 61; see also Figures 1 and 2 of this paper. For secondary bars, embedded more deeply inside bulges than primary bars, we find that the dominant sign of $B_{4}$ is almost always positive. For these reasons, $B_{4}$ cannot be used to discriminate bars from other embedded, elongated structures (such as disks or stellar rings). However, we do find it useful in some cases as confirmation that some central structure is present.

\subsubsection{Unsharp Masking}

Unsharp masking is a simple form of high-pass filtering, suppressing large-scale, low-frequency variations (e.g., Malin \& Zealey 1979; Malin, Quinn, \& Graham 1983). In contrast to the ellipse fits, this technique is sensitive to the details of low-amplitude, small-scale brightness variations. It can, for example, give a clearer view of a small central bar which may be outshone by a bright bulge, and of narrow lanes of obscuring dust. It is also particularly good for discriminating between structures which produce similar ellipse-fit features, such as bars and rings.

In origin, unsharp masking is a photographic technique for enhancing detail and contrast. For digital images, the procedure is as follows: make a smoothed copy of the original image, and then either subtract it from the original, or 
divide the original by it; the result is the "unsharp mask." A final, sharpened image is generated by either adding the (subtraction-generated) mask to the original, or multiplying the original by the (division-generated) mask; the mask can be scaled beforehand to control the amount of sharpening applied. Subtraction-based unsharp masking is the more common technique, since it is computationally faster.

We are interested in the mask itself, which directly displays the subtle, small-scale features we want to identify. In practice, division-generated masks have smaller dynamic ranges and are thus more easily viewed on the monitor, though the same features appear in both types of masks. (The subtraction method is more useful if the signal is near zero, as may be the case in the outer edge of a galaxy in a sky-subtracted image.) Detail on different spatial scales can be emphasized by changing the smoothing width: smoothing with a narrow Gaussian suppresses all but the smallest scales, while using progressively wider Gaussians allows larger scale variations to show up (though these then obscure the smaller-scale variations).

Figures $4 \mathrm{a}$ and $\mathrm{b}$ show how this process highlights smallscale variations, using a simple 1-D example. The lefthand panel is an $r^{1 / 4}$ intensity profile with a narrow Gaussian added. After dividing the original by the smoothed version, the Gaussian peak stands out as a sharp excess, bordered by shallow deficits, and the distracting radial gradient is largely gone. Figures $4 \mathrm{c}-\mathrm{f}$ show this process applied to an $R$-band image of the double-barred galaxy NGC 2950. Smoothing with a narrow Gaussian $(\sigma=5$ pixels) brings out the inner bar; using a wider Gaussian ( $\sigma=20$ pixels, panel f) emphasizes the outer bar, though it makes the small bar much harder to distinguish. Stars appear as bright peaks surrounded by dark "moats." In both masks, the ends of the bars show up as paired bright regions with dark regions just beyond, showing that the luminosity drops sharply there. Ohta, Hamabe, \& Wakamatsu (1990) noted that the ends of bars in early-type galaxies generally have sharp cutoffs in their luminosity profiles; our unsharp masks indicate that inner bars have a similar structure.

We generated unsharp masks with a range of smoothing, convolving the original images with Gaussians of $\sigma=2$, $5,10,20$, and 40 pixels (as a reminder, $\mathrm{FWHM}=2.354 \sigma$ for a Gaussian). The resulting masks were compared with isophotes, color maps, and the ellipse fits to help determine the nature and the orientation of various structures in the galaxies.

\subsubsection{Color Maps}

Color maps can be used to search for differences in the stellar population between the inner structures and their surroundings. They are also useful in delineating areas affected by dust, which are reddened, and recent star formation, where young blue stars are present. Nuclear rings are traditionally found as distinct red or blue ringlike features inside bars (e.g., Buta \& Crocker 1993). Because "raw" color maps can be dominated by photon noise, we smoothed them with median filters. Minimal filtering (using a median box of width $w=5$ pixels) is effective at cleaning up moderately strong color contrasts. Higher levels of smoothing are useful for finding very smooth, large- scale color features and gradients.

We note that when the dust extinction is high enough to render the dust opaque at optical wavelengths $\left(A_{V}>1\right)$, our $B-V$ color maps will not probe the true extinction, since only the foreground, unreddened stars will be visible.

\subsection{Identifying, Naming, and Discriminating Features}

Our basic approach was to look for distinct features in ellipse fits - particularly peaks in the ellipticity. This is based on the approach of Wozniak et al. (1995, hereafter W95), who looked for bars by finding local ellipticity maxima, accompanied by stationary position-angle values.

However, there can be cases where a bar or other feature is present but does not appear as a maximum in ellipticity; so we considered any significant deviation in the ellipse fits as a possible structure. For example, projection effects cause the bar of NGC 4143 (Figure 26) to manifest itself as a strong twist in position angle without an obvious ellipticity peak; the inner bar of NGC 3945 is another example (see Erwin \& Sparke 1999, Figure 2). NGC 2962's inner bar (Figure 17) is only apparent in the fits as an ellipticity minimum, with a very modest change in position angle; in this case, the $B_{4}$ coefficients clearly indicate disky isophotes, and unsharp masks confirm the presence of a bar). Similarly, both Nieto et al. (1992) and Busarello et al. (1996) identified the main bar in NGC 3384 with a minimum in the ellipticity.

Finally, we used unsharp masks, color maps, and examination of the original images to evaluate all such features and determine when they were most likely due only to dust lanes or star formation, to discriminate between structures such as bars, rings, and spirals, (which may all have similar ellipse-fit signatures), and to identify features not apparent in ellipse fits (such as some nuclear rings and off-plane dust lanes).

For galaxies with two (or more) bars, we adopt the terminology of Friedli \& Martinet (1993) and W95: the larger bar is the primary, outer, or main bar, and the smaller, embedded bar is the secondary, inner, or small bar. In NGC 2681, which has three bars, the third, innermost bar is the tertiary bar. In galaxies with only one bar, we usually refer to it as simply "the" bar.

There is some confusion in the literature as to what constitutes a "nuclear" ring versus an "inner" ring. We use the scheme of Buta (e.g., Buta \& Crocker 1993; Buta 1995), which uses the bar as a measuring stick. Outer rings lie well outside the bar, usually at slightly over twice the bar radius. Inner rings lie just beyond the ends of the bar, while nuclear rings are found inside the bar. We use the primary bar as the referent, so a nuclear ring in a doublebarred galaxy is one inside the primary bar. (We found no clear cases of rings inside secondary bars, which lets us avoid the difficult question of what to call such things.)

Disks residing inside bars are called inner disks. The alternative, "nuclear disk," has generally been used in the literature for the very small-scale disks (sizes $\lesssim 1^{\prime \prime}$ and $\lesssim 100$ pc) revealed by HST in ellipticals and edge-on S0's (e.g., Scorza \& van den Bosch 1998). The larger disks found by Seifert \& Scorza (1996) have physical and angular sizes roughly the same as our disks and secondary bars, so we adopt their term.

The specific features that we list in Table 5 are: 
- Primary bars. Since our galaxies are classified as $\mathrm{SB}$ or $\mathrm{SAB}$, they should in principle all have at least one bar. We looked for ellipticity peaks or position angle twists in the ellipse fits, and based our measurements on those, but differ from W95 in that we did not require constant PA for a bar. The ellipticity maximum for NGC 3412's bar (Figure 3) is a local maximum within a larger-scale minimum; both here and in Figure 2, the position angle twists to a maximum or minimum value without staying constant over a large range of semi-major axis. We inspected color maps, unsharp masks, and the original images to check that strong dust lanes, star formation, rings, or spiral arms were not responsible for features in the ellipse fits, and to ensure that we measured the bar itself (as opposed to, e.g., spiral arms outside the bar).

- Secondary bars and inner disks. We usually identified these as local ellipticity peaks or distinct PA twists; in certain cases, ellipticity minima were also an indication. As with primary bars, we examined color maps, unsharp masks, and the original images to ensure that strong dust lanes, star formation, nuclear rings or spirals were not the cause. We distinguished inner disks by requiring that the PA differ from that of the outer disk by less than $10^{\circ}$, and that the maximum ellipticity be less than that of the outer disk. Figure 2 provides a clear example of a secondary bar, while Figure 3 shows an inner disk. The inner-disk class is somewhat ambiguous: a secondary bar fortuitously aligned with the projected outer disk would be called an inner disk, unless it appeared more elliptical than the outer disk; highly flattened inner bulges could also be detected and classed as inner disks. Our secondary bars almost always display a distinct appearance in unsharp masks (e.g., Figure 4), while our inner disks generally do not, so we believe that these are distinct populations. Paper I presents statistical evidence that these are indeed distinct classes.

- Nuclear rings. We recognize four general classes of nuclear ring. Star-forming nuclear rings are readily apparent in images as a ringlike pattern of bright knots, usually intermixed with tightly wrapped spiral dust lanes; in color maps, the knots are blue. The well-studied nuclear ring of NGC 4314 (Figure 30) is an excellent prototype. Blue nuclear rings are smooth, blue features in color maps; they lack the uneven, knot-like appearance of star-forming rings, and have little or no dust in the ring. Dusty (red) nuclear rings are seen in color maps as red features without any blue knots; they can also be identified in unsharp masks as dark rings. Finally, stellar nuclear rings show up as ellipticity peaks, and were distinguished from bars and disks by their appearance in unsharp masks; Erwin \& Sparke (1999) present two clear examples. They seem to have no clumps of dust or young stars, and thus do not stand out in color maps.
- Inner and Outer Rings. We did not always identify or measure these, but in some cases it can be unclear from casual inspection of the ellipse fits which peak corresponds to the (primary) bar, so it is helpful to clearly distinguish bars from exterior rings. Sometimes it is difficult to define the end of the bar from the ellipse fits; if an inner ring is present, a measurement of its size (from color maps or unsharp masks) might provide a useful upper bound to the bar size. Finally, some galaxies have strong outer rings which obscure the true orientation and ellipticity of the outer disk; the shapes and orientations of the inner and outer rings are then useful to help constrain the disk inclination and PA (see Section 4.2.1, below).

In addition, we found examples of nuclear spirals and off-plane gas. The former are distinct regions of spiral structure surrounding the nucleus, usually identifiable in unsharp masks or color maps or both; we typically observed multiple dust lanes rather than distinct stellar arms. We use the term "off-plane gas" somewhat loosely to identify galaxies where at least some of the gas appears to be off-plane with respect to the stellar disk. The "Helix Galaxy" NGC 2685, with dust and stellar rings surrounding the central regions, is a canonical example. For an off-plane gas classification, we also required at least some corroborating kinematic evidence - e.g., H I maps from the literature which indicate that some gas is in orbits inclined with respect to the stellar disk.

We assumed that spiral arms trail, and used the orientation of any stellar or dusty spiral arms to derive the direction of disk rotation, as listed in Table 5.

\subsubsection{The Problem of Inclination and Deprojection}

The sizes and orientations of structures reported in Table 5 are measured values - i.e., without deprojection. Some authors deproject galaxy images and then perform isophote fits (e.g., W95). But deprojecting digital images can create artifacts in the central regions due to the interpolation of pixel values: tests by Jungwiert et al. (1997) on artificial images showed that fitting ellipses to a deprojected image can produce spurious results, including false signals of secondary bars. An alternative approach is to deproject the fitted ellipses analytically, as done by Jungwiert et al. and Laine et al. (2002). While this avoids interpolation problems, it is vulnerable to the fact that ellipses are not always good fits to galaxy substructures. For example, we find that the position angles of ellipses can be misaligned with respect to the bars they are ostensibly fitting; this is especially true for inner bars, where we find deviations of $10-80^{\circ}$, but it can also occur for outer bars (see Section 4.3 and Figures 5 and 6). Deprojecting these ellipses will then give misleading values for both position angles and sizes of bars. Finally, two-dimensional deprojection, whether of images or fitted ellipses, only works if all structures in the galaxy are coplanar and flat. Since we are interested in structures with sizes comparable to, or smaller than, the central bulge, this is not a safe assumption to make.

Nevertheless, we do want to compare the (deprojected) lengths and orientations of bars and other structures which 
we presume lie in the plane of a galaxy's disk, so we did attempt to determine the inclination and orientation of the disk of each galaxy. This was also necessary for our innerdisk classification (above). We generally assumed that the outermost isophotes that we could measure are those of an intrinsically circular disk. The inclination $i$ to the line of sight $\left(i=0^{\circ}\right.$ for a face-on disk, and $90^{\circ}$ for edge-on $)$ is then

$$
\sin i=\sqrt{\frac{2 e-e^{2}}{1-q_{0}^{2}}},
$$

where $e$ is the outer disk ellipticity and $q_{0}$ is the intrinsic (edge-on) disk axis ratio (Hubble 1926); we assumed an average value of $q_{0}=0.2$ (Lambas, Maddox, \& Loveday 1992).

Several galaxies have strong, "detached" outer rings, with no detectable disk beyond; the "outer ring" then is the largest listed component in Table 5. In these cases, the outer isophotes may not be those of an intrinsically circular structure: Buta $(1986,1995)$ has shown that outer rings have typical axis ratios $\sim 0.8$, and are either perpendicular to, or parallel with, the bar. The luminous region interior to the ring, surrounding the bar, may not be circular either, since the bars can be surrounded by intrinsically elliptical lenses and inner rings, aligned with the bar (Kormendy 1979; Buta 1986). In such cases, we could only derive a range of possible inclinations, by combining the statistical results of Buta $(1986,1995)$ with kinematic information from the literature. In particular, we assumed that the circular velocity $100 \mathrm{~km} \mathrm{~s}^{-1} \leq V_{\text {circ }} \leq 500$ $\mathrm{km} \mathrm{s}^{-1}$. The upper limit is the highest known value for a disk galaxy (UGC 12591, Giovanelli et al. 1986), while $100 \mathrm{~km} \mathrm{~s}^{-1}$ is a lower limit to the inclination-corrected circular velocities measured for $18 \mathrm{~S} 0$ and $\mathrm{S} 0 /$ a galaxies by Neistein et al. (1999). Details of this process are given in the discussions of individual galaxies, below.

\subsection{Measuring Orientations and Sizes}

For each feature identified in Table 5, we measured its semi-major axis, position angle, and ellipticity. For the outer disks we list $R_{25}$, taken from RC3.

Position angles (counter-clockwise from north) were measured from the ellipse fits, unless unsharp masking and direct inspection of the isophotes indicate a significantly different value (see below). Using the ellipse fits, we found the point where ellipticity reaches a local maximum $\left(e_{\max }\right)$ at semi-major axis $a_{\max }$. The position angle at $e_{\max }$ is often a good indication of the structure's position angle, though dust lanes and spiral arms can confuse the issue. In some cases, the PA curve reaches a local minimum or maximum near $a_{\text {max }}$; this extremal value is then a reasonable estimate for the PA of the bar's major axis.

However, we find that the orientation of the fitted ellipses often does not match that of the galaxy components involved. This is a problem particularly for secondary bars embedded in bright bulges: unless the galaxy is close to face-on, or the bulge nearly spherical, the projected isophotes of the bulge will be elliptical, and thus the combined bulge + bar isophotes may not accurately reflect the bar's orientation. Figure 5 illustrates cases where the inner isophotes of double-barred galaxies show pointed (disky) deviations - and the unsharp masks show the secondary bar structure - at a different position angle from what the ellipse fits report. This is the case for about half the secondary bars in our sample; the differences in position angle range from $10^{\circ}$ to $80^{\circ}$. In such cases, we measured the bar's PA from the unsharp mask and the isophotes, and report the PA from the ellipse fits in parentheses. We found similar, smaller deviations for twelve primary bars (e.g., Figure 6); see Table 5. Busarello et al. (1996) found a similar discrepancy for NGC 3384, where the main bar's PA measured with an adaptive Laplacian filter (similar to an unsharp mask) differed by $60^{\circ}$ from the ellipse-fit value.

Unfortunately, there are no standard methods for measuring the sizes of bars. One fairly common measure is $a_{\max }$ (e.g., Wozniak \& Pierce 1991; Regan \& Elmegreen 1997; Jungwiert et al. 1997; Laine et al. 2002). However, W95 argued that that in at least some galaxies the isophotes remain elongated, parallel, and "bar-shaped" beyond $a_{\max }$. For small features, this measure can also be sensitive to seeing, as we pointed out in Section 4.1.1. For an upper limit to bar length, W95 used the point outside $a_{\max }$ where ellipticity reaches a local minimum, at semimajor axis $a_{\mathrm{min}}$. But this can also give misleading results if, e.g., there are strong dust lanes, spiral arms or a ring starting near the ends of the bar. In these cases the isophotal ellipticity can remain high even though the ellipses are no longer tracing the bar.

Our alternate method is based on the fact that bars in early-type galaxies are extremely straight, deviating from a constant position angle by less than $\pm 2^{\circ}$ (Ohta et al. 1990). A plausible upper limit for bar length, $a_{10}$, is the point where the PA of the ellipses has changed by $10^{\circ}$ from the bar's PA. This radius is somewhat sensitive to viewing angle, and the technique fails completely if the bar happens to be closely aligned with the disk major axis. So we used the smaller of $a_{\text {min }}$ and $a_{10}$ as the length of the bar.

In Table 3 we compare measurements of bar length for four well-studied SB galaxies. For these galaxies, bar length has been determined by: decomposition into disk, bulge, and bar components (Kent \& Glaudell 1989); analysis of how azimuthal bar-interbar contrast varies with radius (Ohta et al. 1990); or Fourier decomposition (Quillen, Frogel, \& González 1994). Measurements apparently made by eye are also included for two of the galaxies. For three of the four galaxies, $a_{10}$ is a better match to the published bar lengths than either $a_{\max }$ or $a_{\text {min }}$. However, for NGC 4314 the published lengths are all closer to $a_{\max }$. Consequently, we list both measurements for bars and disk/bar candidates in Table 5: $a_{\max }$ and the smaller of $a_{10}$ and $a_{\min }$.

\section{RESULTS FOR INDIVIDUAL GALAXIES}

In this section we discuss the individual galaxies in the sample, and present figures for each (Figures 7-42). These figures show the ellipse fits, contour maps, and one or more color maps or unsharp masks.

Isophote maps use the square root of logarithmic (skysubtracted) counts for the ground-based $R$-band images. HST images images are not sky-subtracted, since most of the galaxies are larger than the WFC field of view, but use the same scaling; DSS images, which are taken from photographic plates, are plotted with linear contours.

Ellipse fits to the ground-based $R$-band images are plotted in black; those for HST images are plotted in gray (red 
for online figures). The longest-wavelength HST image available is used for the ellipse fits, to minimize confusion due to dust and star formation. We label major features in the ellipse fits as follows: "B" = bar, "D" = inner disk, "NR" = nuclear ring, "IR" = inner ring, and "OR" = outer ring.

Color maps are displayed as grayscale images (color in the online figures). Lighter values are redder. (For the online figures, we use a natural color map, with red = red and blue = blue.) The displayed colors are adjusted for each plot to maximize contrast; the range in the appropriate astronomical color index is given in the figure captions for each galaxy. Because our images are uncalibrated, these are relative color maps. The applied median smoothing $w$ is given in pixels. Unsharp masks are displayed as grayscale images, with excesses light and deficits dark. The smoothing $\sigma$ is given in pixels.

Two of the galaxies from our sample - NGC 2681 and NGC 3945 - have already been discussed in Erwin \& Sparke (1999). The reader is referred to that paper for detailed analyses and figures of those galaxies, though we include some additional notes for each below.

\subsection{NGC 718: Double bar, nuclear ring}

Figure 7: This weakly barred galaxy has two strong, uneven spiral arms outside the bar, with the western arm both stronger and bluer than the eastern arm; Kennicut \& Edgar (1986) concluded that the western arm was the site of recent star formation. The two arms may form a weak outer pseudo-ring, oriented at $\mathrm{PA} \approx 45^{\circ}$, which is the value for the disk given in RC3; our disk orientation $\left(P A=5^{\circ}\right)$ is based on isophotes further out.

The color map shows several strong dust lanes within the bar, only one of which is a plausible leading-edge lane. There is also a blue ring oriented roughly perpendicular to the bar; the southeast side of the ring is redder, probably due to the nearby dust lane. This is one of two examples of a "blue nuclear ring" in our sample: it is quite smooth, with no signs of dust or knots which might indicate current star formation. In fact the ring is $\sim 0.1$ mag bluer than most of the outer disk, but $\sim 0.1-0.15$ mag redder than the strong, blue western arm, so it could be fairly old. Nonetheless, due to its distinct color we consider it a "young" and presumably gas-rich nuclear ring. The ring does not show up in unsharp masks, except as a possible enhancement surrounding the inner bar in the $R$ band, at the inside edge of the blue ring. (The lower signal and low resolution of the $B$ image $-1.7^{\prime \prime}$ - may explain why the ring does not appear in $B$-band unsharp masks.)

A secondary bar shows up as an ellipticity peak, and in the $\sigma=2$ unsharp mask. The position angle we report for the inner bar is the value at $a_{\max }$, which agrees with the elongated feature in the unsharp mask. Telescope focus problems caused the elliptical shape of the nucleus in the unsharp mask, and the inward twist in ellipse-fit PA. Additional images obtained in 2000 December with the William Herschel Telescope's Auxport camera (see next galaxy) confirm the presence of the inner bar.

\subsection{NGC 936: Nuclear ring}

Figure 8: This is a very well studied, prototypical SB0 galaxy (e.g., Kormendy 1983; Kent \& Glaudell 1989;
Merrifield \& Kuijken 1995). To supplement the archival WFCP2 image (short exposures in the F555W filter), we obtained high-resolution, ground-based images of the bar region with the Auxiliary Port Camera of the $4.2 \mathrm{~m}$ William Herschel Telescope (a $1024 \times 1024$ CCD with $0.11^{\prime \prime}$ pixels). These show an elliptical feature with semi-major axis $\approx 8^{\prime \prime}$; unsharp masking (panel $\mathrm{f}$ ) reveals this to be a nuclear ring, with orientation and ellipticity roughly consistent with a circular ring seen in projection. Because this appears equally well in $B, V$, and $R$ images, and does not appear as a distinct feature in color maps (not shown), we conclude that this ring is predominantly stellar and probably similar in population to the surrounding stars of the bulge and bar.

The ring does not show up in unsharp masks of the WFPC2 images (panel e), although the ellipse fits do show a similar peak. By comparing unsharp masks of long (300s) and short (60s) WHT exposures, we have determined that high signal/noise is necessary to see the ring clearly in unsharp masks: it is barely detectable in 60s exposures, but clearly visible in 300s. Since the combined WFPC2 long exposures total only 280s, the difference in mirror and pixel sizes means the 300 s WHT images receive $\sim 20$ times the signal per pixel of the combined HST images (since this is the bright central region, the lower sky background of HST images has little effect on $\mathrm{S} / \mathrm{N}$ ).

Both the WHT and WFPC2 images show a weak linear dust feature approximately $4.5^{\prime \prime}$ long, about $3^{\prime \prime}$ northnortheast of the nucleus at closest approach, curving slightly inward at the west end. An even fainter straight dust lane extends from the nucleus to the east-northeast, about $0.8^{\prime \prime}$ long; this may be responsible for the innermost $\left(a<1^{\prime \prime}\right)$ features in the WFPC2 ellipse fits.

\subsection{NGC 1022: Dust-obscured}

Figure 9: This is a known starburst galaxy (e.g., Devereux 1989; Usui et al. 1998). The roundness of the outer isophotes and the low $\mathrm{H}$ I width $\left(W_{50}=182 \mathrm{~km} \mathrm{~s}^{-1}\right)$ reported by Theureau et al. (1998) both indicate the galaxy is close to face-on; the position angle for the outer disk is rather uncertain.

Unsharp masks suggest a faint outer ring with $\mathrm{R}_{1}^{\prime}$ morphology and an inner pseudo-ring surrounding the bar. The latter ring also shows up as a red feature in the color maps, and as an ellipticity minimum at $a \sim 30^{\prime \prime}$. The bar itself has notably blue ends, suggesting recent star formation there. Within the bar, strong obscuring dust lanes are visible, especially in the northwest half; these prevent us from determining what stellar structures might exist inside the bar.

\subsection{NGC 2273 (Mrk 620): Nuclear spiral, nuclear ring}

Figure 10: This is a rather spectacular four-ringed spiral galaxy, with two outer rings, an inner ring, and a bright star-forming nuclear ring with a luminous blue nuclear spiral inside. The latter structure masquerades as a secondary bar in ellipse fits and ground-based images.

The two outer rings make determining the disk inclination and position angle somewhat difficult. Fortunately, the H I mapping performed by van Driel \& van Woerden (1991) yields plausible values of $50^{\circ}$ for both inclination and position angle for the two outer rings, where most 
of the $\mathrm{H} \mathrm{I}$ is concentrated. We assume these rings to be coplanar with the stellar disk.

The outer of the two outer rings is blue, as is the inner ring which wraps around the bar. Blue regions at the end of the bar are probably associated with the H II regions found by González Delgado et al. (1997). The color maps show leading-edge dust lanes, and strong curving lanes in between the nuclear region and the ends of the bar that are particularly strong examples of what Sheth et al. (2000) term "dust spurs." The combination of leadingedge lanes, which are strongest to the north and south of the galaxy center, and the spurs are probably responsible for the $r \sim 7^{\prime \prime}$ red ring seen in the low-resolution optical color maps of Yankulova (1999).

Mulchaey, Regan, \& Kundu (1997) suggested there was a secondary bar, based on ellipse fits to their ground-based near-IR images and the appearance of the images. We disagree. Our ellipse fits do agree with theirs - in fact, the NICMOS2 (F160W) fits show a distinct ellipticity peak, accompanied by a sharp twist in position angle to a minimum value which is not that of the outer disk. But the WFPC2 and NICMOS images show an $r \approx 2^{\prime \prime}$ nuclear ring with a spiral inside, and not a secondary bar. This combined structure can also be seen in the WFPC2 images presented by Ferruit, Wilson, \& Mulchaey (2000). The ring is luminous and blue, and its irregular, patchy character suggests recent star formation. Ferruit et al.'s [N II] $+\mathrm{H} \alpha$ image shows emission from the ring, with $\mathrm{H} \alpha$ : [O III] ratios characteristic of $\mathrm{H}$ II regions, so we class this as a star-forming ring. The spiral, which appears to be the cause of the innermost ellipticity peak, consists of spiral dust lanes and a luminous, two-armed blue spiral (compare panels e and f); its size and position angle of $20^{\circ}$ also matches the extended amorphous $20 \mathrm{~cm}$ radio emission (Ulvestad \& Wilson 1984). The molecular "bar" seen in the intermediate resolution $\left(3.0^{\prime \prime} \times 2.5^{\prime \prime}\right) \mathrm{CO}$ maps of Petitpas \& Wilson (2002) is probably the gas counterpart of this ring + spiral structure.

A blue spiral arm outside the ring on the west side (at the right-hand edge of panels f; see also the F547M image in Ferruit et al.) is reminiscent of the blue spiral arms outside the nuclear ring in NGC 4314 (see below). A fainter counterpart may be present on the east side.

The galaxy has a Seyfert 2 nuclear spectrum, with broad $\mathrm{H} \alpha$ emission detected in polarized light by Moran et al. (2000). In the HST images, the nucleus is blue and luminous, elongated east-west, parallel to the east-west doublenucleus radio structure seen by Ulvestad \& Wilson and Nagar et al. (1999). We may be seeing the strong [O III] 5007 emission noted by Ferruit et al. (2000) in their WFPC2 images, since the F606W filter we used for our color maps is wide enough to let in [O III] emission.

\subsection{NGC 2655: Dust-obscured, off-plane gas}

Figure 11: This is the largest, most luminous galaxy in the sample, with both an active nucleus and substantial off-plane gas. The isophotes are nearly round, though the outer disk is irregular. We measure the PA and inclination at the ellipticity minimum at $a \sim 110-130^{\prime \prime}$. The central elliptical region bears little resemblance to any of the other galaxies in our sample; this galaxy may not, in fact, be barred.
We find two sets of dust features in this galaxy: faint, outer, counter-clockwise spiral arms, which appear on all sides of the galaxy for $r \gtrsim 50^{\prime \prime}$, and much stronger, disorganized lanes closer to the center, almost entirely on the west side of the galaxy. The kinematics of the extended, irregular H I gas (Huchtmeier \& Richter 1982; Shane \& Krumm 1983) indicates significant off-plane gas. We show our H I map (van Moorsel et al. 2002) in panel e of the figure.

WFPC2 images (Barth et al. 1998) show that the strong, one-sided dust lanes continue into the nuclear regions, so we do not plot ellipse fits to the HST data.

\subsection{NGC 2681: Triple bar, nuclear ring}

This triple-barred galaxy is discussed by Erwin \& Sparke (1999); updated measurements for the secondary and tertiary bar can be found in Table 5 . The WIYN $B-R$ color map and unsharp masking of the PC1 image show curved dust lanes within the secondary bar, along the leading edges. The innermost visible lanes appear to form a spiral pattern just outside the tertiary bar; the strong diffraction pattern in the PC1 image from the (saturated) nucleus prevents us from determining whether there are dust lanes within the tertiary bar. Unsharp masking at the lowest level $(\sigma=2)$ of the NICMOS3 F160W image shows that the tertiary bar may be bounded by a circular, stellar nuclear ring. Since this is at the edge of detection, we do not list it in Table 5 .

\subsection{NGC 2685: Inner disk, off-plane gas}

Figure 12: This the famous "Helix" or "Spindle" Galaxy, a canonical polar ring system. The classification (R)SB0 ${ }^{+}$- is apparently based on the combination of the outer optical ring and the inner elliptical structure (the "spindle"). However, stellar velocity curves along the spindle (Schechter \& Gunn 1978; Sil'chenko 1998) show strong rotation, and these authors conclude that the spindle is an S0 disk, seen nearly edge-on. If that is so, then the outer ring lies in a different plane, though its $\mathrm{H} I$ has the same sense of rotation as the stars (Schechter \& Gunn 1978; Mahon 1992). Because of the extreme uncertainty about the galaxy's orientation, we cannot determine its inclination. We take the position angle for the line-of-nodes as that of the outer ring and the spindle; since the inner disk (see below) has the same alignment, deprojection would not change its size.

The spindle appears more elliptical in the near-IR than it is in the optical (panel b of the figure), as noted by Peletier \& Christodoulou (1993). This is undoubtedly because the strong polar dust lanes crossing over the NE side of the spindle make the optical isophotes rounder. The NICMOS3 image shows an inner ellipticity peak, aligned with the spindle, which also appears in the ground-based images of Peletier \& Christodoulou. Unsharp masks (e.g., panel f) show an elliptical feature with no bar-like characteristics, so we are fairly confident this is an inner disk; Ravindranath et al. (2001) noted the presence of this disk after subtracting a 2-D Nuker-law fit to the inner regions of the NICMOS image. The inner disk shows up in the color map as an elliptical red feature with $a \sim 2^{\prime \prime}$, parallel with the spindle. There is a color gradient within the disk, with the center about 0.2 mag redder in $B-R$ than the 
edge.

In the color maps, the spindle is dominated by the polar rings, seen as red arcs crossing over the brightest part of the spindle and blue outside (e.g., the blue arc near the western edge of panel d). These luminous rings have been mapped in both H I and CO (Shane 1980; Mahon 1992; Watson, Guptill, \& Buchholz 1994) and are sites of star formation (Eskridge \& Pogge 1997, e.g., ). Faint red and blue lanes in the region between the spindle and the outer ring (panel c) do not appear connected with the polar rings.

\subsection{NGC 2787: Dust-obscured, inner disk, off-plane gas}

Figure 13: This galaxy is notable for both a large inner disk and a dramatic, off-plane dust disk in the central regions. Although the latter obscures the inner $r \lesssim 10^{\prime \prime}$, the inner disk is large enough to be seen outside the obscuration. (Unfortunately, the NICMOS3 images are severely off-center and thus not usable.)

H I mapping by Shostak (1987) showed that the neutral gas in this galaxy is distributed in a ring with diameter $=6.4^{\prime}$, inclined (at either $25^{\circ}$ or $84^{\circ}$ ) with respect to the stellar disk; the gas distribution's line of nodes is at a position angle of $\approx 140^{\circ}$, in contrast to the position angle of $109^{\circ}$ which we measure for the stellar disk.

The ellipse fits show peaks in ellipticity due to the outer disk, the bar, the bar, and a large inner disk (the latter is also apparent in $\sigma=40$ unsharp masks, not shown here). There are additional, stronger ellipticity peaks at $a \approx 5^{\prime \prime}$ and (in the HST ellipse fits) $a \approx 6.5^{\prime \prime}$; these are both due to the strong dust lanes near the center (see below).

The ground-based $B-R$ color maps show disturbed, asymmetric patches of dust. A large lane seems to cross over the northwest side of the disk (panel c), and might be associated with some of the tilted $\mathrm{H}$ I. There are fainter, fairly linear lanes in the bar-inner disk region, none of which appear to be leading-edge lanes of the bar. Finally, there is a broad arc of dust surrounding the center (panel d).

In the HST images, this near-nuclear dust is clearly resolved into a spectacular set of concentric, elliptical dust rings, covering a radial range of $\sim 1.5-10^{\prime \prime}$ (previously noted by Sarzi et al. 2001). The rings have a common position angles of $\approx 40^{\circ}$ and an ellipticity $\approx 0.43$ (best measured from the innermost rings, panel e), indicating an inclination to the line of sight of about $55^{\circ}$ if they are intrinsically circular. Tracing the degree of extinction suggests a line-of-nodes of $\sim 70^{\circ}$ for the rings' intersection with the stellar disk of the galaxy. Careful inspection of the isophotes shows that essentially all of the variation in the ellipse fits for $r \lesssim 10^{\prime \prime}$ is due to extinction by this dust.

There is a central region almost free of dust, with a distinctly blue nucleus; this nucleus is the bluest part of the PC image (about 0.2 magnitudes bluer in $V-I$ than the region outside the dust lanes). This is probably the active nucleus (broad $\mathrm{H} \alpha$ emission was seen by $\mathrm{Ho}$ et al. 1997); Sarzi et al. (2001) recently found evidence for a central black hole with mass $\sim 10^{8} M_{\odot}$.

\subsection{NGC 2859: Double bar, nuclear ring}

Figure 14: This is our strongest example of a double barred galaxy; the secondary bar is readily apparent in the isophotes (e.g., panel e of the figure), and was noted by Kormendy (1979) and W95.

The $B-R$ color map shows a faint leading-edge dust lane in the eastern half of the primary bar, curving in toward the central regions. There, it appears to join a dusty nuclear ring which surrounds and is aligned with the secondary bar; unsharp masking $(\sigma=5$, not shown here) indicates faint spiral dust lanes just outside of the nuclear ring and merging with it, on both the north and south sides. The secondary bar shows up as an elongated red feature in the color maps, but is clearly asymmetric in color. This suggests that the color difference is caused by dust in the secondary bar, rather than by a different stellar population.

NGC 2859 also has a very clear example of a detached outer ring, which makes determining the galaxy's inclination and PA difficult. The $\mathrm{H}$ I profile width of $W_{50} \approx 170$ $\mathrm{km} \mathrm{s}^{-1}$ (Wardle \& Knapp 1986; Burstein, Krumm, \& Salpeter 1987) permits $10^{\circ}<i<58^{\circ}$ for $100<V_{\text {circ }}<$ $500 \mathrm{~km} \mathrm{~s}^{-1}$. Since the maximum rotation velocity in Kormendy's (1982) optical spectrum at PA $=90^{\circ}\left(86 \mathrm{~km} \mathrm{~s}^{-1}\right)$ is very close to half of $W_{50}$, the galaxy's line of nodes is probably not far from $90^{\circ}$. From here, we can use the shapes of the outer ring and lens to further constrain the inclination. An intrinsically round outer ring would give $i=40^{\circ}$; however, if it has a typical axis ratio of 0.8 and is perpendicular to the bar, then $i=15^{\circ}$. The lens, outlined by a dusty inner ring in the $B-R$ color map (panel d), is seen with $e \approx 0.10$ at $a \approx 50^{\prime \prime}$, elongated parallel with the bar; this implies $i<39^{\circ}$, for an upper limit of 0.7 on the lens's intrinsic axis ratio, and $i=27^{\circ}$ for a typical lens axis ratio of 0.8 . We adopt $i=25^{\circ}$, and $\mathrm{PA}=90^{\circ}$ for the line-of-nodes.

\subsection{NGC 2880: Inner disk}

Figure 15: This relatively featureless galaxy was the subject of extensive long-slit spectral analysis by Munn (1992), who found a moderate metallicity gradient and no evidence for emission lines.

The weak bar shows up mainly as a pronounced twist in position angle; the position angle we measure, based on the isophotes and unsharp masks, differs by more than $20^{\circ}$ from that given by the ellipse fits. Unsharp masking (panel d) shows evidence for a partial ring just outside the bar. Interior to the bar is a small ellipticity peak with a position angle very close to the outer disk; this is presumably the twist noted by Elmegreen et al. (1996) in a $B$-band atlas image. Because its position angle differs by only $6^{\circ}$ from that of the outer disk, we consider this an inner disk; given the low amplitude of the ellipticity peak, it is the weakest case for an inner disk in our sample. The color map shows a slight inward reddening trend which appears to follow the isophotes; this may be associated with the metallicity gradient.

The WIYN $R$-band ellipse fits also show a strong outer ellipticity peak at $a \sim 45^{\prime \prime}$; unsharp masking with high smoothing $(\sigma=40)$ shows barely perceptible hints of a bar signature at this radius. While it is tempting to suggest that this is a (rather round) bar - and thus that this galaxy is double-barred - the ellipticity peak could instead represent the outer disk, with rounder isophotes outside due to a luminous halo (e.g., Michard \& Marchal 
1993). Lacking a clear indication of a bar, we adopt the outer disk interpretation; deeper images are clearly desirable.

\subsection{NGC 2950: Double bar, nuclear ring}

Figure 16: This is the second most strongly doublebarred galaxy in the sample, with the secondary bar visibly distorting the isophotes. Although Kormendy (1982) called this the "prototypical SB0 galaxy with a triaxial bulge," we agree with W95 that the distortion is in fact a bar.

The ellipse fits yield a PA for the outer disk of $120^{\circ}$, or $25^{\circ}$ less than given by RC3. There is a shoulder in the position angles $\left(\mathrm{PA} \approx 135^{\circ}\right)$ corresponding to the lens, and very clear features due to the primary and secondary bars. Figure 4 shows unsharp masks of this galaxy, highlighting both bars; Figure 5 shows how the PA for the secondary bar derived from the disky isophotes and the unsharp mask differs from that of the ellipse fits. W95 called attention to a weak ellipticity peak between the primary and secondary bars at $a \sim 10^{\prime \prime}$. This peak occurs at a position angle close to that of the outer disk, coincident with a weak $B_{4}$ peak which suggests disky isophotes, so it might indicate a faint inner disk rather than a triaxial bulge.

Unsharp masking of the WFPC2 image (panel f) shows the secondary bar quite clearly; the parallel straight ends of the bar seem to suggest that this bar, at least, has the square-ended isophotes characteristic of large-scale bars in early-type galaxies (as in the outer bar of this galaxy; see also Athanassoula et al. 1990). The unsharp masks also show a faint nuclear ring just outside the inner bar; this ring is smooth and does not appear in any of the color maps. It is a weaker feature than either of the other two stellar nuclear rings (NGC 936 and NGC 3945), and thus rather hard to measure; the values in Table 5 are crude estimates. The orientation and observed ellipticity are consistent with a circular ring in the galaxy plane, though we cannot rule out a moderately elliptical ring; in addition, unsharp masking with low smoothing ( $\sigma=5$, not shown) hints at a possible diamond-shaped, four-armed structure, which argues against a perfectly circular ring. The HST color maps (panel e) show only a smooth inward reddening trend. Unsharp masking does seem to show a couple of faint, very thin dust lanes inside the nuclear ring; the stronger of these matches a slight asymmetry in the color maps (about $2^{\prime \prime}$ north-northeast of the nucleus in panels e and $\mathrm{f})$.

\subsection{NGC 2962: Double bar}

Figure 17: The ellipse fits to the WFPC2 F814W image of this galaxy show a strong ellipticity peak at $a \sim 0.7^{\prime \prime}$, which could be mistaken for a secondary bar. Careful inspection of the unsharp masks (of both WIYN and HST images) reveals that this peak is almost certainly due to strong circumnuclear dust lanes (see panel e). The WIYN and HST isophotes and unsharp masks do reveal the existence of a genuine secondary bar, whose presence in the ellipse fits is marked by the minimum at $a \approx 3.4^{\prime \prime}$, and by a strong positive $B_{4}$ signature peaking at $a \approx 3.6^{\prime \prime}$; we use the mean of these two measurements for the secondary bar's "maximum ellipticity" radius. The secondary bar lies at a position angle of $\approx 90^{\circ}$ - approximately $80^{\circ}$ differ- ent from the best-fitting ellipse! We suspect this difference results from an oblate central bulge - or possibly an inner disk - which dominates the inner light of the galaxy. The isophotes just outside the secondary bar in panel $\mathrm{f}$ are clearly elongated north-south, parallel with the outer disk, as would be expected from such a bulge. Since the distortions introduced by the secondary bar are almost at right angles to the isophotes outside, they force the fitted ellipses to be rounder - and introduce some slight uncertainty into their PA - but do not substantially change their orientation.

The color maps show dust lanes both outside and inside the primary bar. The inner lanes visible in the WIYN images (panel c) are almost all in the south half of the primary bar, which is somewhat odd given that the disk major axis runs north-south. This mightindicate off-plane gas in the central regions, but, lacking any other evidence, we do not consider this galaxy to be a polar ring candidate. (Comparison of the major-axis stellar velocity curve in Simien \& Prugniel 2000 and the Arecibo H I mapping by Burstein et al. 1987 indicates that the outer $\mathrm{H} \mathrm{I}$ and the stars are corotating.) The secondary bar appears to show up in the color map as an asymmetric red feature elongated at $\mathrm{PA} \approx 90^{\circ}$; unsharp masking of the WFPC2 image shows numerous strong dust lanes inside the secondary bar, mostly on the north side, and what may be a very narrow dust ring (semi-major axis $\approx 0.4^{\prime \prime}$, ellipticity $\approx 0.7, \mathrm{PA} \approx 110^{\circ}$.

\subsection{NGC 3032: Nuclear spiral}

Figure 18: It is difficult to identify even one bar in this galaxy. The weak ellipticity peak at $r \approx 8^{\prime \prime}$ appears to be the bar referred to by de Vaucouleurs (1957); in the $B-R$ color map, it is surrounded by a strong dust ring. While this ring might be considered an inner ring surrounding a bar, it is unusually asymmetric for such a ring, and the "bar" may simply be a side effect of dust extinction.

In the NICMOS2 image, the ellipticity variations appear to be due to spiral arms and dust lanes. Regan \& Mulchaey (1999) classified the nuclear regions as "spiral," using a colormap constructed from WFPC2 F606W and NICMOS2 F160W images. Our unsharp mask of the F606W image (panel d) shows that the entire region inside the $B-R$ dust ring is indeed a nuclear spiral, with luminous patches and arms in addition to dust lanes; the brightest regions in our unsharp mask generally match the bluest regions in Regan \& Mulchaey's color map.

Schweizer \& Seitzer (1988) found interleaved "ripples" throughout this galaxy; these are faintly visible in our unsharp masks, suggesting a past merger. The innermost "ripple" ( 8 " W of the nucleus) is blue and may be part of the nuclear spiral. There is no indication of off-plane gas or dust; the H I profile width $W_{50}=161 \mathrm{~km} \mathrm{~s}^{-1}$ (DuPrie \& Schneider 1996) is consistent with the galaxy's low inclination.

\subsection{NGC 3185: Dust-obscured}

Figure 19: This is an actively star-forming galaxy in a Hickson Compact Group (Hickson, Kindl, \& Auman 1989, galaxy 44c), with abundant molecular gas (Leon, Combes, \& Menon 1998).

Unsharp masking shows that the bar of this galaxy 
is surrounded by an inner ring composed of two tightly wrapped spiral arms, coinciding with the ring of $\mathrm{H}$ II regions noted by González Delgado et al. (1997). The color map also shows dust lanes on the inner edges of the ring, leading-edge lanes in the bar (strongest in the SE half of the bar), and a possible dusty nuclear ring. The nuclear regions are asymmetric and quite blue, perhaps indicating star formation; González et al. found extended $\mathrm{H} \alpha$ in the nuclear region.

The ellipse fits show considerable position angle changes within the bar; this is almost certainly due to the strong dust lanes. The innermost ellipticity peak is suggestive of a secondary bar, but the strong nuclear dust lanes make it impossible to be certain without infrared observations.

\subsection{NGC 3412: Inner disk}

Figure 20: The bar manifests itself as a strong position angle twist and a weak ellipticity peak. Much more pronounced is an inner ellipticity peak with the same position angle as the outer disk: one of the stronger inner disks in our sample. (The twisting in position angle between the bar and the inner disk was noted by Shaw et al. 1995 in the IR.) Unsharp masking shows a smooth elliptical feature, with no signs of bar-like structure.

The $B-R$ color map is almost featureless. Fisher, Franx, \& Illingworth (1996) noted a high central H $\beta$ absorption-line strength with a declining outward gradient in their optical spectra, suggestive of a younger stellar population. Though this would seem to agree with the blue nucleus and extended blue structure reported in the near-IR by Shaw et al. (1995), we can find no sign of either in our color map. Instead, there is a suggestion of a red asymmetry in the region of the inner disk, possibly a sign of dust.

The peak in ellipticity at $a \sim 55^{\prime \prime}$ is apparently due to a large lens component (noted by Kormendy 1979); we measure a lower ellipticity of $\approx 0.37$ further out, at the limits of reliable isophote fits, and base the disk inclination estimate on that.

\subsection{NGC 3489: Dust-obscured, nuclear ring}

Figure 21: Although the dust lanes in this galaxy are complex and asymmetric, Bertola et al. (1995) reported similar kinematics for both ionized gas and stars, so the dusty gas is probably in the disk. Dust lanes distort the inner isophotes, making the bar difficult to distinguish; we rely on the isophotes and the unsharp mask to derive its position angle. In the ellipse fits, it shows up most clearly as the sharp twist in position angle between $a \approx 10^{\prime \prime}$ and $20^{\prime \prime}$. Unsharp masking shows that the strong ellipticity peak outside the bar is due to an outer ring (see panel e).

The color map of panel $f$ shows a dusty nuclear ring, with a position angle similar to that of the outer disk. The interior of the nuclear ring is rather blue, smooth and uniform, with no signs of a nuclear spiral or other dust features. The strong dust lanes so close to the nucleus could be masking inner features, but the innermost ellipticity peak appears to be due to the dust lanes of the nuclear ring.

\subsection{IC 676 (Mrk 731): Dust-obscured}

Figure 22: This is a "double-nucleus" Markarian galaxy, with an extremely narrow bar and peculiar dust lanes. Both of the two bright, blue "nuclei" are emission-line sources (Nordgren et al. 1995; Contini, Considere, \& Davoust 1998). The CO spectra of Contini et al. (1997) - $W_{50}=100 \mathrm{~km} \mathrm{~s}^{-1}, W_{20}=128 \mathrm{~km} \mathrm{~s}^{-1}$ - are consistent with the low inclination that we derive from the outer isophotes.

The bar is bordered by red regions in the color map; there is a suggestion of two dust lanes, parallel with the bar. The stellar spiral arms leading off the ends of the bar (best seen in unsharp masks) suggest clockwise rotation, though this would put the straight dust lanes on the trailing edges of the bar. The dust lanes are joined by a narrow lane which crosses the bar between the two blue nuclei. Several other blue regions strung along the bar are symmetrically spaced with respect to the central dust lane. The spiral arms suggest that the upper limit to the bar's radius is closer to $17^{\prime \prime}$ or $18^{\prime \prime}$ than the $a_{\min }$ or $a_{10}$ values from the ellipse fits.

\subsection{NGC 3729: Dust-obscured}

Figure 23: Our WIYN observations of this galaxy were badly affected by bright sky conditions, and we were unable to re-observe it. Only the long $R$-band exposure has usable data, and there is essentially no discernible signal above the sky outside of the inner ring. Consequently, we have used the $B$ and $R$ images from Tully et al. (1996), made available via NED. These were taken with the Mauna Kea $2.24-\mathrm{m}$ telescope, using a $500 \times 500 \mathrm{CCD}$ with $0.598^{\prime \prime}$ pixels. We supplement these with ellipse fits to the central region from the WIYN image, which has higher resolution.

NGC 3729 is believed to be interacting with its "peculiar" neighbor NGC 3718, although Schwarz (1985) found only tenuous evidence for a tidal bridge in $\mathrm{H}$ I between in the two galaxies, and more recent H I observations (see Cox \& Sparke 1996) do not show such a bridge. There is some evidence for distorted optical structure on the NE side of the galaxy (panel a of Figure 23); this blob of excess light appears quite blue in the color map (as noted by Verheijen \& Sancisi 2001), so it may be a region of recent star formation.

The color map shows that the inner ring surrounding the bar is blue; Usui et al. (1998) noted $\mathrm{H} \alpha$ emission from this ring and Verheijen \& Sancisi (2001) indicate that most of the galaxy's H I is in the ring as well. Inside, there are several strong dust lanes. The ellipse fits for $a \lesssim 3^{\prime \prime}$ are distorted by the presence of a bright "second nucleus" about $2.6^{\prime \prime}$ east-northeast of the galaxy center. This feature is partially resolved in the WIYN $R$ image (FWHM more than twice the stellar FWHM), so it is probably not a star. It is noticeably blue in the colormap, so it may be a luminous, young star cluster. The combination of this feature and the strong dust lanes means that we cannot determine whether there are additional structures inside the bar.

Our determination of the outer disk orientation and inclination is based on the outer isophotes, and agrees well with the H I kinematic determinations of Verheijen \& Sancisi (2001), though the latter might be affected by noncircular motions in the inner ring. 


\subsection{NGC 3941: Double bar, off-plane gas}

Figure 24: This galaxy was recently noted for possessing ionized gas near the center which counter-rotates with respect to the stars (Fisher 1994, 1997). In fact, published $\mathrm{H}$ I maps indicate that all the gas in this galaxy is counterrotating. The H I synthesis map (van Driel \& van Woerden 1989) shows that the neutral gas is found primarily in two rings. The outer ring is aligned with the stellar disk and has approximately the same ellipticity. The inner H I ring appears almost circular; if it is intrinsically circular, then it must be inclined $\sim 20^{\circ}$ or more with respect to the stellar disk. Both rings share the same sense of rotation as that of the inner ionized gas observed by Fisher; thus, all the observed gas in this galaxy counter-rotates with respect to the stars. We argue below that some or all of the gas may be off-plane.

Our images show a symmetric stellar disk oriented almost N-S, with a small primary bar. Using the isophotes, we estimate the position angle of this bar to be $\approx 166^{\circ}$; it is probably a typical "rectangular" S0 bar (see Athanassoula et al. 1990), and the extended "corners" produced by projection skew the ellipse fits (Figure 6). Unsharp masking $(\sigma=20$, not shown) indicates weak stellar spiral arms outside the primary bar, with a counter-clockwise trailing orientation; these may be responsible for the ellipticity peak outside the bar in the ellipse fits. Our inclination for the outer disk is based on the outermost isophotes, at $a \sim 120^{\prime \prime}$.

The ellipse fits and unsharp masks also show a secondary bar (see Figure 5). The position angle in the ellipse fits is only $10^{\circ}$ different from our estimate of the outer disk orientation - which would meet our inner disk criteria but the isophotes and unsharp masks indicate a position angle $15^{\circ}$ further to the east, so we consider this a secondary bar. The structure of this feature in the unsharp masks also supports a bar classification.

The primary bar is redder than the outer disk, with a smooth color gradient following the isophotes. This is fairly typical for galaxies in our sample, including those with no evidence for significant dust (e.g., NGC 4665), and suggests that the gradient is due to changing stellar colors.

However, within the bar there are clear indications of spiral dust lanes with a peculiar orientation (panels d and f). While they have the same sense of rotation - trailing counter-clockwise - as the faint stellar arms outside the bar, the fact that the gas in this region is counterrotating presents us with an interesting problem. Either some of the spirals in this galaxy are leading, or else the gas and stars are not coplanar. The dust lanes in the bulge region are almost entirely confined to the east side of the galaxy, so they could be part of an inclined gas disk, as in NGC 2655, NGC 2787, and NGC 4203. The minor-axis spectrum of Fisher (1997) shows rotation in the ionized gas, further indication that the gas and stars are misaligned. We suggest that the inner ionized gas and outer H I are all on inclined orbits, almost perpendicular to the stellar disk. In this case, the eastern sides of the gas disks/rings are closer to us, while the nearer side of the stellar disk is to the west, and both stellar and dusty spirals are trailing. Since the inner gas is probably offplane, we do not class this as a "nuclear spiral," though the central dust lanes are partly spiral in shape.

\subsection{NGC 3945: Double bar, nuclear ring, inner disk}

This excessively complex galaxy — with two bars, an inner disk, and a stellar nuclear ring - was discussed in detail by Erwin \& Sparke (1999). The measurements in Table 5 are the same, except for the position angles of the two bars and the ellipticity of the nuclear ring. Examination of unsharp masks and of the isophotes indicates the position angles for both bars that are different from those given by the ellipse fits. We also measured a semiminor axis value for the nuclear ring of $\approx 2.8^{\prime \prime}$, using the $\sigma=20$ unsharp mask of the WFPC2 F814W image; this gives an ellipticity of 0.57 for the nuclear ring (the value in Erwin \& Sparke was from the ellipse fits). This suggests that the nuclear ring is intrinsically elliptical, as long as the galaxy's inclination is $<67^{\circ}$.

\subsection{NGC 4045: Dust-obscured}

Figure 25: This galaxy is a splendid example of a dusty, star-forming Sa, similar in some respects to NGC 2273. The outer disk has two blue spiral arms which wrap almost $180^{\circ}$ around and appear to dimple in at the bar ends, forming an $\mathrm{R}_{1}^{\prime}$ outer ring; this causes the ellipticity peak at $a \approx 66^{\prime \prime}$. Numerous patchy blue spiral arms form a partial inner pseudo-ring around the bar. These arms are almost certainly sites of recent or ongoing star formation; Usui et al. (1998) traced the "inner ring" in $\mathrm{H} \alpha$ emission. The dust lanes are stronger on the $\mathrm{S}$ side, suggesting that side of the galaxy is nearer.

The bar itself lies almost north-south; unsharp masking and the color maps show the characteristic leading-edge dust lanes, along with the associated "spur" dust lanes, as in NGC 2273. The two leading-edge dust lanes converge on what might be a nuclear dust ring, with some bright asymmetries near the nucleus, though this is at the limits of our resolution.

\subsection{NGC 4143: Nuclear spiral, inner disk}

Figure 26: Unsharp masking indicates that the bar in this galaxy lies at a $\mathrm{PA}$ of $\approx 165^{\circ}$, rather than the $156^{\circ}$ suggested by the ellipse fits. In the ellipse fits, the bar shows up as a pronounced position angle twist, but not as a distinct ellipticity peak. We identify $a_{\max }$ with the maximum in position angle; $a_{\min }$ cannot be defined. Further in, the ellipse fits show an ellipticity peak at a position angle only $2^{\circ}$ different from that of the outer disk. This is then a clear, if not particularly strong, inner disk candidate.

The $B-R$ color maps shows one or two possible dust lanes outside the bar, and several strong dust lanes in the nuclear region. The nuclear lanes are complex and asymmetric, but appear to form a spiral pattern, trailing clockwise, particularly in unsharp masking of the WFPC2 image; Martini et al. (2001) noted this spiral pattern as well, classifying it as "flocculent." (By contrast, a weak counter-clockwise trailing stellar spiral can be seen in unsharp masks, outside the bar - this might indicate that the inner gas is not corotating with the stars.) A pair of dust lanes south of the nucleus blur into a single lane in the $B-R$ color map; these have a different orientation than the spiral lanes. In the F606W-F160W color map, the strongest dust patches are red, while the nucleus itself is blue (probably an AGN - Ho et al. 1997). 


\subsection{NGC 4203: Off-plane gas}

Figure 27: This weakly barred galaxy harbors one of the best-documented AGNs in our sample, with broad $\mathrm{H} \alpha$ emission, a power-law X-ray spectrum, and a compact UV pointlike nucleus (Ho et al. 1997; Iyomoto et al. 1998; Barth et al. 1998). Westerbork H I observations by van Driel et al. (1988) show two H I rings, a nearly circular inner ring with $r \approx 44^{\prime \prime}$, probably coplanar with the stellar disk, and an extended, elliptical outer ring beyond the optical disk; the latter is probably tilted (by $34^{\circ}$ or $87^{\circ}$ ) with respect to the optical disk.

The bar is a weak, elliptical distortion aligned with the outer disk; this alignment means we cannot define $a_{10}$. Our value for $a_{\min }$ is much larger than $a_{\max }$; unsharp masking suggests the true end of the bar is probably just outside $a_{\max }$.

The $B-R$ color maps show counter-clockwise spiral dust lanes outside the bar. Inside the bar, the dust is much stronger, and confined to the region east of the blue nucleus. The WFPC2 unsharp masks and color maps show concentric arcs of dust, centered on the nucleus; this suggests an inclined gas disk, extending above the stellar disk on the east side. Together with the evidence for misalignment between the outer $\mathrm{H}$ I and the optical disk, this leads us to put the galaxy in our off-plane gas class (it is listed as "Kinematically Related" to polar rings in the catalog of Whitmore et al. 1990).

Because the dust lanes are quite small-scale and onesided, we have a relatively unobstructed view of the central regions, so we do not consider this a dust-obscured galaxy. Neither the ellipse fits nor the unsharp masks show any evidence for an inner bar; an inner disk would be extremely hard to detect, since the galaxy is close to face-on.

\subsection{NGC 4245: Nuclear spiral, nuclear ring}

Figure 28: This galaxy is part of a group within the Coma I cloud which also includes NGC 4310 and NGC 4314; we adopt a distance of $12 \mathrm{Mpc}$ for all three galaxies, based on the distances for galaxies in that cloud given by Forbes (1996). García-Barreto, Downes, \& Huchtmeier (1994) have suggested that this group may be analogous to an extremely poor cluster, especially since the galaxies in the central part of the group (projected $r<130 \mathrm{kpc}$, which includes all three of the galaxies in our sample) are all deficient in H I. In fact, NGC 4245 was one of the three most $\mathrm{H}$ I-deficient spirals in the group (the most deficient being NGC 4314), though Gerin \& Casoli (1994) found the molecular gas content of all three to be typical for their Hubble types.

Like its better-studied sibling NGC 4314, NGC 4245 harbors a star-forming nuclear ring. Leading-edge dust lanes that appear to flow into the ring are visible in both the unsharp mask and the color maps. The nuclear ring shows up in the ellipse fits as a rapid change in ellipticity and a discontinuity in position angle; we measure the ring's size, shape, and orientation directly from the WFPC2 image. In the unsharp mask of that image, we can see that the ring is made up of bright knots mixed with spiral dust lanes; the latter lead into a nuclear spiral which appears to continue all the way into the nucleus. The $B-R$ color map (panel f) shows that the brightest regions of the nuclear ring are blue, consistent with their being sites of recent or ongoing star formation.

We find no evidence for an inner bar within the nuclear ring in the WFPC2 unsharp mask; the variations in the ellipse fits are almost certainly due to the dust lanes of the nuclear spiral. These dust lanes are weak enough that feel fairly confident that there is no inner bar in this galaxy, despite not having near-IR images.

\subsection{NGC 4310: Dust-obscured}

Figure 29: This highly inclined galaxy is a member of the same group as NGC 4245 and NGC 4314 (see discussion of NGC 4245, above). The color maps and unsharp masks show strong dust lanes on the western side of the disk, along with blue regions closer to the center. Our identification of the (sole) bar is rather uncertain, and any central structures could be hidden by the complex of dust lanes.

\subsection{NGC 4314: Double bar, nuclear ring, nuclear spiral}

Figure 30: This galaxy has both a well-studied nuclear ring and a secondary bar; the availability of both WFPC2 and NICMOS images, and the large size of the secondary bar, make it idea for studying the shape of dust lanes within a secondary bar. See the notes for NGC 4245 and NGC 4310 (above) for a discussion of this galaxy's environment, which may be responsible for its extreme $\mathrm{H} \mathrm{I}$ deficiency (García-Barreto et al. 1994).

As mentioned in Section 4.3, defining the length of this galaxy's primary bar is problematic. Published measurements agree on lengths of $60-75^{\prime \prime}$. The DSS and WFPC2 mosaic images (panels a and c) show that the classic, narrow bar, with notably square ends (Athanassoula et al. 1990), comes to an end at approximately this length (roughly at $a_{\max }=70^{\prime \prime}$ ). However, the isophotes remain elongated and parallel with the bar well beyond that radius, as can be seen in both the images and the ellipse fits, as well as in the near-IR images of Quillen et al. (1994) and the ellipse fits of Friedli et al. (1996). Consequently, $a_{10}$ and $a_{\text {min }}$ give much larger values for the bar size (113" and $127^{\prime \prime}$, respectively). It is not clear why the galaxy has this appearance; Quillen et al. and Patsis, Athanassoula, \& Quillen (1997) modeled the bar and placed corotation at $r=70^{\prime \prime} \pm 10^{\prime \prime}$. If that is the case, we would not expect the stars to have a distribution parallel to the bar beyond that radius. One possibility is that we are seeing the bright inner parts of the two spiral arms which cross over, or trail off from, the bar ends, forming what may be an incomplete outer ring. The WFPC2 mosaic image suggests that these arms extend slightly past the bar ends on the leading side, so that the isophotes remain roughly parallel to the bar even though they are no longer bar-shaped. Nonetheless, it is curious that the combined stellar distribution seems parallel to the bar well beyond the bar end itself, particularly if corotation is close to the bar end.

Benedict et al. (1996) presented CO imaging of the bar. Using this and earlier CO data from Combes et al. (1992), they constructed a rotation curve and identified orbital resonances for the primary bar, assuming that it ended shortly before corotation. This rotation curve produced two inner Lindblad resonances, with the nuclear ring lying between them and the inner bar ending close to the inner ILR of the primary bar. 
The nuclear ring is visible in the HST images as both a set of bright, blue knots and several curved dust lanes, and has recently been studied in detail by Benedict et al. (2002), who find cluster ages of 1-5 Myr. At least some of the star formation sites show up as bright knots in the F160W unsharp mask (panel e), which suggests that some of the near-IR light in the ring is from red supergiants. Our measurement of the ring's size and orientation are based on the blue features in the WFPC2 color map (panel f). The position angle we measure $\left(135^{\circ}\right)$ is very close to that determined by Benedict et al. $(2002)\left(137^{\circ}\right)$ from an ellipse fit to the distribution of blue star clusters, though they derived a slightly larger semi-major axis $\left(7.4^{\prime \prime}\right.$ versus our $\left.6.8^{\prime \prime}\right)$. As reported by Combes et al. (1992) and Benedict et al. (1996), this ring is slightly outside the main CO ring, but is roughly coincident with the ring of $\mathrm{H} \alpha$ emission (González Delgado et al. 1997) and $6 \mathrm{~cm}$ radio continuum emission (García-Barreto et al. 1991(@); it also matches the HST $\mathrm{H} \alpha+[\mathrm{N} \mathrm{II}]$ map of Benedict et al. (2002). The strongest dust lanes in the unsharp mask and the color map do seem to lie just inside the blue ring of star formation, consistent with the idea that the traces the molecular gas.

Also clearly visible are two blue arms outside, trailing away from the NW and SE ends of the ring, which resemble features seen outside the nuclear ring in NGC 2273 (above). These make up the elliptical blue region reported by Benedict et al. (1993). Benedict et al. (2002) pointed out that these arms are oriented almost perpendicular to the nuclear ring and to the outer bar, and are located in between the two ILRs suggested by the CO-derived resonance curves of Benedict et al. (1996); they suggested that these arms might be associated with $x_{2}$ orbits of the outer bar. They also found colors consistent with ages of $\sim 100$ $200 \mathrm{Myr}$ for the arms, indicating a previous episode of star formation, perhaps associated with the outer of the two ILRs.

The inner bar was first noticed by Benedict et al. (1993), using WF/PC1 images in the $I$ band. It is even more prominent in the NICMOS2 F160W image (e.g., the ellipse fits in panel $\mathrm{b}$ and the unsharp mask in panel e; also see the bulge-subtracted image in Ann 2001), with an ellipticity peak just inside the nuclear ring and a position angle of $136^{\circ}$ - parallel with the nuclear ring (as noted by Ann 2001), close to the position angle of the outer bar, but much different from that of the outer disk. (The ellipticity peak outside the ring is due to the blue spiral arms noted above.) The presence of this bar has been confirmed by both Ann (2001) and Benedict et al. (2002), using the NICMOS and WFPC2 images, respectively. In contrast to Benedict et al., however, we argue that the inner bar is probably not parallel with the outer bar. The use of the F160W image (less affected by dust) provides a better estimate of the inner bar's orientation; in addition, there is little uncertainty in the position angle of the outer bar (position angles $<145^{\circ}$ are clearly ruled out). This distinction is important because an inner bar parallel to the outer bar can be explained as the result of $x_{1}$ orbits inside a double ILR in a single bar. Our measurements suggest that the inner bar actually trails the outer bar by $\approx 10^{\circ}$, and is thus likely to be independently rotating. In support of this scenario, Ann (2001), who also noted the $\approx 10^{\circ}$ mis- alignment between outer bar and nuclear ring, found that agreement between gas flow in hydrodynamic simulations and the visible dust lanes of this galaxy was better when the inner bar was independently rotating.

The PC2 unsharp mask and the color map show a wealth of dust lanes within the nuclear ring. The lanes are aligned with the secondary bar, and rotationally symmetric about the center. Two of the lanes are clear analogues of the leading-edge lanes in the primary bar, with the same sense of rotation. Curiously, they appear to extend past the midpoint of the inner bar, though there does not appear to be a dust ring within the inner bar. For simplicity, we classify these lanes as a "nuclear spiral."

\subsection{NGC 4386: Inner disk}

Figure 31: The bar in this galaxy is almost aligned with the outer disk, and appears primarily as a peak in the ellipticity, though it also shows up as a modest red feature in the $B-R$ color map. Inside, there is a weak secondary ellipticity peak aligned with the outer disk. This feature, which fits our criteria for an inner disk, appears as a striking red zone in the color maps, reddest at the center (a change of $\approx 0.22$ in $B-R$ ).

Unsharp masking shows two peculiar features: a faint symmetric brightening just outside the inner disk, which might indicate a faint stellar nuclear ring (panel d); and a hint of bar-like structure in the inner disk itself (panel f). Our resolution is not good enough for us to decide if this is really a bar; nonetheless, NGC 4386 is a good candidate for an inner disk which is actually a secondary bar.

\subsection{NGC 4643: Nuclear spiral, inner disk, off-plane gas?}

Figure 32: This is a strongly barred galaxy with an unusually thin bar; it had the narrowest bar of the six early-type barred galaxies studied by Ohta et al. (1990). The stellar velocity field of Bettoni \& Galletta (1997) suggested circular rotation in the disk (apart from bar-related deviations), with $i \approx 40^{\circ}$ and a line-of-nodes at $\approx 50^{\circ}$ (see also Magrelli, Bettoni, \& Galletta 1992); this agrees well with our values for the outer disk isophotes. Whitmore et al. (1990) classed this as an "Object Possibly Related to Polar Rings," noting that an unpublished image showed "a very faint, disklike (i.e., about E8) feature ... aligned along the major axis extending to about three times the optical radius of the inner galaxy." A similar feature has been reported by David Malin (1998, private communication). No sign of this appears in our images, though this may simply be because of the relatively short exposure.

This galaxy was one of the three strongest double-bar candidates found by Shaw et al. (1995), due to strong position-angle twists within the bar. Our ellipse fits show that the twist is due to an inner elliptical component at the same position angle as the outer disk: we interpret this feature as an inner disk. In a $\sigma=2$ unsharp mask, the inner disk actually shows some signs of being a ring, but the rather low resolution of our images ( $R$-band seeing $=1.3^{\prime \prime}$ ) means we cannot be certain.

The $B-R$ color maps show tightly wrapped spiral arms in a fairly narrow zone just outside the bar; the mix of blue and red arms suggests both dust and recent star formation. In the vicinity of the inner disk we see a dusty nuclear spi- 
ral, with the same sense of rotation as the spiral arms outside the bar. A separate dust lane, slightly curved, to the NW of the nucleus could be part of a polar dust lane; the orientation is roughly the same as the faint optical extensions reported by Whitmore et al. (1990). However, the H I width of $W_{20}=351 \mathrm{~km} \mathrm{~s}^{-1}$ reported by Wardle \& Knapp (1986) is consistent with Bettoni \& Galletta's observed stellar $V_{\max }$ of $140 \mathrm{~km} \mathrm{~s}^{-1}$ (at $r=15^{\prime \prime}$ ), so there is no evidence here for off-plane gas.

\subsection{9. $N G C 4665$}

Figure 33: This galaxy is a relatively featureless SB0, with a strong bar and only a few asymmetries in the outer disk and a curious twist inside the bar to mar an otherwise canonically simple appearance. Ohta et al. (1990) performed an extensive analysis of the surface photometry and decomposed the galaxy into disk, bulge, and bar components. It had the highest bar-to-disk luminosity ratio of the six galaxies in their sample: almost half the light between the bulge and the ends of the bar was in the extracted bar model.

The ellipse fits show the bar, but no clear signs of any other structures. There is a very weak increase in ellipticity near the very center $\left(a \lesssim 1.5^{\prime \prime}\right)$; due to the poor seeing $\left(1.2^{\prime \prime}\right)$ we cannot determine what, if anything, this is due to. We do see the twist in PA along the bar noted by Jungwiert et al. (1997) in their near-IR images - amounting to $\approx 12$ degrees between $a=45^{\prime \prime}$ and $a=1^{\prime \prime}$. This could be a sign of mild triaxiality in the bulge.

Weak stellar spiral arms appear to trail off the ends of the bar, with the southern arm somewhat stronger than the northern one. These arms can also be seen in the processed images of Ohta et al. (1990), where axisymmetric components of the galaxy have been modeled and subtracted. Both arms are red features in the color map, which may indicate some dust content. More striking is the outer, red arc in the E side of the galaxy. Its position and shape are suggestive of a dusty outer ring, although there is no counterpart in the $\mathrm{W}$ side of the disk. This color asymmetry is interesting in light of the fact that this was one of the six least asymmetric galaxies in the 60galaxy $R$-band sample of Rudnick \& Rix (1998), based on $R$-band images.

Neither the color map nor unsharp masks show any evidence for dust lanes or rings in the inner regions. A general inward reddening trend is visible - the galaxy center is $\sim 0.4$ magnitudes redder than the disk just outside the bar - and the isochromes twist to follow the isophotes. This suggests that the isophote twist is not due to leading-edge dust lanes, which would produce a twist in the opposite sense. Unlike the isophotes, however, the isochromes remain elongated into the galaxy center.

\subsection{NGC 4691: Dust-obscured}

Figure 34: This is an unusual and complicated galaxy, which bears some resemblance to IC 676 . Like that galaxy, it has an extremely narrow bar with several distinct blue regions of recent star formation and a dust lane crossing straight through (or over) the bar; but it lacks the other galaxy's symmetry.

The H I spectra of Huchtmeier \& Seiradakis (1985) and Richter \& Huchtmeier (1987) are somewhat peculiar and possibly asymmetric, and the velocity widths reported by Huchtmeier \& Seiradakis $\left(W_{20}=167 \mathrm{~km} \mathrm{~s}^{-1}\right.$ and $W_{50}=76 \mathrm{~km} \mathrm{~s}^{-1}$ ) are not consistent with ordered, circular rotation. Richter \& Huchtmeier give a smaller value of $W_{20}=132 \mathrm{~km} \mathrm{~s}^{-1}$, which is only barely consistent with the apparent inclination (for $i=42$, it implies $V_{\text {circ }} \approx 100$ $\mathrm{km} \mathrm{s}^{-1}$ ). Young et al. (1995) reported an extremely narrow $\mathrm{CO}$ width $\left(W_{50}=35 \mathrm{~km} \mathrm{~s}^{-1}\right)$, and Wiklind, Henkel, \& Sage's (1993) CO velocity map for $r \lesssim 20^{\prime \prime}$ showed essentially no velocity gradient; they argued that the galaxy was face-on. All of this suggests that the gas in this galaxy is either not coplanar with the stars, not in ordered motion, or both.

The outer disk appears somewhat distorted. The bar itself ends in two stubby spiral arms which partly wrap around it to form a partial inner ring; inspection of unsharp masks suggests that the bar may actually end at $a \sim 42^{\prime \prime}$, with the beginnings of the inner ring keeping the isophotes approximately parallel until $a_{10}=55^{\prime \prime}$. Because of the complicated structure within the bar, the ellipse fits are unreliable for $a \lesssim 25^{\prime \prime}$, and the fitting algorithm fails altogether for $\left.a<3 . .^{\prime \prime}\right)$.

The color maps show a total of seven distinct blue regions in the bar, four of which correspond to the $\mathrm{H} \alpha$ bright knots studied photometrically and spectroscopically by García-Barreto et al. (1995); the latter are the four brightest "nuclei" in the $R$-band image (panel e). The easternmost blue region is separated from the rest by a narrow dust lane, which appears to connect with strong dust lanes which run along the north and south sides of the bar. There is some resemblance to the bar-crossing lane in IC 676 , but in this galaxy none of the structure is symmetric about the bar.

\subsection{NGC 5338: Dust-obscured}

Figure 35: This galaxy, among the closest in our sample $(D=11.5 \mathrm{Mpc})$, shows evidence for dust lanes along middle of the bar. In the center, there is a rather abrupt transition to an asymmetric blue region, possibly connecting with a blue spiral arm to the SE. Although this appears as a change in PA and ellipticity in the ellipse fits, the unsharp masks show no sign of a secondary bar or other regular structures. The innermost regions are complex and asymmetric, possibly due to star formation.

Although the shape of the outer disk argues for a high inclination $\left(i=68^{\circ}\right)$, the $\mathrm{H} \mathrm{I} W_{20}$ is only $87 \mathrm{~km} \mathrm{~s}^{-1}$ (LEDA; $91 \mathrm{~km} \mathrm{~s}^{-1}$ in Wardle \& Knapp 1986). This might indicate that the H I is not coplanar with the stars.

\subsection{NGC 5377: Nuclear spiral, nuclear ring}

Figure 36: This is another galaxy where the outer isophotes are dominated by an outer ring, this time a notably blue one. It seem clear that the galaxy is highly inclined - but how highly inclined is difficult to tell. If we require $V_{\text {circ }}<500 \mathrm{~km} \mathrm{~s}^{-1}$, then $i>21^{\circ}$ (given $W_{20}=380$ $\mathrm{km} \mathrm{s}^{-1}$, Balkowski \& Chamaraux 1983). Misalignment between the outer and inner rings (and the bar) suggests that outer ring may be intrinsically perpendicular to the bar, and that the line-of-nodes lies somewhere between the apparent position angles of the two rings. Unfortunately, this makes determining the inclination more difficult. An intrinsically circular outer ring would imply $i=58^{\circ}$; an 
intrinsically circular inner ring means $i=50^{\circ}$. We adopt $i=55^{\circ}$ and $35^{\circ}$ for the position angle of the line-of-nodes; this is reasonably close to the outer kinematic inclination $\left(\approx 50^{\circ}\right)$ and major axis $\left(\approx 30^{\circ}\right)$ derived from VLA H I observations (Kornreich et al. 2001).

Both the outer and inner rings are quite blue in the $B-R$ color maps, and there is evidence for dust on both the inner and outer edges of the inner ring. There are also strong leading-edge dust lanes within the bar, apparently feeding into a nuclear ring. Determining the size of the bar is difficult, due to the effect of the inner ring on the ellipse fits. We note that the semi-major axis of the inner ring (measured on the color maps) is less than our value of $a_{\min }\left(78^{\prime \prime}\right)$, so the true length of the bar is probably somewhere between $58^{\prime \prime}$ and $70^{\prime \prime}$.

The HST images reveal a dramatic and complex nuclear ring, first described by Carollo, Stiavelli, \& Mack (1998). Examination of unsharp masks and color maps indicates that the ring consists of three parts. The outermost is a red ring of tightly wrapped dust lanes, into which the leading-edge lanes of the bar flow; this is marked in the ellipse fits by an abrupt change in position angle ("NR(R)" annotation in the figure and in Table 5). The next major feature is a bright ring, especially visible in the NICMOS2 unsharp mask (panel f); this was also noted by Carollo et al. It appears in the ellipse fits as an ellipticity maximum, and in both the $B-R$ and HST color maps as a blue region on the $\mathrm{SE}$ side, which suggests that it may be made of younger stars. Finally, there appears to another dust ring right around the nucleus $\left(a \approx 0.85^{\prime \prime}, \mathrm{PA} \sim 30^{\circ}\right)$, best seen in the $V-H$ color map (panel e). Both the outer and inner dust rings show up in ellipse fits to the F606W image (not shown) as discontinuities in the position angle. The nucleus itself appears quite blue. In addition, there are spiral dust lanes in between the two dust rings, threading through the luminous blue ring.

\subsection{NGC 5701: Nuclear spiral}

Figure 37: This galaxy is surrounded by a large, distinct outer ring, elongated roughly perpendicular to the bar (the size listed in Table 5 is more of an upper limit on the ring's size, since the arms making up the ring wind outward over a large range in radius). This coincides with the $\mathrm{H} \alpha$ ring seen by Pogge \& Eskridge (1993); the blue spiral arms visible in the color map are thus almost certainly the result of recent star formation. The optical galaxy lies within a huge, somewhat lopsided H I disk, extending to $r \approx 10^{\prime}$ (DuPrie \& Schneider 1996; Kornreich et al. 2000). The Duprie et al. Arecibo velocity map indicates an approximate PA for the disk line-of-nodes $\sim 45^{\circ}$; Kornreich et al., using VLA data, find $48^{\circ}$. The low velocity width $\left(W_{50}=121 \mathrm{~km} \mathrm{~s}^{-1}\right.$ from Duprie et al. and $139 \mathrm{~km} \mathrm{~s}^{-1}$ from Kornreich et al.) suggests that the H I disk is nearly face-on.

The presence of a detached outer ring means that determining the inclination is difficult. Assuming that the $\mathrm{H} \mathrm{I}$ is coplanar with the stars, and that $100 \mathrm{~km} \mathrm{~s}^{-1}<V_{\text {circ }}<500$ $\mathrm{km} \mathrm{s}^{-1}$, then $7^{\circ}<i<37^{\circ}$. A more stringent upper limit can be found by assuming that the isophotes between the bar and the outer ring, which appear parallel to the bar with $e \approx 0.21$, are those of a lens, and thus intrinsically parallel with the bar. An extremely elliptical inner ring with axis ratio 0.7 (e.g., NGC 1433, slightly beyond the $3-\sigma$ limit in Buta (1995)) would imply $i=28^{\circ}$, which can be taken as a reasonable upper limit. We adopt an intermediate value of $20^{\circ}$; this implies that the outer ring is intrinsically perpendicular to the bar, but is fairly round (axis ratio $=0.85$ ), and that $V_{\text {circ }} \approx 175 \mathrm{~km} \mathrm{~s}^{-1}$.

The bar itself is almost completely dust-free. There is a gradual twist in position angle within the bar, noted by Jungwiert et al. (1997) in their near-IR images. As they point out, the low inclination of the galaxy makes it unlikely that this is a projection effect. Unsharp masking shows a faint nuclear spiral extending out to $r \sim 10^{\prime \prime}$; this may be responsible for the position angle and ellipticity variations for $a<10^{\prime \prime}$. There is no sign of an inner bar or disk, though the low inclination of the galaxy means an inner disk would be quite difficult to detect.

\subsection{NGC 5750: Dust-obscured}

Figure 38: We have found no significant observations of this dusty SB0/a galaxy in the literature, aside from an H I spectrum in Tifft \& Cocke (1988), which gives a velocity width $W_{20}=411 \mathrm{~km} \mathrm{~s}^{-1}$. This seems consistent with the galaxy's rather high inclination, so the $\mathrm{H} \mathrm{I}$ is probably coplanar with the stars. Our $B-R$ color map shows a striking blue inner ring surrounding the bar, with weak evidence for a red ring further out. The region inside the inner ring is quite dusty, though virtually all of the dust lanes seem confined to the northeast side of the galaxy. The two ellipticity peaks in the ellipse fits are almost certainly due to the dust; we see no signs of a secondary bar or inner disk in the unsharp masks, but IR observations are clearly needed to be certain.

\subsection{NGC 6654: Double bar}

Figure 40: There appear to be almost no observations of this galaxy in the literature, aside from photographic surface photometry discussed in Kormendy (1977a,b). No H I or CO emission has been detected (Wardle \& Knapp 1986; Knapp \& Rupen 1996). The $B-R$ color map shows a lacy pattern of tightly wrapped blue spirals in the outer disk. These arms are luminous structures in both the $B$ and $R$ images, so they are probably sites of recent or ongoing star formation; they appear to lie just outside the "plateau" region of Kormendy's surface brightness profiles. There are no significant color variations in the bar itself. Inside the primary bar is a secondary bar; Figure 5 shows that this is one of several inner bars where the ellipse fits give a misleading position angle. There is a suggestion of a color asymmetry in the WFPC2 color map, but it is rather weak. Unsharp masking shows no signs of distinct dust lanes in or around the inner bar.

\subsection{UGC 11920: Dust-obscured}

Figure 39: We have found no previous observations of this galaxy in the literature, aside from an $\mathrm{H}$ I spectrum in Kamphuis, Sijbring, \& van Albada (1996). The spectrum appears to have $W_{50} \sim 300-350 \mathrm{~km} \mathrm{~s}^{-1}$, which is consistent with the galaxy's inclination.

The profusion of foreground stars in the image makes measuring the outer disk's shape and orientation difficult. The bar is almost parallel with the apparent major axis of the disk, so we cannot define an $a_{10}$ length. Within 
the bar, the color map shows a strong, curving dust lane which leads into the central regions; it may be merging with a dusty nuclear ring, but our resolution is not good enough to tell. Interior to this, both the ellipse fits and the unsharp masks suggest a secondary bar - but this may simply be the result of dust lanes. Although we provide measurements for this possible secondary bar, we do not count UGC 11920 among the confirmed double-barred galaxies of our sample.

\subsection{NGC 7280: Double bar, off-plane gas}

Figure 41: This galaxy is similar to NGC 3941: it is double-barred, and there is good evidence for off-plane gas in the nuclear regions.

Two H I studies suggest the possibility that this galaxy is interacting with its neighbor UGCA 429 , a dwarf irregular $4.2^{\prime} \mathrm{ENE}$ of this galaxy with a redshift difference of only $57 \mathrm{~km} \mathrm{~s}^{-1}$. van Driel \& van Woerden (1991) found a weak central H I concentration in NGC 7280, and evidence for a weak extension in the direction of the dwarf irregular; DuPrie \& Schneider (1996), on the other hand, found that the H I in UGCA 429 appeared to extend in the opposite direction from NGC 7280.

Our WIYN images show an elliptical disk with a weak bar almost aligned with the outer disk; unsharp masking shows an apparent outer ring at $r \sim 40^{\prime \prime}$. More striking is the color map, which shows several dust lanes, all in the northern half of the disk. In addition to a long, linear dust lane $\approx 23^{\prime \prime}$ ENE of the galaxy center, there are several short lanes in the central regions, including one arcing right around the nucleus. The combined F606W WFPC2 image shows that the last lane is actually composed of two strong, nearly straight lanes, and a fainter, more curved lane to the west, crossing right over the nuclear regions (panel f). Carollo et al. (1997) suggested that these lanes were "likely rings wrapping several times around the nucleus and perpendicular to it, reminiscent of the famous polar-ring galaxy NGC 2685." We concur; the asymmetric dust distribution further out, and the indirect H I evidence for interaction with UGCA 429, are further evidence for this being a polar ring galaxy. The recent two-dimensional spectroscopy of Afanasiev \& Sil'chenko (2000) confirms this: ionized gas in the inner $10^{\prime \prime}$ rotates orthogonally to the stars.

Although Afanasiev \& Sil'chenko suggest that the innermost elliptical feature in the isophotes, mostly clearly seen in the NICMOS image, is an inclined stellar disk, we disagree. Unsharp masks of both the WFPC2 and the NICMOS images show the characteristic signs of an inner bar; the position angle of this bar, based on measurements of the isophotes and the unsharp masks, is $\approx 115^{\circ}$ (almost 15 degrees away from the position angle given by the ellipse fits). Since the region Afanasiev \& Sil'chenko study includes the transition from an outer bar to an inner bar, their assumption of circular rotation is probably not justified; they also use position angles of $\approx 80--100^{\circ}$, rather different from what we find. Thus, we do not feel that their kinematics necessarily imply an inclined disk.

\subsection{NGC 7743: Nuclear spiral}

Figure 42: This is a fairly well-studied Seyfert galaxy with a dusty nuclear spiral and evidence for star formation in the nuclear regions (e.g., $\mathrm{H} \alpha$ observations by Pogge \& Eskridge 1987; extended UV emission in Barth et al. 1998; near-IR spectrum in Larkin et al. 1998).

The outer isophotes are asymmetric, and there are projections to the NE and SW, especially noticeable at about $85^{\prime \prime}$ from the center; these can also be seen in the images of Kennicut \& Edgar (1986). The nature of the galaxy's H I gas is also confusing: while both Wardle \& Knapp (1986) and Balkowski \& Chamaraux (1983) reported $W_{20}=310$ $\mathrm{km} \mathrm{s}^{-1}$ and Wardle \& Knapp give $W_{50}=258 \mathrm{~km} \mathrm{~s}^{-1}, \mathrm{Lu}$ et al. (1993) report $W_{50}=75 \mathrm{~km} \mathrm{~s}^{-1}$; DuPrie \& Schneider (1996) failed to detect any H I and suggested that previous reports were confused by two $\mathrm{H}$ I clouds to the north (whose redshifts differ from that of the galaxy, however).

The bar is surrounded by an inner pseudo-ring which does not quite close, and which is responsible for both the peak in PA at $a \approx 50^{\prime \prime}$ and the corresponding peak in ellipticity. We identify the bar itself with the minimum in PA at $a \approx 31^{\prime \prime}$; the twisting exterior to the is due to the spiral arms. Both $a_{10}$ and $a_{\text {min }}$ are affected by the spiral arms and are probably overestimates for the bar length.

Within the bar are two parallel dust lanes in the western half, the southernmost of which could be a leading-edge lane; however, no strong lanes are visible in the east half. W95 suggested that this galaxy had a triaxial bulge, on the basis of twisting position angles between 3 and $10^{\prime \prime}$. Since this is the region dominated by complex, asymmetric dust lanes in the color map (see below), we suspect most of the twisting is due to dust, not intrinsic triaxiality.

The dust lanes in the central regions $\left(r \lesssim 8^{\prime \prime}\right)$ are asymmetric and off-center, and do not form an obvious nuclear ring; the interior is rather blue. Unsharp masking of the WFPC2 image (panel e of the figure) resolves this interior into nuclear spiral dust lanes, some of which can be seen in the PC2-NICMOS color image of Regan \& Mulchaey (1999). Although those authors suggested that there was a secondary bar present, on the basis of what appeared to be straight, leading-edge dust lanes, our inspection of the HST images, both WFPC2 and NICMOS, shows no sign of a bar or inclined disk. The ellipticity peak at $a \approx 0.6^{\prime \prime}$ in the NICMOS image is probably due to strong dust lanes near the center (which can be seen in the figures of Regan \& Mulchaey). In particular, there is no hint of any elongated structure with a length appropriate to the "straight" dust lanes Regan \& Mulchaey identify. The unsharp mask of the WFPC2 image (panel e), which is larger in scale and higher in resolution than Regan \& Mulchaey's color map, indicates that the dust lanes they identified are simply parts of an overall nuclear spiral. We conclude that there is probably no secondary bar in this galaxy.

We are indebted to those people who obtained images for us during queue time or their own or shared observing runs: Eric Wilcots, Jay Gallagher, and especially Ted von Hippel, who in addition gave valuable advice and assistance on this project's first observing run. Jay Gallagher provided numerous useful and insightful comments on various drafts of this paper, the thesis it derives from, and the early, exploratory versions of the sample. Witold Maciejewski helped by maintaining a proper theorist's skepticism of observations; he also produced images of model double-bar galaxies and helped provide some of the theo- 
retical motivations for measurement techniques and analysis. We also thank Daniel Friedli and Marc Balcells for helpful comments and criticisms on various drafts of this work. The work reported here forms part of the Ph.D. thesis of Peter Erwin at the University of Wisconsin-Madison.

This research has made extensive use of the NASA/IPAC Extragalactic Database (NED) which is operated by the Jet Propulsion Laboratory, California Institute of Technology, under contract with the National Aeronautics and Space Administration. We also made use of the Lyon-Meudon Extragalactic Database (LEDA; http://leda.univ-lyon1.fr). Based on observations made with the Isaac Newton Group of Telescopes operated on behalf of the UK Particle Physics and Astronomy Research Council (PPARC) and the Nederlandse Organisatie voor Wetenschappelijk Onderzoek (NWO) on the island of Tenerife in the Spanish Observatorio del Roque de Los Muchachos of the Instituto de Astrofísica de Canarias.

Finally, this research was supported by NSF grants AST 9320403 and AST 9803114, and by NASA grant AR0798.01-96A from the Space Telescope Science Institute, operated by the Association of Universities for Research in Astronomy, Inc., under NASA contract NAS5-26555.

\section{REFERENCES}

Afanasiev, V. L., \& Sil'chenko, O. K. 2000, AJ, 119, 126

Ajhar, E. A., Tonry, J. L., Blakeslee, J. P., Riess, A. G., \& Schmidt, B. P. 2001, ApJ, 559, 584

Andersen, V. 1996, AJ, 111, 1805

Ann, H. B. 2001, AJ, 121, 2515

Ashby, M. L. N., Houck, J. R., \& Matthews, K. 1995, ApJ, 447, 545

Athanassoula, E., Morin, S., Wozniak, H., Puy, D., Pierce, M. J., Lombard, J., \& Bosma, A. 1990, MNRAS, 245, 130

Balkowski, C., Charmaraux, P. 1983, A\&AS, 51, 331

Barth, A. J., Ho, L. C., Filippenko, A. V., \& Sargent, W. L. W. 1998, ApJ, 496, 133

Bender, R., \& Möllenhoff, C. 1987, A\&A, 177, 71

Bender, R., Döbereiner, S., \& Möllenhoff, C. 1988, A\&A, 74, 385

Bender, R., Surma, P., Döbereiner, S., Möllenhoff, C., \& Madejski, R. 1989, A\&A, 217, 35

Benedict, G. F., Higdon, J. L., Jeffreys, W. H., Duncombe, R. Hemenway, P. D., Shalus, P. J., Whipple, A. L., Nelan, E., Story, D., McArthur, B., McCartney, J., Franz, O. G., Frederick, L. W., van Altena, Wm. F. 1993, AJ, 105, 1369

Benedict, G. F., Smith, B. J., \& Kenney, J. D. P. 1996, AJ, 111, 1861

Benedict, G. F., Howell, D. A., Jørgensen, I., Kenney, J. D. P., \& Smith, B. J. 2002, AJ, 123, 1411

Bertola, F., Cinzano, P., Corsini, E. M., Rix, H.-W., \& Zeilinger, W. W. 1995, ApJ, 448, L13

Bettoni, D., \& Galletta, G. 1997, A\&AS, 124, 61

Burstein, D., Krumm, N., \& Salpeter, E. E. 1987, AJ, 94, 883

Busarello,G., Capaccioli,M., D'Onofrio,M., Longo,G., Richter,G., \& Zaggia,S. 1996, A\&A, 314, 32

Buta, R. 1986, ApJS, 61, 609

Buta, R., \& Crocker, D. A. 1993, AJ, 105,1344

Buta, R. 1995, ApJS, 96, 39

Carollo, C. M., Stiavelli, M., de Zeeuw, P. T., \& Mack, J. 1997, AJ114, 2366

Carollo, C. M., Stiavelli, M., \& Mack, J. 1998, AJ, 116, 68

Carter, D. 1978, MNRAS, 182, 797

Chromey, F. R. 1974, A\&A, 37, 7

Combes, F., Gerin, M., Nakai, N., Kawabe, R., \& Shaw, M. A. 1992, A\&A, 259, L27

Contini, T., Wozniak, H., Considere, S., \& Davoust, E. 1997, A\&A, 318, L51

Contini, T., Considere, S., \& Davoust, E. 1998, A\&AS, 130, 285

Cox, A. L., \& Sparke, L. S. 1996, The Minnesota Lectures on Extragalactic Neutral Hydrogen (San Francisco: ASP), 168 de Vaucouleurs, G. 1957, Handbuch der Physik, 53, 275

de Vaucouleurs, G., de Vaucouleurs, A., Corwin, H. G., Buta, R. J., Paturel, G., \& Fouqué, P. 1991, Third Reference Catalogue of Bright Galaxies (New York: Springer-Verlag) (RC3)

Devereux, N. A. 1989, ApJ, 346, 126

DuPrie, K., \& Schneider, S. E. 1996, AJ, 112, 937

Elmegreen, B., \& Elmegreen, D. 1985, ApJ, 288, 438

Elmegreen, D., Elmegreen, B., Chromey, F. R., Hasselbacher, D. A., \& Bissell, B. A. 1996, AJ, 111, 1880

Erwin, P., \& Sparke, L. S. 1999, ApJ, 512, L37

Erwin, P., \& Sparke, L. S. 2002, AJ, 124, 65 (Paper I)

Eskridge, P. B., \& Pogge, R. W. 1997, ApJ, 486, 259

Eskridge, P. B., Frogel, J. A., Pogge, R. W., Quillen, A. C., Davies, R. L., DePoy, D. L., Houdashelt, M. L., Kuchinski, L. E., Ramírez, S. V., Sellgren, K., Terndrup, D. M., \& Tiede, G. P. 2000, AJ, 119, 536

Ferruit, P., Wilson, A. S., \& Mulchaey, J. 2000, ApJS, 128, 139

Fisher, D. 1994, in Mass-Transfer Induced Activity in Galaxies, ed. I. Shlosman (Cambridge: Cambridge U. Press), 349

Fisher, D., Franx, M., \& Illingworth, G. 1996, ApJ, 459, 110

Fisher, D. 1997, AJ, 113, 950
Forbes, D. A. 1996, AJ, 112, 1409

Friedli, D., \& Martinet, L. 1993, A\&A, 277, 27

Friedli, D., Wozniak, H., Rieke, M., Martinet, L., \& Bratschi, P. 1996, A\&AS, 118, 461

Friedli, D. 1996, in IAU Colloquium 157: Barred Galaxies, ed. R. Buta, B. G. Elmegreen, \& D. A. Crocker (San Francisco: ASP), 378

García-Barreto, J. A., Downes, D., Combes, F., Gerin, M., Magri, C., Carrasco, L. \& Cruz-González, I. 1991, A\&A, 244, 257

García-Barreto, J. A., Downes, D., \& Huchtmeier, W. K. 1994, A\&A, 288, 705

García-Barreto, J. A., Franco, J., Guichard, J., \& Carrillo, R. 1995, ApJ, 451, 156

Gerin, M. \& Casoli, F. 1994, A\&A, 290, 49

Giovanelli, R., Haynes, M. P., Rubin, V. C., \& Ford, W. K. 1986, ApJ, 301, L7

González Delgado, R. M., Pérez, E., Tadhunter, C., Vilchez, J. M., \& Rodrígeuz-Espinosa, J. M. 1997, ApJS, 108, 155

Hickson, P., Kindl, E., \& Auman, J. R. 1989, ApJS, 70, 687

Ho, L. C., Filippenko, A. V., \& Sargent, W. L. W. 1997b, ApJS, 112,315

Ho, L. C., Filippenko, A. V., \& Sargent, W. L. W. 1997b, ApJ, 487, 568

Ho, L. C., Filippenko, A. V., \& Sargent, W. L. W. 1997c, ApJ, 487, 591

Ho, L. C., Filippenko, A. V., Sargent, W. L. W., \& Peng, C. Y. 1997a, ApJS, 112, 391

Hubble, E. 1926, ApJ, 64, 321

Huchtmeier, W. K., \& Richter, O.-G. 1982, A\&A, 109, 331

Huchtmeier, W. K., \& Seiradakis, J. H. 1985, A\&A, 143, 216

Huchtmeier, W. K., \& Richter, O.-G. 1986, A\&AS, 64, 111

Iyomoto, N., Makishima, K., Matsushita, K., Fukazawa, Y., Tashiro, M., \& Ohashi, T. 1998, ApJ, 503, 168

Jedrzejewski, R. I. 1987, MNRAS, 226, 747

Jungwiert B., Combes F., \& Axon, D. J. 1997, A\&AS, 125, 479

Kamphuis, J. J., Sijbring, D., \& van Albada, T. S. 1996, A\&AS, 116,15

Keel, W. C., \& Hummel, E. 1988, A\&A, 194, 90

Kennicut, R. C., \& Edgar, B. K. 1986, ApJ, 300, 132

Kent, S. M. 1983, ApJ, 266, 562

Kent, S. M., 1987, AJ, 93, 1062

Kent, S. M., \& Glaudell, G., 1989, AJ, 98, 1588

Knapen, J. H., Shlosman, I., \& Peletier, R. F. 1999, ApJ, 125, 363

Knapp, G. R., \& Rupen, M. P. 1996, ApJ, 460, 271

Koopmann, R. A. \& Kenney, J. D. P. 1998, ApJ, 497, L75

Kormendy, J., 1977a, ApJ, 214, 359

Kormendy, J., 1977b, ApJ, 217, 406

Kormendy, J., 1979, ApJ, 227, 714

Kormendy, J., 1982, ApJ, 257, 75

Kormendy, J., 1983, ApJ, 275, 529

Kormendy, J., 1993, in Galactic Bulges, IAU Symposium 153, ed. H. Dejonghe \& H. J. Habing (Dordrecht: Kluwer), 209

Kornreich, D. A., Haynes, M. P., Jore, K. P., \& Lovelace, R. V. E. 2001, AJ, 121, 1358

Kornreich, D. A., Haynes, M. P., Lovelace, R. V. E., \& van Zee, L. 2000, AJ, 120, 139

Laine, S., Shlosman, I., Knapen, J. H., \& Peletier, R. F. 2002, ApJ, 567,97

Lambas, D. G., Maddox, S. J., \& Loveday, J. 1992, MNRAS, 258, 404

Larkin, J. E., Armus, L, Knop, R. A., Soifer, B. T., Matthews, K. 1998, ApJS, 114, 59

Lauer, T. R. 1985, ApJS, 57, 473

Leon, S., Combes, F., \& Menon, T. K. 1998, A\&A, 330, 37 
Lu, N. Y., Hoffman, G. L., Groff, T., Roos, T. \& Lamphier, C. 1993, ApJS, 88, 383

Maciejewski, W. 1998, Ph.D. thesis

Maciejewski, W., Teuben, P. J., Sparke, L. S., \& Stone, J. M. 2002, MNRAS, 329, 502

Magrelli, G., Bettoni, D., \& Galletta, G. 1992, MNRAS, 256, 500

Mahon, E. 1992, Ph.D. thesis, U. Florida

Malin, D. F., Zealey, W. J. 1979, S\&T, 57, 354

Malin, D. F., Quinn, P. J., \& Graham, J. A. 1983, ApJ, 272, L5

Malkan, M. A., Gorjian, V., \& Tam, R. 1998, ApJS, 117, 25

Martini, P., Pogge, R. W., Ravindranath, S., \& An, J. H. 2001, ApJ, 562, 139

Merrifield, M. R., \& Kuijken, K. 1995, MNRAS, 274, 933

Michard, R., \& Marchal, J., 1993, A\&AS, 98, 29

Moran,E.C., Barth,A.J.; Kay,L.E., \& Filippenko,A.V. 2000, ApJ, 540, L73

Mulchaey, J. S., Wilson, A. S., \& Tsvetanov, Z. 1996, ApJS, 102, 309

Mulchaey, J. S., Regan, M. W., \& Kundu, A. 1997, ApJS, 110, 299

Munn, J. A., 1992, ApJ, 399, 444

Nagar, N. M., Wilson, A. S., Mulchaey, J. S., \& Gallimore, J. F. 1999, ApJS, 120, 209

Neistein, E., Maoz, D., Rix, H.-W., Tonry, J. L. 1999, AJ, 117, 2666

Nieto, J.-L., \& Bender, R., A\&A, 215, 266

Nieto, J.-L., Bender, R., Poulain, P., \& Surma, P. 1992, A\&A, 257, 97

Niklas, S., Klein, U., Braine, J., \& Wielebinski, R. 1995, A\&AS, 114,21

Nilson, P. 1973, Uppsala General Catalog of Galaxies, Uppsala Astron. Obs. Annals, 5, 1

Nordgren, T. E., Helou, G., Chengalur, J. N., Terzian, Y., \& Khachikian, E. 1995, ApJS, 99, 461

Ohta, K., Hamabe, M., \& Wakamatsu, K.-I. 1990, ApJ, 357, 71

Patsis, P. A., Athanassoula, E., \& Quillen, A. C. 1997, ApJ, 483, 731

Peletier, R. F., \& Christodoulou, D. M. 1993, AJ, 105, 1378

Petitpas, G. R., \& Wilson, C. D. 2002, ApJ, in press (astro-ph/0204413)

Pogge, R. W., \& Eskridge, P. B. 1987, AJ, 93, 291

Pogge, R. W., \& Eskridge, P. B. 1993, AJ, 106, 1405

Quillen, A. C., Frogel, J. A., \& González, R. A., 1994, ApJ, 437, 162

Rauscher, B. J., 1995, AJ, 109, 1608

Ravindranath,S., Ho, L. C., Peng, C. Y., Filippenko, A. V., \& Sargent, W. L. W. 2001, AJ, 122, 653

Regan, M. W., \& Elmegreen, D. M. 1997, AJ, 114,965

Regan, M. W., \& Mulchaey, J. S. 1999, AJ, 117, 2676

Richter, O.-G., \& Huchtmeier, W. K. 1987, A\&AS, 68, 427

Rix, H.-W., \& White, S. D. M. 1990, apj, 362, 52

Rudnick, G. H., \& Rix, H.-W. 1998, AJ, 116, 1163.

Sadakane, K., Yokoo, T., Arimoto, J.-I., Matsumoto, K., Honda, S., Tanabe, K., Wakamatsu, K.-I., Nishida, M., Yoshida, M., Takada-Hidai, M. 1996, PASJ, 48, 51

Sarzi, M., Rix, H.-W., Shields, J. C., Rudnick, G., Ho, L. C., McIntosh, D. H., Filippenko, A. V., \& Sargent, W. L. W. 2001, ApJ, 550, 65

Schechter, P. L., \& Gunn, J. E. 1978, AJ, 83, 1360
Schwarz, U. J. 1985, A\&A, 142, 273

Schweizer, F., \& Seitzer, P. 1988, ApJ, 328, 88

Scorza, C., \& van den Bosch, F. C. 1998, MNRAS, 300, 469

Seifert, W., \& Scorza, C. 1996, A\&A, 310, 75

Shane, W. W. 1980, A\&A, 82, 314

Shane, W. W., \& Krumm, N. 1983, in Internal Kinematics and Dynamics of Galaxies, IAU Symposium No. 100, ed. E. Athanassoula (Dordrecht: Reidel), 105

Shaw, M., Combes, F., Axon, D. J., \& Wright, G. S. 1993, A\&A, 273,31

Shaw, M., Axon, D., Probst, R., \& Gatley, I. 1995, MNRAS, 274, 369

Sheth, K., Regan,, M. W., Vogel, S. N., \& Teuben, P. J. 2000, ApJ, 532,221

Shlosman, I., Frank, J., \& Begelman, M. C. 1989, Nature, 338, 45

Shlosman, I., \& Heller, C. H. 2002, ApJ, 565, 921

Shostak, G. S. 1987, A\&A, 175, 4

Sil'chenko, O. K., \& Burenkov, A. N. 1990, A\&A, 233, 314

Sil'chenko, O. K. 1998, A\&A, 330, 412

Simien, F., \& Prugniel, Ph. 2000, A\&A, 145, 263

Theureau, G., Bottinelli, L, Coudreau-Durand, N., Gouguenheim, L., Hallet, N., Loulergue, M., Paturel, G, \& Teerikopf, P. 1998, A\&AS, 130, 333

Tifft, W. G., \& Cocke, W. J. 1988, ApJS, 67, 1

Tonry, J. L., Dressler, A., Blakeslee, J. P., Ajhar, E. A., Fletcher, A. B., Luppino, G. A., Metzger, M. R., \& Moore, C. B. 2001, ApJ, 546, 681

Tully, R. B., Verheijen, M. A. W., Pierce, M. J., Huang, J.-S., \& Wainscoat, R. J. 1996, AJ, 112, 2471

Ulvestad, J. S. \& Wilson, A. S. 1984, ApJ, 285, 439

Usui, T., Saitō, M., \& Tomita, A. 1998, AJ, 116, 2166

van den Bergh, S. ApJ, 206, 883

van Driel, W., van Woerden, H., Gallagher III, J. S., \& Schwarz, U. J. 1988, A\&A191, 201

van Driel, W., \& van Woerden, H. 1989, A\&A, 225, 317

van Driel, W., \& van Woerden, H. 1991, A\&A, 243, 71

van Morsel, G., Erwin, P., Sparke, L. S., \& Schwarz, U. J. 2002, in preparation

Verheijen, M. A. W., \& Sancisi, R. 2001, A\&A, 370, 765

Véron-Cetty, M.-P., \& Véron, P. 1986, A\&AS, 65, 241

Wardle, M., Knapp, G., 1986, AJ, 91, 23

Watson, D. M., Guptill, M. T., \& Buchholz, L. M. 1994, ApJ, 420, L21

Whitemore, B. C., Lucas, R. A., McElroy, D. B., Steiman-Cameron, T. Y., Sackett, P. D., Olling, R. P. 1990, AJ, 100, 1489

Wiklind, T., Henkel, C., \& Sage, L. J. 1993, A\&A, 271, 71

Wozniak, H., \& Pierce, M. J., 1991, A\&AS, 88, 325

Wozniak, H., Friedli, D., Martinet, L., Martin, P., \& Bratschi, P. 1995, A\&A, 111, 115 (W95)

Yankulova, I. M. 1999, A\&A, 344, 36

Young, J. S., \& Devereux, N. A. 1991, ApJ, 373, 414

Young, J. S., Xie, S., Tacconi, L, Knezek, P., Viscuo, P. Tacconi-Garman, L., Scoville, N., Schneider, S., Schloerb, F. P., Lord, S., Lesser, A., Kenney, J., Huang, Y.-L., Devereux, N., Claussen, M., Case, J., Carpenter, J., Berry, M., \& Allen, L. 1995, ApJS, 98, 219 
TABLE 1

The WIYN Sample

\begin{tabular}{|c|c|c|c|c|c|}
\hline Galaxy & Type (RC3) & $R_{25}\left(^{\prime \prime}\right)$ & $z\left(\mathrm{~km} \mathrm{~s}^{-1}\right)$ & $D(\mathrm{Mpc})$ & Nucleus \\
\hline NGC 718 & $\mathrm{SAB}(\mathrm{s}) \mathrm{a}$ & 71 & 1733 & 22.7 & L2 \\
\hline NGC 936 & $\mathrm{SB}(\mathrm{rs}) 0^{+}$ & 140 & 1430 & $23.0^{\mathrm{a}}$ & abs. \\
\hline NGC 1022 & $\left(\mathrm{R}^{\prime}\right) \mathrm{SB}(\mathrm{s}) \mathrm{a}$ & 72 & 1453 & 18.5 & $\mathrm{SB}^{\mathrm{c}}$ \\
\hline NGC 2273 & $\left(\mathrm{R}^{\prime}\right) \mathrm{SB}(\mathrm{s}) \mathrm{a}$ & 97 & 1840 & 27.3 & $\mathrm{~S} 2$ \\
\hline NGC 2655 & $\mathrm{SAB}(\mathrm{s}) 0 / \mathrm{a}$ & 147 & 1404 & 22.1 & S2 \\
\hline NGC 2681 & $\left(\mathrm{R}^{\prime}\right) \mathrm{SAB}(\mathrm{rs}) 0 / \mathrm{a}$ & 109 & 692 & $17.2^{\mathrm{a}}$ & L1.9 \\
\hline NGC 2685 & (R) $\mathrm{SBO}^{+}$pec & 134 & 883 & 14.4 & $\mathrm{~S} 2 / \mathrm{T} 2:$ \\
\hline NGC 2787 & $\mathrm{SB}(\mathrm{r}) 0^{+}$ & 95 & 696 & $7.5^{\mathrm{a}}$ & L1.9 \\
\hline NGC 2859 & (R)SB(r) $0^{+}$ & 128 & 1687 & 24.2 & $\mathrm{~T} 2:$ \\
\hline NGC 2880 & $\mathrm{SBO}^{-}$ & 62 & 1551 & $21.9^{\mathrm{a}}$ & abs. $^{\mathrm{d}}$ \\
\hline NGC 2950 & $\mathrm{SB}(\mathrm{r}) 0^{0}$ & 80 & 1337 & $14.9^{\mathrm{a}}$ & abs. \\
\hline NGC 2962 & $(\mathrm{R}) \mathrm{SAB}(\mathrm{rs}) 0^{+}$ & 79 & 1966 & $30.0^{\mathrm{e}}$ & $\ldots$ \\
\hline NGC 3032 & $\mathrm{SAB}(\mathrm{rs}) 0^{\circ}$ & 60 & 1533 & $22.0^{\mathrm{a}}$ & $\mathrm{H} \mathrm{II}^{\mathrm{f}}$ \\
\hline NGC 3185 & $(\mathrm{R}) \mathrm{SB}(\mathrm{r}) \mathrm{a}$ & 71 & 1218 & 17.1 & S2: \\
\hline NGC 3412 & $\mathrm{SB}(\mathrm{s}) 0^{0}$ & 109 & 865 & $11.3^{\mathrm{a}}$ & abs. \\
\hline NGC 3489 & $\mathrm{SAB}(\mathrm{rs}) 0^{+}$ & 106 & 708 & $12.1^{\mathrm{a}}$ & $\mathrm{T} 2 / \mathrm{S} 2$ \\
\hline IC 676 & (R) SB(r) $0^{+}$ & 74 & 1290 & 19.0 & $\mathrm{SB}^{\mathrm{g}}$ \\
\hline NGC 3729 & $\mathrm{SB}(\mathrm{r}) \mathrm{a}$ pec & 85 & 1024 & 16.4 & H II \\
\hline NGC 3941 & $\mathrm{SB}(\mathrm{s}) 0^{0}$ & 104 & 928 & $12.2^{\mathrm{a}}$ & S2: \\
\hline NGC 3945 & (R)SB $(\mathrm{rs}) 0^{+}$ & 157 & 1220 & 19.3 & $\mathrm{~L} 2$ \\
\hline NGC 4045 & $\mathrm{SAB}(\mathrm{r}) \mathrm{a}$ & 81 & 1981 & 26.3 & $\ldots$ \\
\hline NGC 4143 & $\mathrm{SAB}(\mathrm{s}) 0^{\circ}$ & 69 & 985 & $15.9^{\mathrm{a}}$ & L1.9 \\
\hline NGC 4203 & $\mathrm{SABO}^{-}:$ & 102 & 1086 & $15.1^{\mathrm{a}}$ & L1.9 \\
\hline NGC 4245 & $\mathrm{SB}(\mathrm{r}) 0 / \mathrm{a}:$ & 87 & 890 & $12.0^{\mathrm{b}}$ & H II \\
\hline NGC 4310 & $\left(\mathrm{R}^{\prime}\right) \mathrm{SABO}^{+} ?$ & 64 & 913 & $12.0^{\mathrm{b}}$ & $\ldots$ \\
\hline NGC 4314 & $\mathrm{SB}(\mathrm{rs}) \mathrm{a}:$ & 125 & 963 & $12.0^{\mathrm{b}}$ & L2 \\
\hline NGC 4386 & $\mathrm{SAB}^{0}:$ & 74 & 1677 & $27.0^{\mathrm{a}}$ & $\ldots$ \\
\hline NGC 4643 & $\mathrm{SB}(\mathrm{rs}) 0 / \mathrm{a}$ & 93 & 1319 & 17.8 & $\mathrm{~T} 2$ \\
\hline NGC 4665 & $\mathrm{SB}(\mathrm{s}) 0 / \mathrm{a}$ & 114 & 785 & 10.4 & abs. \\
\hline NGC 4691 & (R) SB(s)0/a pec & 85 & 1110 & 14.6 & $\mathrm{H} \mathrm{II}^{\mathrm{h}}$ \\
\hline NGC 5338 & SB0: & 76 & 816 & $12.8^{\mathrm{a}}$ & $\ldots$ \\
\hline NGC 5377 & $(\mathrm{R}) \mathrm{SB}(\mathrm{s}) \mathrm{a}$ & 112 & 1793 & 26.7 & L2 \\
\hline NGC 5701 & (R) $\mathrm{SB}(\mathrm{rs}) 0 / \mathrm{a}$ & 128 & 1505 & 20.9 & T2: \\
\hline NGC 5750 & $\mathrm{SB}(\mathrm{r}) 0 / \mathrm{a}$ & 91 & 1687 & 26.2 & $\mathrm{~L} / \mathrm{S} 2^{\mathrm{i}}$ \\
\hline NGC 6654 & $\left(\mathrm{R}^{\prime}\right) \mathrm{SB}(\mathrm{s}) 0 / \mathrm{a}$ & 79 & 1821 & 28.2 & abs. \\
\hline UGC 11920 & $\mathrm{SB} 0 / \mathrm{a}$ & 72 & 1145 & 18.5 & $\ldots$ \\
\hline NGC 7280 & (R) SAB $(\mathrm{r}) 0^{+}$ & 66 & 1844 & $24.3^{\mathrm{a}}$ & $\ldots$ \\
\hline NGC 7743 & (R) SB(s) $0^{+}$ & 91 & 1710 & $20.7^{\mathrm{a}}$ & S2 \\
\hline
\end{tabular}

Note. $-R_{25}$ is one-half of $D_{25}$, from RC3; heliocentric redshift $z$ is from NED; distance $D$ is from LEDA (redshift, corrected for Virgo-centric infall, $+H_{0}=75$ $\mathrm{km} \mathrm{s}^{-1} \mathrm{Mpc}^{-1}$ ), except as noted. Nuclear classifications are from Ho, Filippenko, \& Sargent (1997b), unless otherwise indicated: L = LINER, $\mathrm{S}=$ Seyfert, $\mathrm{T}=$ transition between LINER and H II, H II = H II region, SB = starburst, "abs." = absorption lines only. Uncertain classifications are indicated by a trailing colon.

a Tonry et al. (2001)

${ }^{\mathrm{b}}$ Forbes (1996)

${ }^{\mathrm{c}}$ Ashby, Houck, \& Matthews (1995).

d spectra of Munn (1992) show no emission.

e Ajhar et al. (2001)

${ }^{\mathrm{f}}$ Véron-Cetty \& Véron (1986)

${ }^{g}$ Contini, Considere, \& Davoust (1998)

${ }^{\mathrm{h}}$ Chromey (1974), García-Barreto et al. (1995)

${ }^{\mathrm{i}}$ Véron-Cetty \& Véron (1986) 
TABLE 2

OBservations

\begin{tabular}{|c|c|c|c|c|}
\hline Galaxy & $\begin{array}{l}\text { Telescope/ } \\
\text { Instrument }\end{array}$ & Filters & Seeing $\left(\mathrm{R},{ }^{\prime \prime}\right)$ & Notes \\
\hline NGC 718 & WIYN & $\mathrm{BR}$ & 1.0 & \\
\hline NGC 936 & $\begin{array}{l}\text { DSS } \\
\text { WHT } \\
\text { WFPC2 }\end{array}$ & $\begin{array}{l}\text { IIIaJ } \\
\text { BVR } \\
555 \mathrm{~W}\end{array}$ & 0.85 & \\
\hline NGC 1022 & WIYN & $\mathrm{BR}$ & 1.3 & \\
\hline NGC 2273 & $\begin{array}{l}\text { WIYN } \\
\text { WFPC2 } \\
\text { NIC1/2/3 }\end{array}$ & $\begin{array}{l}\text { UBRI } \\
606 \mathrm{~W}, 791 \mathrm{~W} \\
160 \mathrm{~W}, 164 \mathrm{~N}, 222 \mathrm{M}\end{array}$ & 0.65 & \\
\hline NGC 2655 & $\begin{array}{l}\text { WIYN } \\
\text { WFPC2 }\end{array}$ & $\begin{array}{l}\text { UBR } \\
547 \mathrm{M}\end{array}$ & 0.8 & non-lin. CCD \\
\hline NGC 2681 & $\begin{array}{l}\text { WIYN } \\
\text { PC1 } \\
\text { NIC3 }\end{array}$ & $\begin{array}{l}\text { BR } \\
555 \mathrm{~W} \\
160 \mathrm{~W}, 187 \mathrm{~N}\end{array}$ & 0.8 & \\
\hline NGC 2685 & $\begin{array}{l}\text { WIYN } \\
\text { NIC3 }\end{array}$ & $\begin{array}{l}\mathrm{BR} \\
160 \mathrm{~W}, 187 \mathrm{~N}\end{array}$ & 0.65 & \\
\hline NGC 2787 & $\begin{array}{l}\text { WIYN } \\
\text { WFPC2 } \\
\text { NIC3 }\end{array}$ & $\begin{array}{l}\mathrm{BR} \\
555 \mathrm{~W}, 702 \mathrm{~W}, 814 \mathrm{~W} \\
160 \mathrm{~W}, 187 \mathrm{~N}\end{array}$ & 0.9 & non-lin. CCD \\
\hline NGC 2859 & WIYN & UBR & 0.6 & non-lin. CCD \\
\hline NGC 2880 & $\begin{array}{l}\text { WIYN } \\
\text { WFPC2 }\end{array}$ & $\begin{array}{l}\mathrm{BR} \\
450 \mathrm{~W}, 555 \mathrm{~W}, 814 \mathrm{~W}\end{array}$ & 0.7 & \\
\hline NGC 2950 & $\begin{array}{l}\text { WIYN } \\
\text { WFPC2 }\end{array}$ & $\begin{array}{l}\text { UBR } \\
450 \mathrm{~W}, 555 \mathrm{~W}, 702 \mathrm{~W}, 814 \mathrm{~W}\end{array}$ & 0.65 & non-lin. CCD \\
\hline NGC 2962 & $\begin{array}{l}\text { WIYN } \\
\text { WFCP2 }\end{array}$ & $\begin{array}{l}\mathrm{BR} \\
814 \mathrm{~W}\end{array}$ & 1.2 & \\
\hline NGC 3032 & $\begin{array}{l}\text { WIYN } \\
\text { WFPC2 } \\
\text { NIC2 }\end{array}$ & $\begin{array}{l}\text { UBR } \\
606 \mathrm{~W} \\
160 \mathrm{~W}\end{array}$ & 0.8 & non-lin. CCD \\
\hline NGC 3185 & WIYN & $\mathrm{BR}$ & 0.9 & non-lin. CCD \\
\hline NGC 3412 & $\begin{array}{l}\text { WIYN } \\
\text { WFPC2 }\end{array}$ & $\begin{array}{l}\text { UBR } \\
606 W\end{array}$ & 1.15 & non-lin. CCD \\
\hline NGC 3489 & $\begin{array}{l}\text { WIYN } \\
\text { WFPC2 }\end{array}$ & $\begin{array}{l}\text { UBR } \\
555 \mathrm{~W}, 606 \mathrm{~W}, 814 \mathrm{~W}\end{array}$ & 0.85 & \\
\hline IC 676 & WIYN & $\mathrm{BR}$ & 0.95 & \\
\hline NGC 3729 & $\begin{array}{l}\text { WIYN } \\
\text { MK }\end{array}$ & $\begin{array}{l}\mathrm{R} \\
\mathrm{BR}\end{array}$ & $\begin{array}{l}1.0 \\
1.3\end{array}$ & \\
\hline NGC 3941 & WIYN & $\mathrm{BR}$ & 0.8 & \\
\hline NGC 3945 & $\begin{array}{l}\text { WIYN } \\
\text { WFPC2 }\end{array}$ & $\begin{array}{l}\mathrm{BR} \\
450 \mathrm{~W}, 555 \mathrm{~W}, 814 \mathrm{~W}\end{array}$ & 0.65 & \\
\hline NGC 4045 & WIYN & $\mathrm{BR}$ & 0.85 & \\
\hline NGC 4143 & $\begin{array}{l}\text { WIYN } \\
\text { WFPC2 } \\
\text { NIC2 }\end{array}$ & $\begin{array}{l}\text { BR } \\
218 \mathrm{~W}, 606 \mathrm{~W} \\
160 \mathrm{~W}\end{array}$ & 0.75 & \\
\hline NGC 4203 & $\begin{array}{l}\text { WIYN } \\
\text { WFPC2 }\end{array}$ & $\begin{array}{l}\mathrm{BR} \\
218 \mathrm{~W}, 555 \mathrm{~W}, 814 \mathrm{~W}\end{array}$ & 0.95 & \\
\hline
\end{tabular}


TABle 2-Continued

\begin{tabular}{|c|c|c|c|c|}
\hline Galaxy & $\begin{array}{l}\text { Telescope/ } \\
\text { Instrument }\end{array}$ & Filters & Seeing $\left(\mathrm{R},{ }^{\prime \prime}\right)$ & Notes \\
\hline NGC 4245 & $\begin{array}{l}\text { WIYN } \\
\text { WFPC2 }\end{array}$ & $\begin{array}{l}\mathrm{BR} \\
606 \mathrm{~W}\end{array}$ & 0.7 & \\
\hline NGC 4310 & WIYN & $\mathrm{BR}$ & 0.7 & \\
\hline NGC 4314 & $\begin{array}{l}\text { DSS } \\
\text { WFPC2 } \\
\text { NIC2 }\end{array}$ & $\begin{array}{l}103 \mathrm{aE} \\
336 \mathrm{~W}, 439 \mathrm{~W}, 569 \mathrm{~W}, 814 \mathrm{~W} \\
160 \mathrm{~W}\end{array}$ & & \\
\hline NGC 4386 & WIYN & $\mathrm{BR}$ & 0.8 & \\
\hline NGC 4643 & WIYN & $\mathrm{BR}$ & 1.3 & non-lin. CCD \\
\hline NGC 4665 & WIYN & $\mathrm{BR}$ & 1.2 & \\
\hline NGC 4691 & WIYN & $\mathrm{BR}$ & 1.2 & non-lin. CCD \\
\hline NGC 5338 & WIYN & $\mathrm{BR}$ & 0.7 & \\
\hline NGC 5377 & $\begin{array}{l}\text { WIYN } \\
\text { WFPC2 } \\
\text { NIC2 }\end{array}$ & $\begin{array}{l}\mathrm{BR} \\
606 \mathrm{~W} \\
110 \mathrm{~W}, 160 \mathrm{~W}\end{array}$ & 1.1 & non-lin. CCD \\
\hline NGC 5701 & WIYN & VBR & 0.5 & non-lin. CCD \\
\hline NGC 5750 & WIYN & $\mathrm{BR}$ & 1.3 & non-lin. CCD \\
\hline NGC 6654 & $\begin{array}{l}\text { WIYN } \\
\text { WFPC2 }\end{array}$ & $\begin{array}{l}\mathrm{BR} \\
450 \mathrm{~W}, 814 \mathrm{~W}\end{array}$ & 0.9 & non-lin. CCD \\
\hline UGC 11920 & WIYN & $\mathrm{BR}$ & 0.85 & non-lin. CCD \\
\hline NGC 7280 & $\begin{array}{l}\text { WIYN } \\
\text { WFPC2 } \\
\text { NIC2 }\end{array}$ & $\begin{array}{l}\mathrm{BR} \\
606 \mathrm{~W} \\
160 \mathrm{~W}\end{array}$ & 0.6 & \\
\hline NGC 7743 & $\begin{array}{l}\text { WIYN } \\
\text { WFPC2 } \\
\text { NIC2 }\end{array}$ & $\begin{array}{l}\mathrm{BR} \\
547 \mathrm{M}, 606 \mathrm{~W} \\
160 \mathrm{~W}\end{array}$ & 0.65 & \\
\hline
\end{tabular}

Note. - Image sources: WIYN $=$ Wisconsin-Indiana-Yale-NOAO Telescope, WHT $=$ William Herschel Telescope, MK = Mauna Kea 2.24m Telescope (Tully et al. 1996), DSS $=$ Digitized Sky Survey, PC1 = Planetary Camera of HST (pre-refurbishment), WFPC2 = Wide Field/Planetary Camera-2 of HST, NIC1/2/3 = NICMOS1, 2, or 3 camera of HST. Seeing for WIYN, WHT, or MK observations is FWHM, in arc seconds, of stars in the best $R$-band image (Moffat profile). "non-lin. CCD" indicates WIYN images were taken with the CCD before the non-linearity was fixed (see text for details). 
TABle 3

Comparison of Bar Length Measurements

\begin{tabular}{clllcl}
\hline \hline Galaxy & $a_{\max }$ & $a_{10}$ & $a_{\min }$ & Other Measurements & Source for Other Measurements \\
\hline NGC 936 & $41^{\prime \prime}$ & 51 & 60 & 41 & Kormendy (1983) \\
& & & & 52 & Kent (1987) \\
& & & & 50 & Kent \& Glaudell (1989) \\
NGC 4314 & 70 & \multirow{2}{*}{113} & \multirow{2}{*}{127} & 71 & Elmegreen \& Elmegreen (1985) \\
& & & & 60 & Quillen et al. (1994) \\
& & & & 66 & Shaw et al. (1995) \\
NGC 4643 & 50 & 62 & 69 & 51 & Ohta et al. (1990) \\
& & & & 58 & Shaw et al. (1995) \\
NGC 4665 & 45 & 65 & 99 & & \\
\hline
\end{tabular}

TABLE 4

Model Bar Length Measurements

\begin{tabular}{llcccl}
\hline \hline Component & & $a_{\max }$ & $a_{10}$ & $a_{\min }$ & Ferrers Bar $a$ \\
\hline Model 1: & Inner Bar & 1.82 & 2.11 & 2.11 & 4.2 \\
& Outer Bar & 3.61 & $\cdots$ & $\cdots$ & 7.0 \\
\multirow{2}{*}{ Model 2: } & Inner Bar & 0.62 & 0.92 & 0.87 & 1.2 \\
& Outer Bar & 3.60 & $\cdots$ & $\cdots$ & 6.0 \\
\hline
\end{tabular}


TABle 5

Observed Structures

\begin{tabular}{|c|c|c|c|c|c|c|c|}
\hline Galaxy & $i$ & Rot. & Notes & Structure & $\underset{\prime \prime}{\text { Size }}$ & $\underset{0}{\mathrm{PA}}$ & $e_{\max }$ \\
\hline NGC 718 & 30 & + & $\mathrm{DB}, \mathrm{NR}$ & $\begin{array}{l}\text { Outer Disk } \\
\text { Bar 1 } \\
\text { NR(B) } \\
\text { Bar 2 }\end{array}$ & $\begin{array}{r}71 \\
20-30 \\
\sim 3.3 \\
1.6-3.6\end{array}$ & $\begin{array}{r}5 \\
152(158) \\
\sim 50 \\
\sim 15\end{array}$ & $\begin{array}{r}0.13 \\
0.23 \\
\sim 0.15 \\
0.19\end{array}$ \\
\hline NGC 936 & 41 & $-?$ & NR & $\begin{array}{l}\text { Outer Disk } \\
\text { Bar } \\
\text { NR(S) }\end{array}$ & $\begin{array}{r}140 \\
41-51 \\
\sim 8\end{array}$ & $\begin{array}{r}130 \\
78(81) \\
\sim 130\end{array}$ & $\begin{array}{r}0.24 \\
0.47 \\
\sim 0.23\end{array}$ \\
\hline NGC 1022 & $\lesssim 24$ & + & dusty & $\begin{array}{l}\text { Outer Disk } \\
\text { Inner Ring } \\
\text { Bar }\end{array}$ & $\begin{array}{r}72 \\
\sim 30 \\
19-22\end{array}$ & $\begin{array}{r}\sim 174 \\
\sim 60 \\
\sim 115\end{array}$ & $\begin{array}{l}0.08 \\
0.16 \\
0.51\end{array}$ \\
\hline NGC 2273 & 50 & + & NS, NR & $\begin{array}{l}\text { [Outer Disk] } \\
\text { Outer Ring } \\
\text { Bar } \\
\text { NR(SF) }\end{array}$ & $\begin{array}{r}97 \\
145 \\
14-17 \\
2.2\end{array}$ & $\begin{array}{r}50 \\
50 \\
116(109) \\
\sim 30\end{array}$ & $\begin{array}{r}\sim 0.34 \\
0.45 \\
0.43 \\
0.47\end{array}$ \\
\hline NGC 2655 & 26 & $-?$ & dusty, OPG & $\begin{array}{l}\text { Outer Disk } \\
\text { Bar(?) }\end{array}$ & $\begin{array}{r}147 \\
8.7-63\end{array}$ & $\begin{array}{r}\sim 95 \\
90\end{array}$ & $\begin{array}{l}0.10 \\
0.35\end{array}$ \\
\hline NGC 2681 & $\sim 18$ & - & $\mathrm{DB}, \mathrm{NR}$ & $\begin{array}{l}\text { Outer Disk } \\
\text { Bar 1 } \\
\text { NR(B) } \\
\text { Bar } 2 \\
\text { Bar } 3\end{array}$ & $\begin{array}{r}109 \\
50-60 \\
18 \\
18-19 \\
1.7-3.3\end{array}$ & $\begin{array}{r}140 \\
30 \\
73 \\
20\end{array}$ & $\begin{array}{r}0.05 \\
0.23 \\
0.0 \\
0.33 \\
0.26\end{array}$ \\
\hline NGC 2685 & $\ldots a$ & $\cdots$ & $\mathrm{ID}, \mathrm{OPG}$ & $\begin{array}{l}\text { [Outer Disk] } \\
\text { Outer Ring } \\
\text { Bar? } \\
\text { Disk }\end{array}$ & $\begin{array}{r}134 \\
155 \\
33-54 \\
1.8-3.7\end{array}$ & $\begin{array}{l}38 \\
25 \\
38 \\
35\end{array}$ & $\begin{array}{r}\sim 0.54 \\
0.50 \\
0.61 \\
0.61\end{array}$ \\
\hline NGC 2787 & 55 & + & dusty, ID, OPG & $\begin{array}{l}\text { Outer Disk } \\
\text { Bar } \\
\text { Disk }\end{array}$ & $\begin{array}{r}95 \\
29-36 \\
18-21\end{array}$ & $\begin{array}{r}109 \\
160(150) \\
113\end{array}$ & $\begin{array}{l}0.41 \\
0.34 \\
0.34\end{array}$ \\
\hline NGC 2859 & $\sim 25$ & + & DB, NR & $\begin{array}{l}\text { [Outer Disk] } \\
\text { Outer Ring } \\
\text { Bar 1 } \\
\text { NR }(\mathrm{R}) \\
\text { Bar } 2\end{array}$ & $\begin{array}{r}128 \\
120 \\
34-43 \\
7.0 \\
4.1-6.2\end{array}$ & $\begin{array}{r}\sim 90 \\
83 \\
162 \\
\sim 60 \\
62\end{array}$ & $\begin{array}{r}\sim 0.1 \\
0.23 \\
0.40 \\
\sim 0.2 \\
0.31\end{array}$ \\
\hline NGC 2880 & 52 & $-?$ & ID & $\begin{array}{l}\text { Outer Disk } \\
\text { Bar } \\
\text { Disk }\end{array}$ & $\begin{array}{r}62 \\
8.0-9.0 \\
3.0-4.5\end{array}$ & $\begin{array}{r}144 \\
82(105) \\
138\end{array}$ & $\begin{array}{l}0.37 \\
0.20 \\
0.22\end{array}$ \\
\hline NGC 2950 & 48 & + & $\mathrm{DB}, \mathrm{NR}$ & $\begin{array}{l}\text { Outer Disk } \\
\text { Bar 1 } \\
\text { NR(S) } \\
\text { Bar } 2\end{array}$ & $\begin{array}{r}80 \\
24-31 \\
\sim 4.2 \\
3.2-3.9\end{array}$ & $\begin{array}{r}120 \\
162(155) \\
\sim 120 \\
85(92)\end{array}$ & $\begin{array}{r}0.32 \\
0.43 \\
\sim 0.3 \\
0.36\end{array}$ \\
\hline NGC 2962 & 51 & - & DB & $\begin{array}{l}\text { Outer Disk } \\
\text { Bar } 1 \\
\text { Bar } 2\end{array}$ & $\begin{array}{r}79 \\
29-43 \\
3.5-4.2\end{array}$ & $\begin{array}{r}10 \\
168(174) \\
93(10)\end{array}$ & $\begin{array}{l}0.35 \\
0.30 \\
0.03\end{array}$ \\
\hline NGC 3032 & 30 & - & NS & $\begin{array}{l}\text { Outer Disk } \\
\text { Bar? }\end{array}$ & $\begin{array}{r}60 \\
7.9-11\end{array}$ & $\begin{array}{l}95 \\
97\end{array}$ & $\begin{array}{l}0.13 \\
0.20\end{array}$ \\
\hline NGC 3185 & 48 & + & dusty & $\begin{array}{l}\text { Outer Disk } \\
\text { Inner Ring } \\
\text { Bar } \\
\text { NR }(R) ?\end{array}$ & $\begin{array}{r}71 \\
39 \\
31-32 \\
\sim 4\end{array}$ & $\begin{array}{r}140 \\
125 \\
114 \\
\sim 140\end{array}$ & $\begin{array}{r}0.31 \\
0.62 \\
0.58 \\
\sim 0.3\end{array}$ \\
\hline NGC 3412 & 52 & $\cdots$ & ID & $\begin{array}{l}\text { Outer Disk } \\
\text { Bar } \\
\text { Disk }\end{array}$ & $\begin{array}{r}109 \\
15-21 \\
1.0-6.1\end{array}$ & $\begin{array}{r}153 \\
100(115) \\
154\end{array}$ & $\begin{array}{l}0.37 \\
0.26 \\
0.33\end{array}$ \\
\hline
\end{tabular}


TABLE 5-Continued

\begin{tabular}{|c|c|c|c|c|c|c|c|}
\hline Galaxy & $\begin{array}{l}i \\
\circ\end{array}$ & Rot. & Notes & Structure & $\underset{\prime \prime}{\text { Size }}$ & $\underset{\circ}{\mathrm{PA}}$ & $e_{\max }$ \\
\hline NGC 3489 & 56 & $+?$ & dusty, NR & $\begin{array}{l}\text { Outer Disk } \\
\text { Outer Ring } \\
\text { Bar? } \\
\text { NR(R) }\end{array}$ & $\begin{array}{r}106 \\
51 \\
\sim 10 \\
3.2\end{array}$ & $\begin{array}{r}71 \\
71 \\
\sim 20(\sim 55) \\
\sim 60\end{array}$ & $\begin{array}{r}0.42 \\
0.51 \\
\sim 0.17 \\
\sim 0.7\end{array}$ \\
\hline IC 676 & 44 & $-?$ & dusty & $\begin{array}{l}\text { Outer Disk } \\
\text { Outer Ring } \\
\text { Bar }\end{array}$ & $\begin{array}{r}74 \\
55 \\
13-34\end{array}$ & $\begin{array}{r}15 \\
8 \\
164\end{array}$ & $\begin{array}{l}0.27 \\
0.32 \\
0.72\end{array}$ \\
\hline NGC 3729 & $\sim 50$ & + & dusty & $\begin{array}{l}\text { Outer Disk } \\
\text { Inner Ring } \\
\text { Bar }\end{array}$ & $\begin{array}{r}85 \\
51 \\
23-26\end{array}$ & $\begin{array}{r}\sim 170 \\
167 \\
26\end{array}$ & $\begin{array}{r}\sim 0.35 \\
0.50 \\
0.66\end{array}$ \\
\hline NGC 3941 & 51 & $+?$ & $\mathrm{DB}, \mathrm{OPG}$ & $\begin{array}{l}\text { Outer Disk } \\
\text { Bar } 1 \\
\text { Bar } 2\end{array}$ & $\begin{array}{r}104 \\
21-32 \\
3.2-4.4\end{array}$ & $\begin{array}{r}10 \\
166(176) \\
35(20)\end{array}$ & $\begin{array}{r}\sim 0.35 \\
0.47 \\
0.21\end{array}$ \\
\hline NGC 3945 & $\sim 50$ & $-?$ & $\mathrm{DB}, \mathrm{NR}, \mathrm{ID}$ & $\begin{array}{l}\text { [Outer Disk] } \\
\text { Outer Ring } \\
\text { Bar 1 } \\
\text { Disk } \\
\text { NR(S) } \\
\text { Bar 2 }\end{array}$ & $\begin{array}{r}157 \\
150 \\
32-39 \\
10-18 \\
6.5 \\
2.6-3.0\end{array}$ & $\begin{array}{r}158 \\
160 \\
72(75) \\
158 \\
158 \\
90(115)\end{array}$ & $\begin{array}{r}\sim 0.35 \\
0.47 \\
0.29 \\
0.36 \\
\sim 0.57 \\
0.11\end{array}$ \\
\hline NGC 4045 & 48 & + & dusty & $\begin{array}{l}\text { Outer Disk } \\
\text { Outer Ring } \\
\text { Bar }\end{array}$ & $\begin{array}{r}81 \\
66 \\
18-20\end{array}$ & $\begin{array}{r}90 \\
90 \\
\sim 18\end{array}$ & $\begin{array}{l}0.32 \\
0.39 \\
0.30\end{array}$ \\
\hline NGC 4143 & 59 & + & NS, ID & $\begin{array}{l}\text { Outer Disk } \\
\text { Bar } \\
\text { Disk }\end{array}$ & $\begin{array}{r}69 \\
17-28 \\
4.2-6.2\end{array}$ & $\begin{array}{r}144 \\
163(156) \\
142\end{array}$ & $\begin{array}{r}0.46 \\
\sim 0.38 \\
\\
0.25\end{array}$ \\
\hline NGC 4203 & 34 & + & OPG & $\begin{array}{l}\text { Outer Disk } \\
\text { Bar }\end{array}$ & $\begin{array}{r}102 \\
13-46\end{array}$ & $\begin{array}{r}10 \\
9\end{array}$ & $\begin{array}{l}0.17 \\
0.24\end{array}$ \\
\hline NGC 4245 & 40 & + & NS, NR & $\begin{array}{l}\text { Outer Disk } \\
\text { Bar } \\
\text { NR(SF) }\end{array}$ & $\begin{array}{r}87 \\
37-42 \\
4.2\end{array}$ & $\begin{array}{r}\sim 0 \\
137 \\
\sim 145\end{array}$ & $\begin{array}{r}0.25 \\
0.48 \\
\sim 0.15\end{array}$ \\
\hline NGC 4310 & 62 & $\cdots$ & dusty & $\begin{array}{l}\text { Outer Disk } \\
\text { Bar? }\end{array}$ & $\begin{array}{r}64 \\
13-28\end{array}$ & $\begin{array}{l}159 \\
161\end{array}$ & $\begin{array}{r}0.5 \\
0.63\end{array}$ \\
\hline NGC 4314 & 25 & + & $\mathrm{DB}, \mathrm{NR}, \mathrm{NS}$ & $\begin{array}{l}\text { Outer Disk } \\
\text { Bar } 1 \\
\text { NR(SF) } \\
\text { Bar } 2\end{array}$ & $\begin{array}{r}125 \\
70-113 \\
\sim 6.8 \\
4.5-5.6\end{array}$ & $\begin{array}{r}65 \\
146 \\
\sim 135 \\
136\end{array}$ & $\begin{array}{r}0.09 \\
0.65 \\
\sim 0.24 \\
0.23\end{array}$ \\
\hline NGC 4386 & 48 & $\cdots$ & ID & $\begin{array}{l}\text { Outer Disk } \\
\text { Bar } \\
\text { Disk }\end{array}$ & $\begin{array}{r}74 \\
25-36 \\
2.4-3.2\end{array}$ & $\begin{array}{l}140 \\
134 \\
141\end{array}$ & $\begin{array}{l}0.31 \\
0.52 \\
0.28\end{array}$ \\
\hline NGC 4643 & 38 & $+?$ & NS, ID & $\begin{array}{l}\text { Outer Disk } \\
\text { Bar } \\
\text { Disk }\end{array}$ & $\begin{array}{r}93 \\
50-62 \\
3.5-5.4\end{array}$ & $\begin{array}{r}55 \\
133 \\
53\end{array}$ & $\begin{array}{l}0.20 \\
0.45 \\
0.10\end{array}$ \\
\hline NGC 4665 & 33 & + & & $\begin{array}{l}\text { Outer Disk } \\
\text { Bar }\end{array}$ & $\begin{array}{r}114 \\
45-65\end{array}$ & $\begin{array}{r}120 \\
4\end{array}$ & $\begin{array}{l}0.15 \\
0.51\end{array}$ \\
\hline NGC 4691 & 42 & $\cdots$ & dusty & $\begin{array}{l}\text { Outer Disk } \\
\text { Bar }\end{array}$ & $\begin{array}{r}85 \\
30-55\end{array}$ & $\begin{array}{l}30 \\
82\end{array}$ & $\begin{array}{l}0.24 \\
0.64\end{array}$ \\
\hline NGC 5338 & 68 & $\cdots$ & dusty & $\begin{array}{l}\text { Outer Disk } \\
\text { Bar }\end{array}$ & $\begin{array}{r}76 \\
11-15\end{array}$ & $\begin{array}{r}95 \\
125\end{array}$ & $\begin{array}{l}0.58 \\
0.46\end{array}$ \\
\hline
\end{tabular}


TABLE 5-Continued

\begin{tabular}{|c|c|c|c|c|c|c|c|}
\hline Galaxy & $\begin{array}{l}i \\
\circ\end{array}$ & Rot. & Notes & Structure & $\underset{\prime \prime}{\text { Size }}$ & $\underset{\circ}{\mathrm{PA}}$ & $e_{\max }$ \\
\hline NGC 5377 & $\sim 55$ & - & NS, NR & $\begin{array}{l}\text { [Outer Disk] } \\
\text { Outer Ring } \\
\text { Inner Ring } \\
\text { Bar } \\
\text { NR }(R) \\
\text { NR(B) }\end{array}$ & $\begin{array}{r}111 \\
150 \\
\sim 70 \\
58-78 \\
6.5 \\
\sim 3\end{array}$ & $\begin{aligned} \sim & 35 \\
& 27 \\
& 43 \\
& 45 \\
\sim & 30 \\
\sim & 30\end{aligned}$ & $\begin{array}{r}\sim 0.4 \\
0.47 \\
0.64 \\
0.66 \\
\sim 0.45 \\
0.41\end{array}$ \\
\hline NGC 5701 & $\sim 20$ & - & NS & $\begin{array}{l}\text { [Outer Disk] } \\
\text { Outer Ring } \\
\text { Bar }\end{array}$ & $\begin{array}{r}128 \\
\sim 125 \\
40-58\end{array}$ & $\begin{array}{r}\sim 45 \\
78 \\
177\end{array}$ & $\begin{array}{r}\sim 0.05 \\
0.20 \\
0.43\end{array}$ \\
\hline NGC 5750 & 62 & - & dusty & $\begin{array}{l}\text { Outer Disk } \\
\text { Bar }\end{array}$ & $\begin{array}{r}91 \\
20-24\end{array}$ & $\begin{array}{r}65 \\
121\end{array}$ & $\begin{array}{l}0.50 \\
0.37\end{array}$ \\
\hline NGC 6654 & 44 & - & DB & $\begin{array}{l}\text { Outer Disk } \\
\text { Bar } 1 \\
\text { Bar } 2\end{array}$ & $\begin{array}{r}79 \\
26-38 \\
2.7-4.2\end{array}$ & $\begin{array}{r}0 \\
17 \\
135(160)\end{array}$ & $\begin{array}{l}0.27 \\
0.51 \\
0.15\end{array}$ \\
\hline UGC 11920 & 52 & - & dusty & $\begin{array}{l}\text { Outer Disk } \\
\text { Bar } \\
\text { Bar 2? }\end{array}$ & $\begin{array}{r}72 \\
26-39 \\
2.5-3.3\end{array}$ & $\begin{array}{r}50 \\
45 \\
0(17)\end{array}$ & $\begin{array}{l}0.36 \\
0.51 \\
0.19\end{array}$ \\
\hline NGC 7280 & 49 & $+?$ & $\mathrm{DB}, \mathrm{OPG}$ & $\begin{array}{l}\text { Outer Disk } \\
\text { Bar } 1 \\
\text { Bar } 2\end{array}$ & $\begin{array}{r}66 \\
11-27 \\
1.2-1.6\end{array}$ & $\begin{array}{r}73 \\
55(63) \\
115(101)\end{array}$ & $\begin{array}{l}0.33 \\
0.40 \\
0.30\end{array}$ \\
\hline NGC 7743 & 28 & - & NS & $\begin{array}{l}\text { Outer Disk } \\
\text { Bar } 1\end{array}$ & $\begin{array}{r}91 \\
31-58\end{array}$ & $\begin{array}{r}105 \\
96\end{array}$ & $\begin{array}{l}0.11 \\
0.51\end{array}$ \\
\hline
\end{tabular}

Note. - For each galaxy, we give disk inclination and rotation, along with a summary of notable features: $\mathrm{DB}=$ double barred; $\mathrm{ID}=$ inner disk (possible secondary bar); $\mathrm{NR}=$ nuclear ring; $\mathrm{NS}=$ nuclear spiral; $\mathrm{OPG}=$ evidence for off-plane gas or dust, dusty = too dust-obscured to determine presence or absence of central structures. Derivation of disk inclination $i$ is described in the text. Rotation $(+=$ counter-clockwise,$-=$ clockwise, $\cdots=$ unknown) assumes that spiral arms trail, if any can be seen. "Size" is the radial extent of a feature in arc seconds, either semi-major axis or (for outer disks) $R_{25}$. "[Outer Disk]" indicates that a distinct outer disk cannot be identified; we list $R_{25}$ and best estimates for overall disk orientation.

${ }^{a}$ No reliable inclination can be determined for this galaxy. 


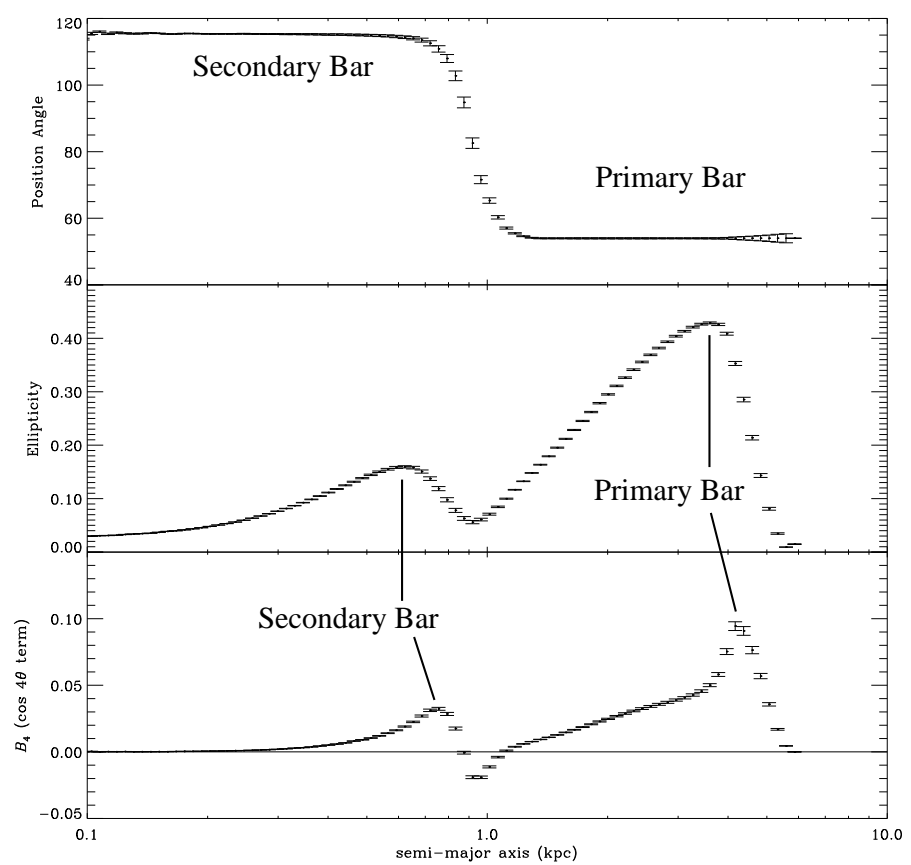

FIG. 1.- Ellipse fits to a face-on model double-bar galaxy, consisting of disk, bulge, and two Ferrers bars (Model 2 from Maciejewski 1998).

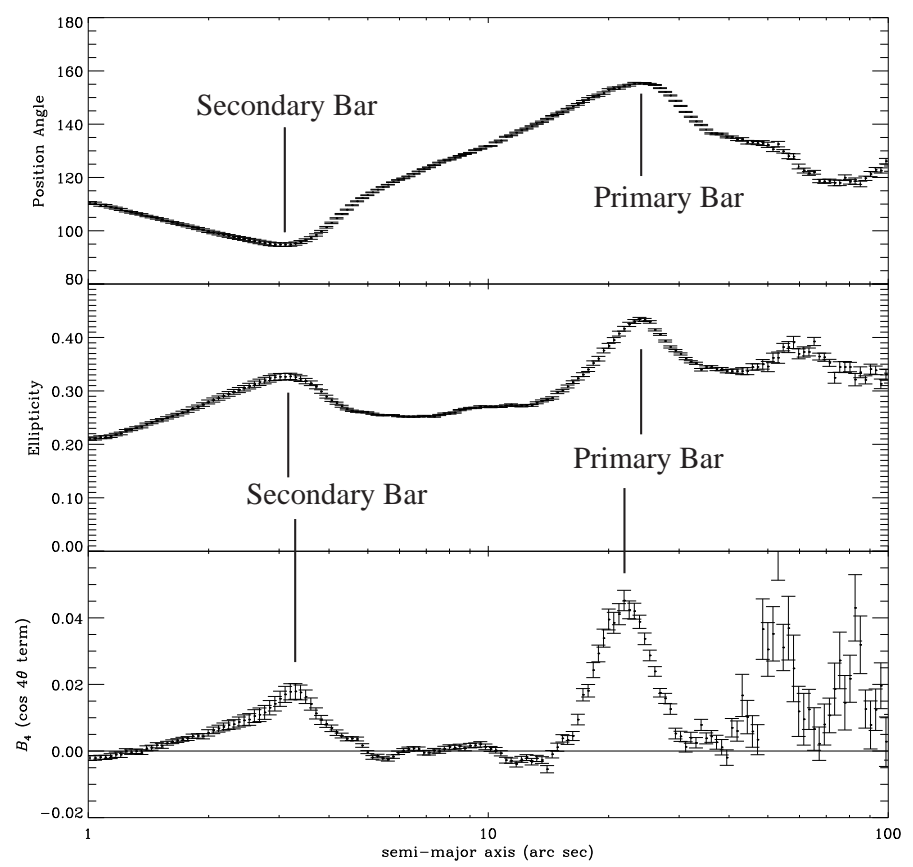

FIG. 2.- Ellipse fits to WIYN $R$-band image of the double-barred galaxy NGC 2950; see Figure 16 for other data on this galaxy.

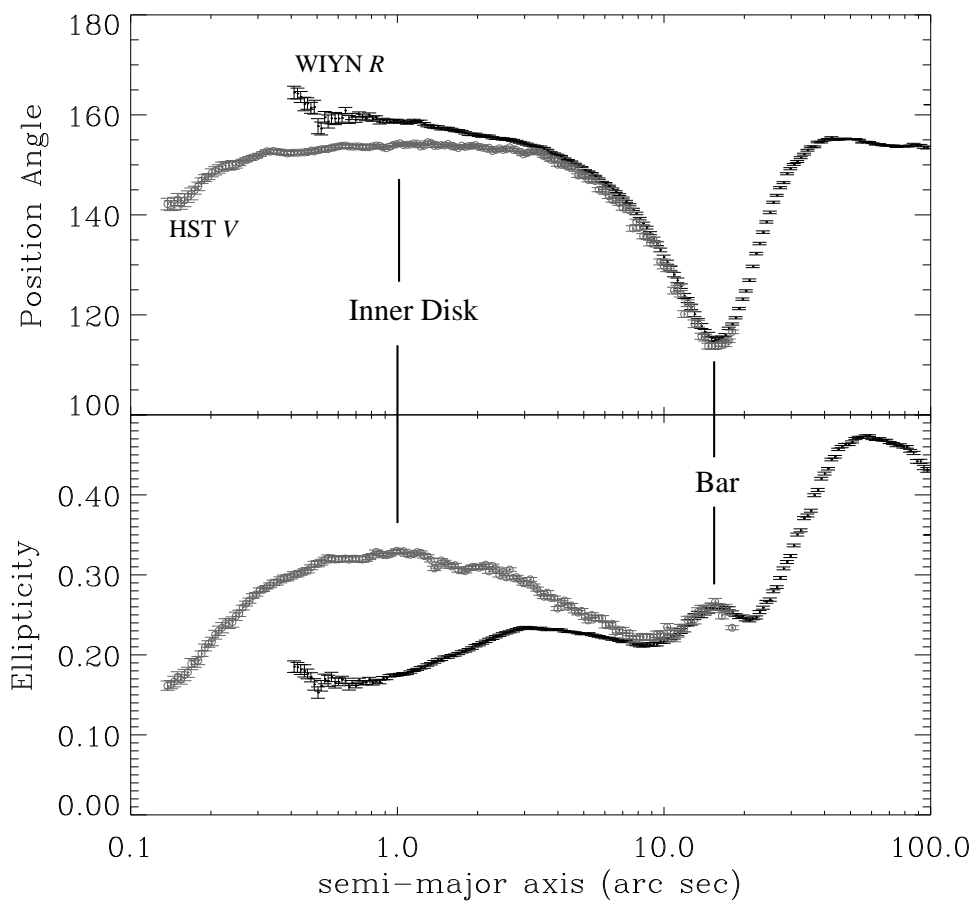

FIG. 3.- Ellipse fits to WIYN $R$-band and WFPC2 F606W (gray) images of the inner-disk galaxy NGC 3412; see Figure 20 for other data on this galaxy. 

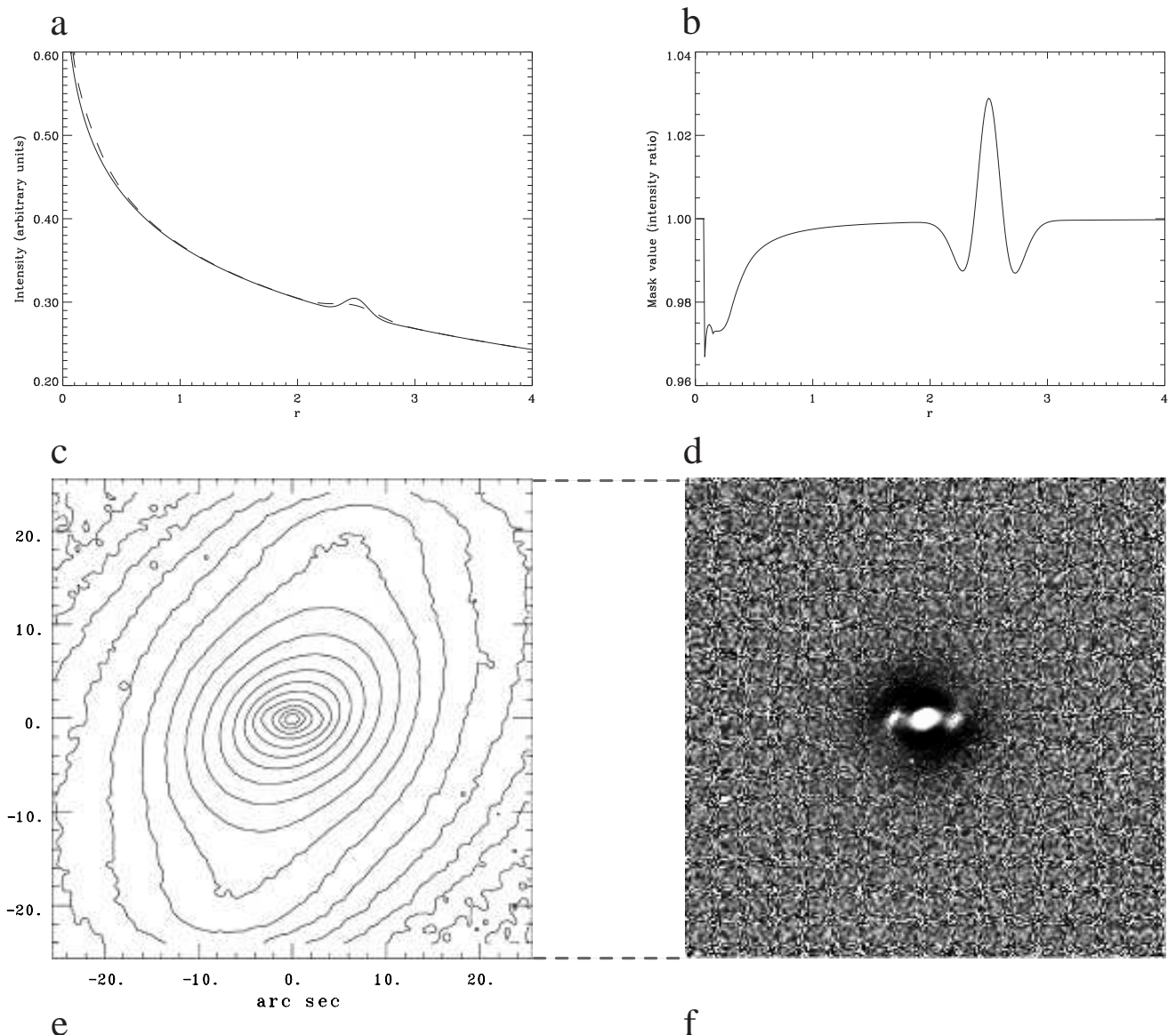

d

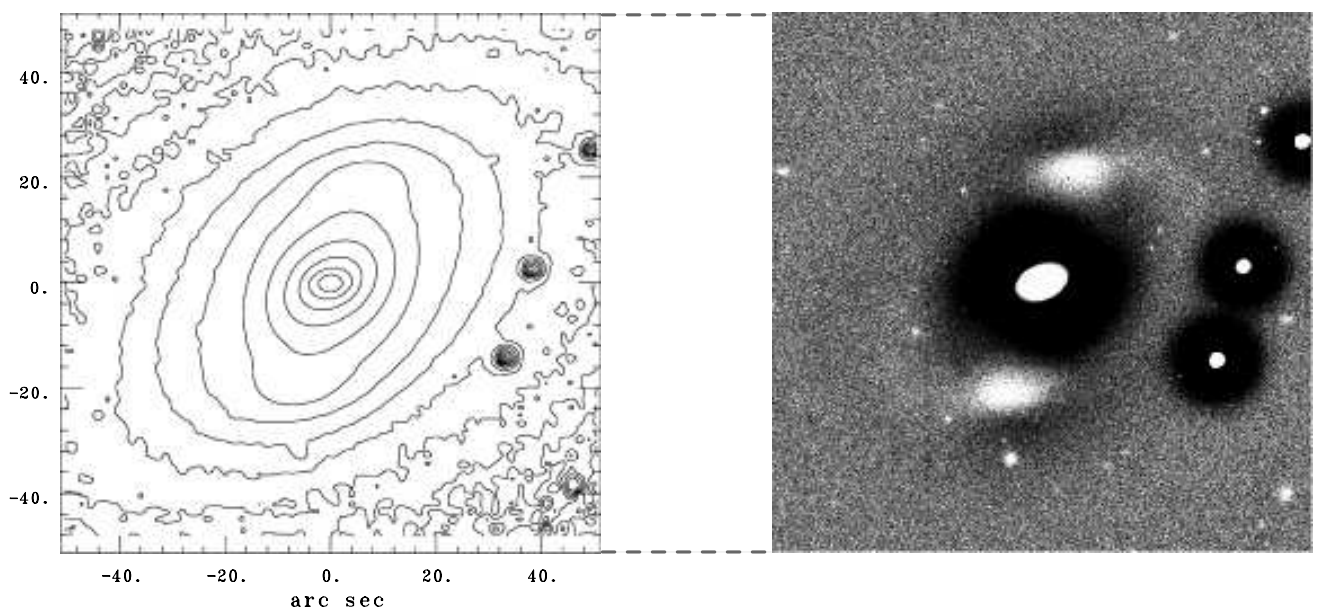

FIG. 4. - Examples illustrating the use of unsharp masks. a 1-D $r^{1 / 4}$ intensity profile with narrow Gaussian added at $r=2.5$ (solid) along with Gaussian-smoothed version (dashed); b 1-D unsharp mask produced by dividing original profile by smoothed profile. $\mathbf{c} R$-band contours of double-barred galaxy NGC 2950; d unsharp mask $(\sigma=5)$ of the same image, showing secondary bar. e Larger-scale $R$-band contours of the same galaxy; $\mathbf{f}$ unsharp mask $(\sigma=20)$, showing primary bar. 

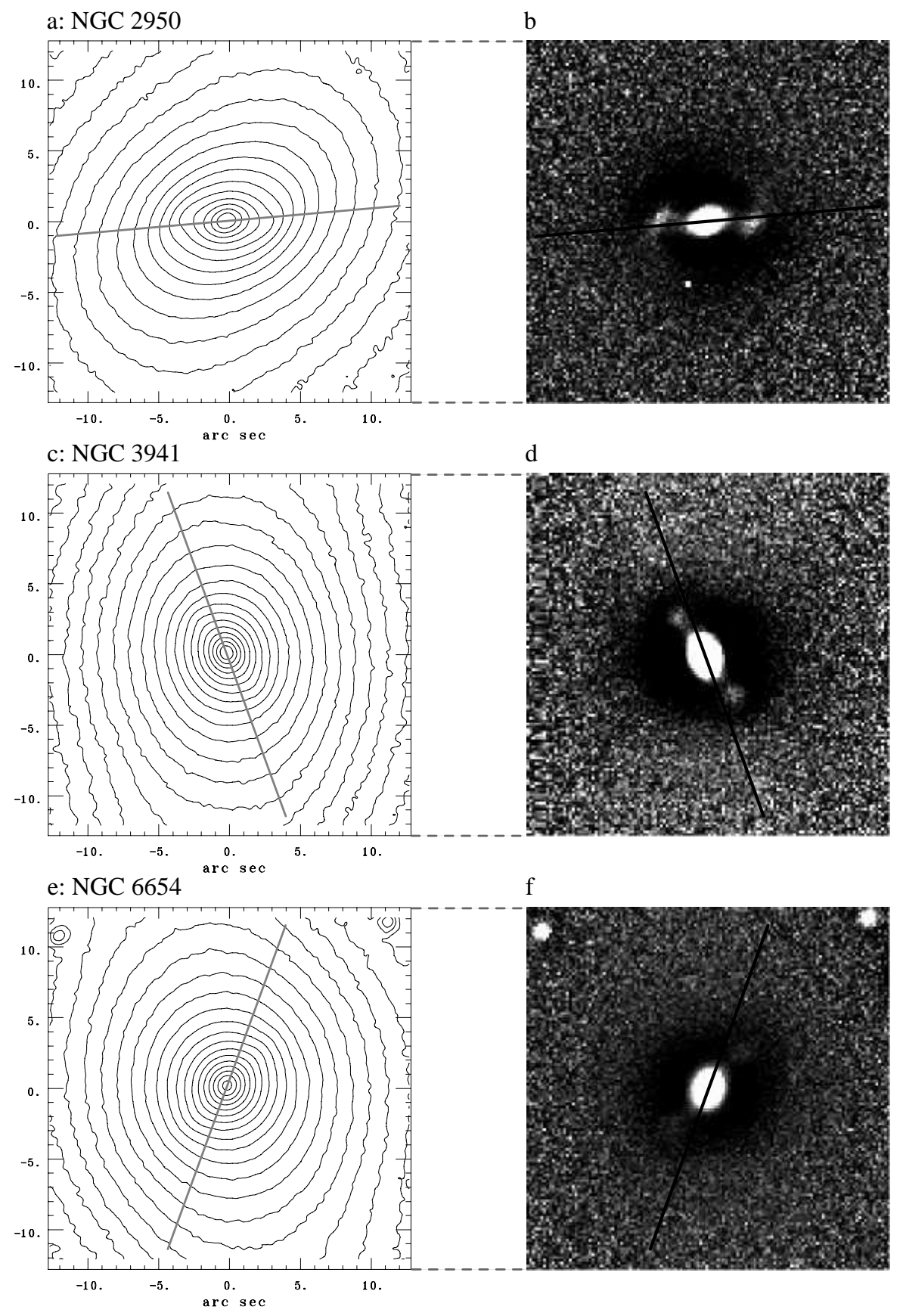

FIG. 5.- Examples of differences between position angles of secondary bars as given by ellipse fits (diagonal lines) versus actual isophotes (left) and unsharp masks $(\sigma=5$, right). 


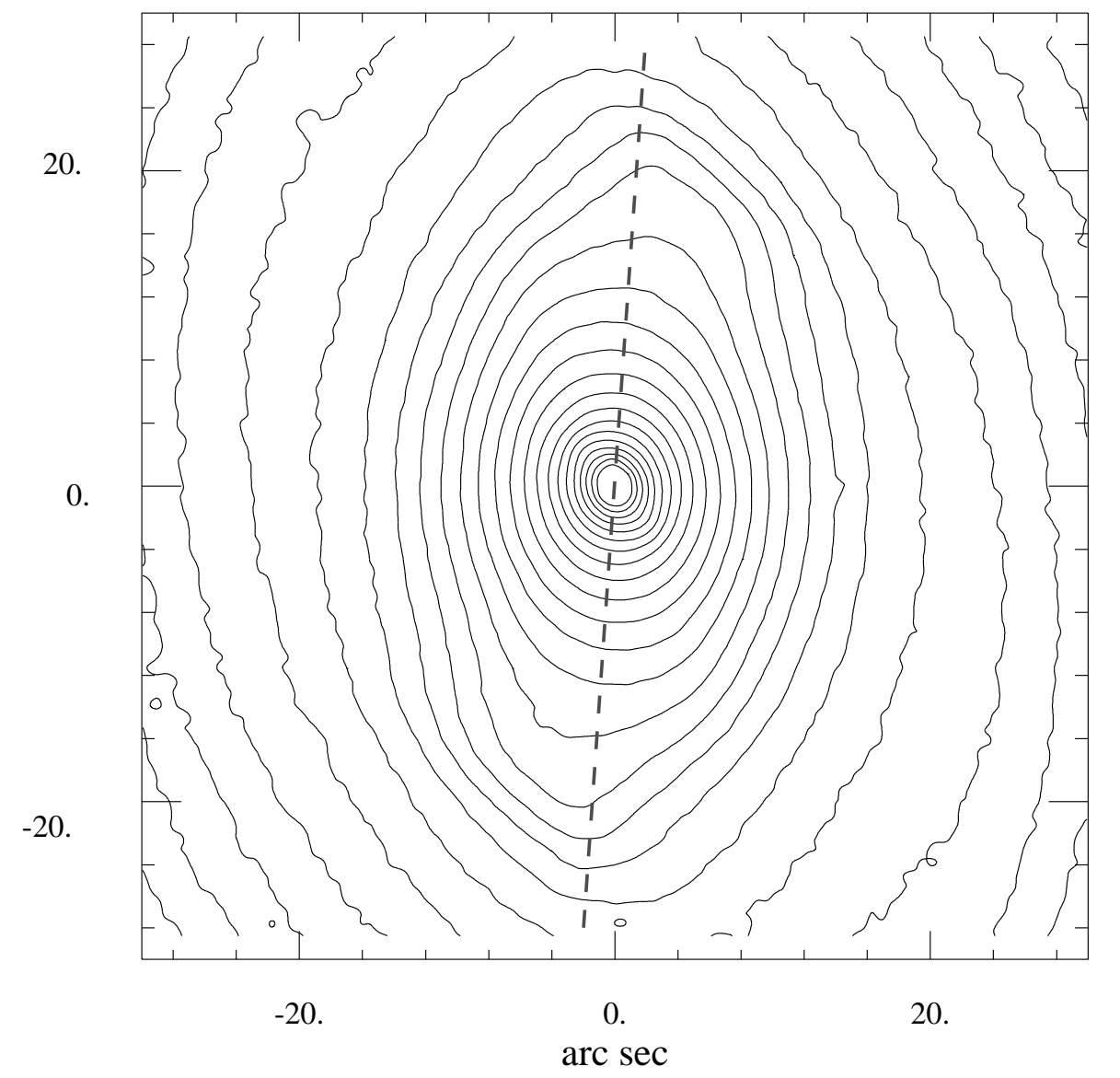

FIG. 6. - $R$-band image of the double-barred galaxy NGC 3941, showing the outer bar. The dashed line shows the position angle of the bar as derived from ellipse fits - clearly misaligned with the bar. As with inner bars (Figure 5), the position angles derived from ellipse fits must be treated with skepticism. The ellipse fits can be found in Figure 24. 

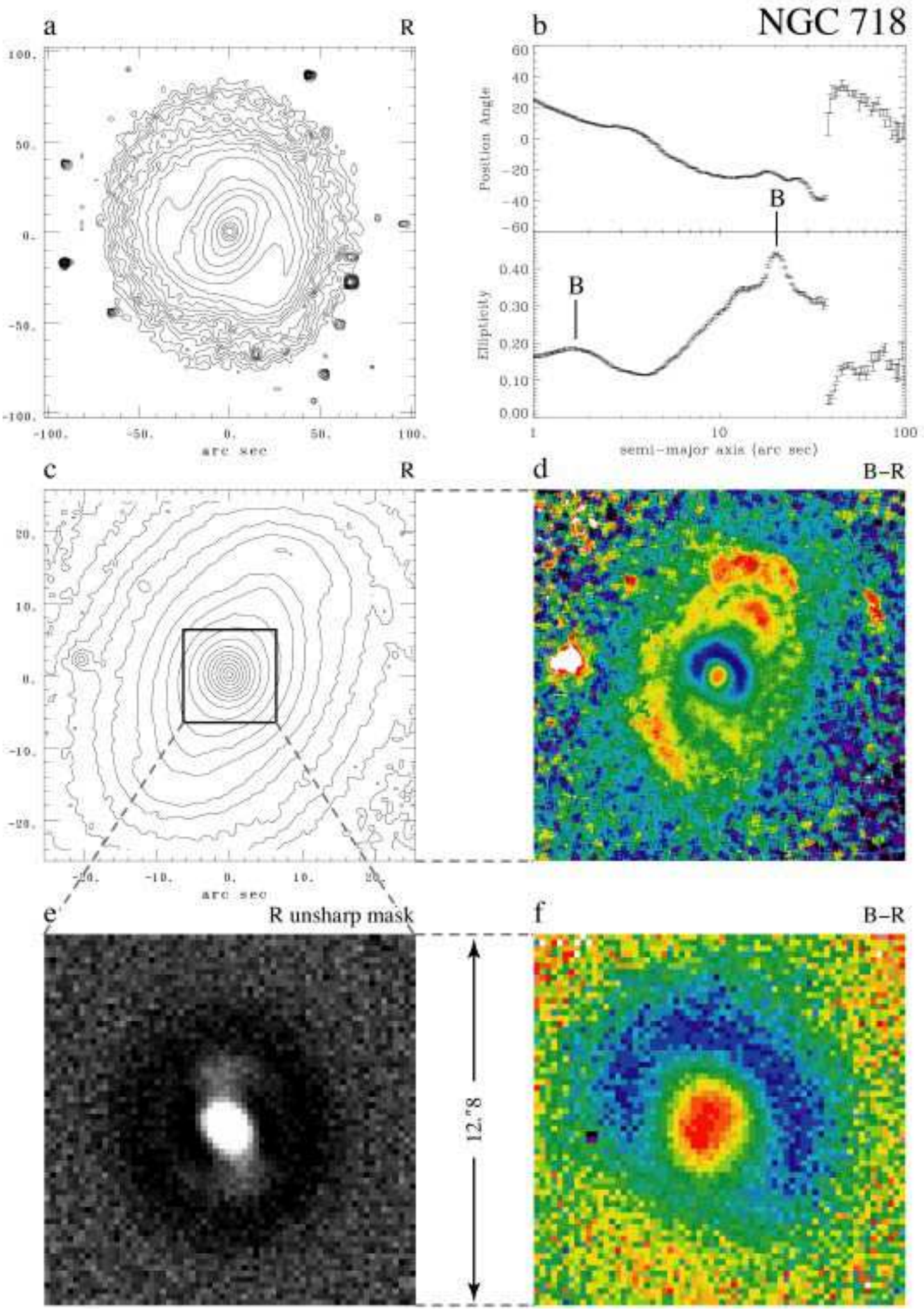

FIG. 7.- NGC 718: $R$-band isophotes on two scales (a, c); ellipse fits (b, WIYN $R$ only); $B-R$ color maps (d, median smoothing width $w=5$ pixels, color range $=0.85$ mag; $\mathrm{f}$, unsmoothed, color range $=0.28$ mag between ring and galaxy nucleus); $R$-band unsharp mask (e, Gaussian $\sigma=2$ pixels). 


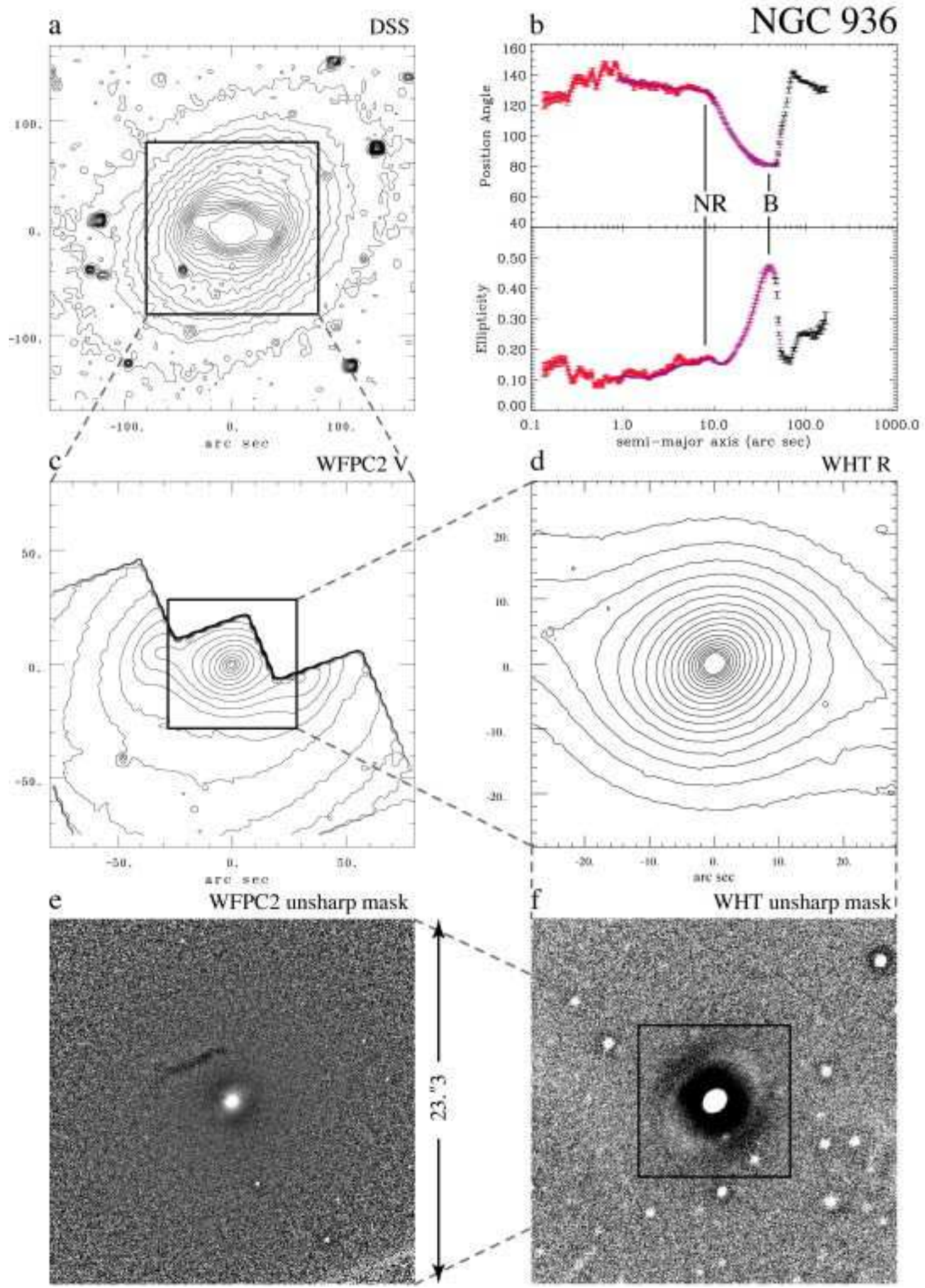

FIG. 8.- NGC 936: large-scale DSS isophotes (a); ellipse fits (b, PC2 F555W [red], WHT R [purple], DSS [black]); WFPC2 mosaic (c); WHT $R$-band image (d), unsharp mask of PC2 image $(\mathrm{e}, \sigma=10)$; the bright line at the lower right edge of the unsharp mask image is the edge of the PC2 chip; unsharp mask of WHT $R$-band image (f, $\sigma=10$ ). 

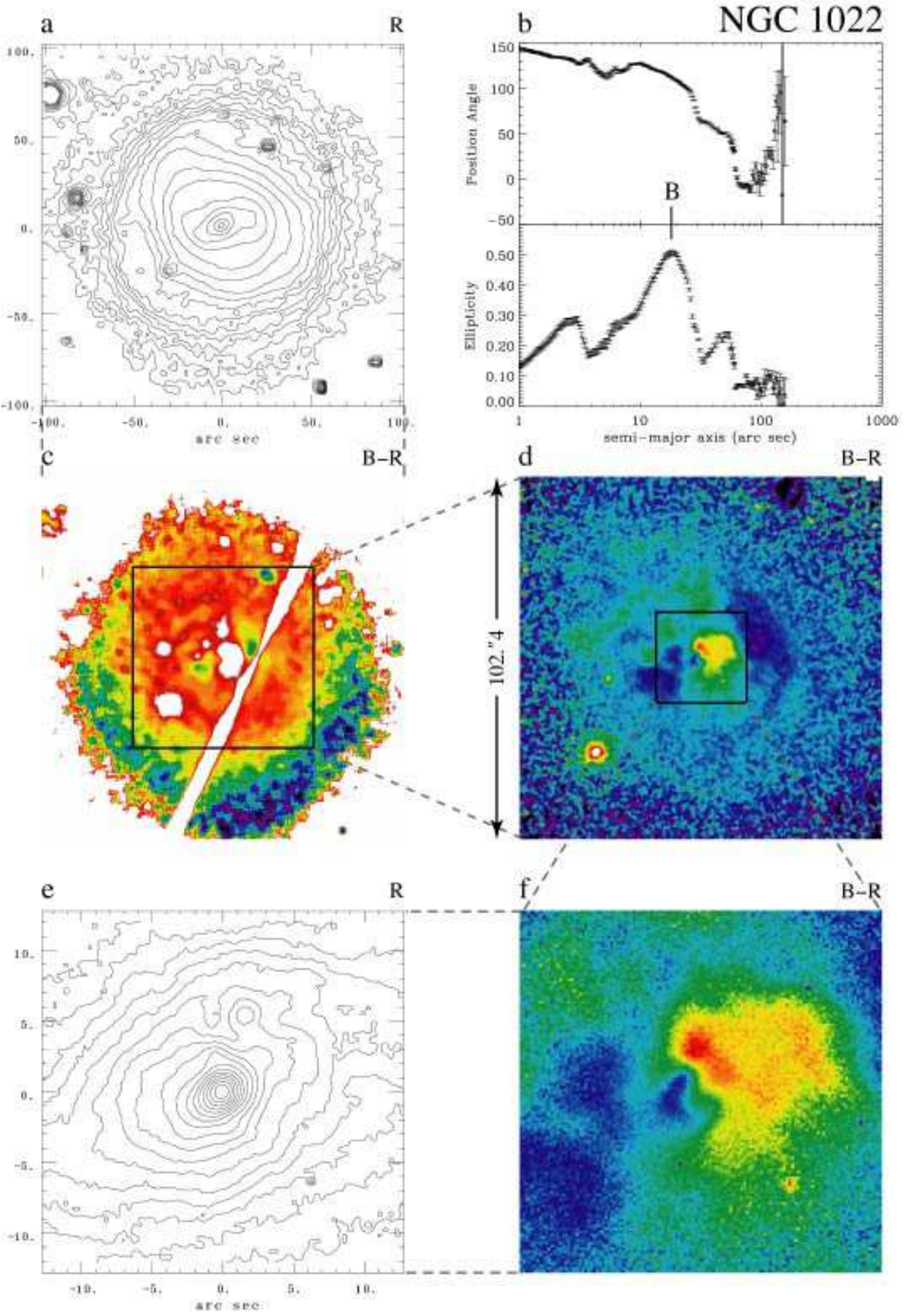

FIG. 9. - NGC 1022: $R$-band isophotes on two scales (a, e); ellipse fits (b, WIYN $R$ only); $B-R$ color maps (c, $w=23$, color range $\approx 1.40$ mag [excluding satellite.meteor trail); $\mathrm{d}, w=5$, color range $=1.53 \mathrm{mag} ; \mathrm{f}$, unsmoothed, color range $=0.90 \mathrm{mag}$ ). The red diagonal streak in panel c is due to a bright satellite or meteor trail during the long $R$-band exposure; the color maps in panels $\mathrm{d}$ and $\mathrm{f}$ were made with the unmarred short $R$-band exposure. 


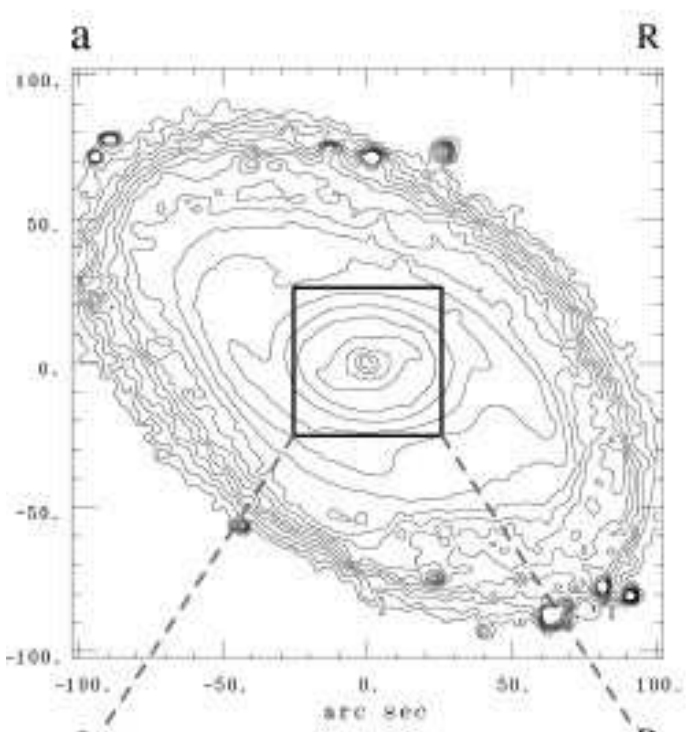

$\mathrm{R}$
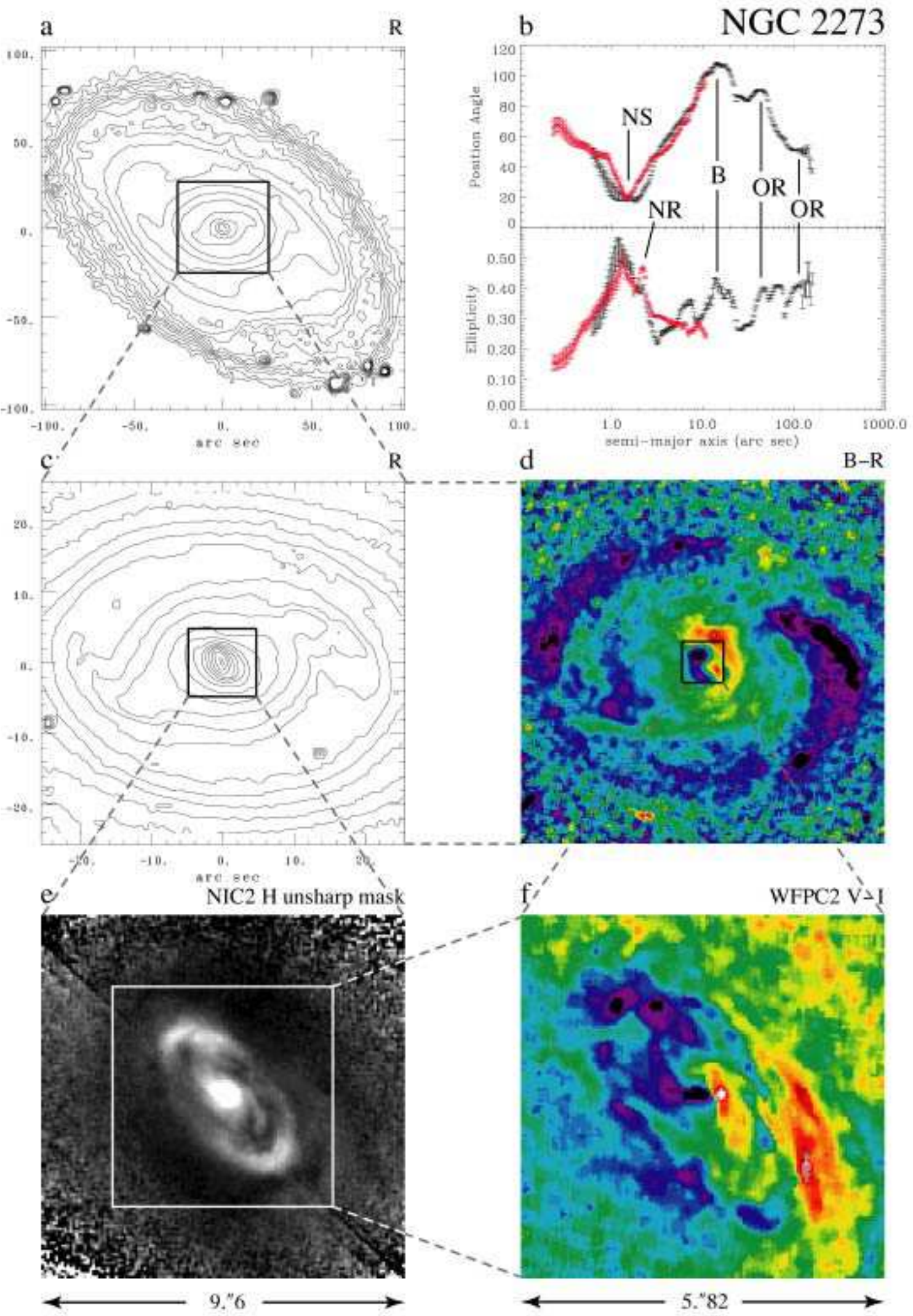

FIG. 10. - NGC 2273: $R$-band isophotes on two scales (a, c); ellipse fits (b, WIYN $R$ and NICMOS2 F160W [red]); $B-R$ color map (d, $w=5$, color range $=0.89 \mathrm{mag}$ ); PC2 $V-I[\mathrm{~F} 606 \mathrm{~W}-\mathrm{F} 791 \mathrm{~W}]$ color map (f, $w=5$, color range $=1.15$ mag); unsharp mask of NICMOS2 F160W image $(\mathrm{e}, \sigma=10)$. 

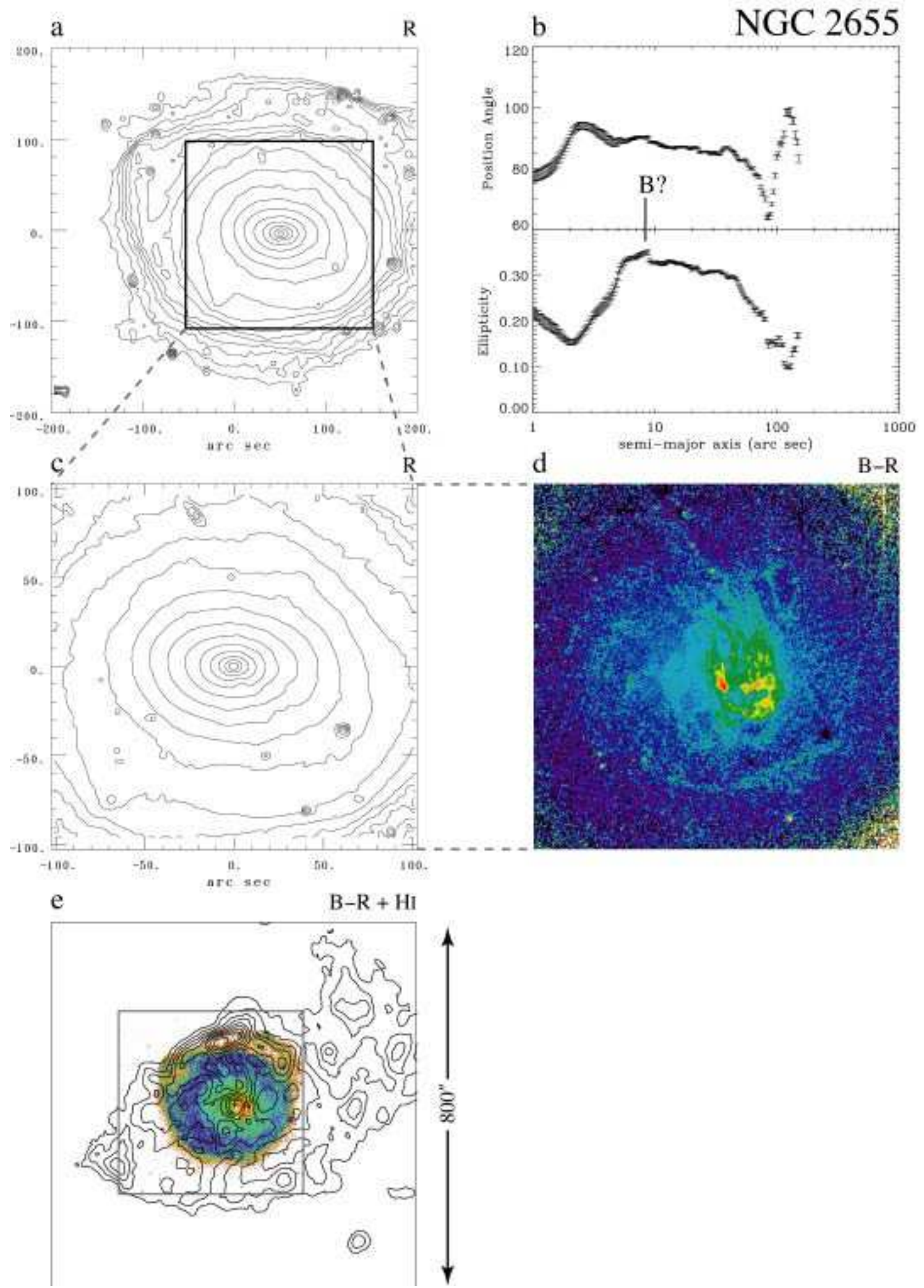

FIG. 11. - NGC 2655: $R$-band isophotes on two scales (a, c); ellipse fits (b, WIYN $R$ ); $B-R$ color map $(\mathrm{d}, w=5$, color range $\approx 1.2$ mag; $\mathrm{e}, w=11$, color range $\approx 1 \mathrm{mag}$, with H I radio contours). The contour map in panel a is the entire WIYN CCD; the H I contours in panel $\mathrm{e}$ is from van Moorsel et al. (2002). 


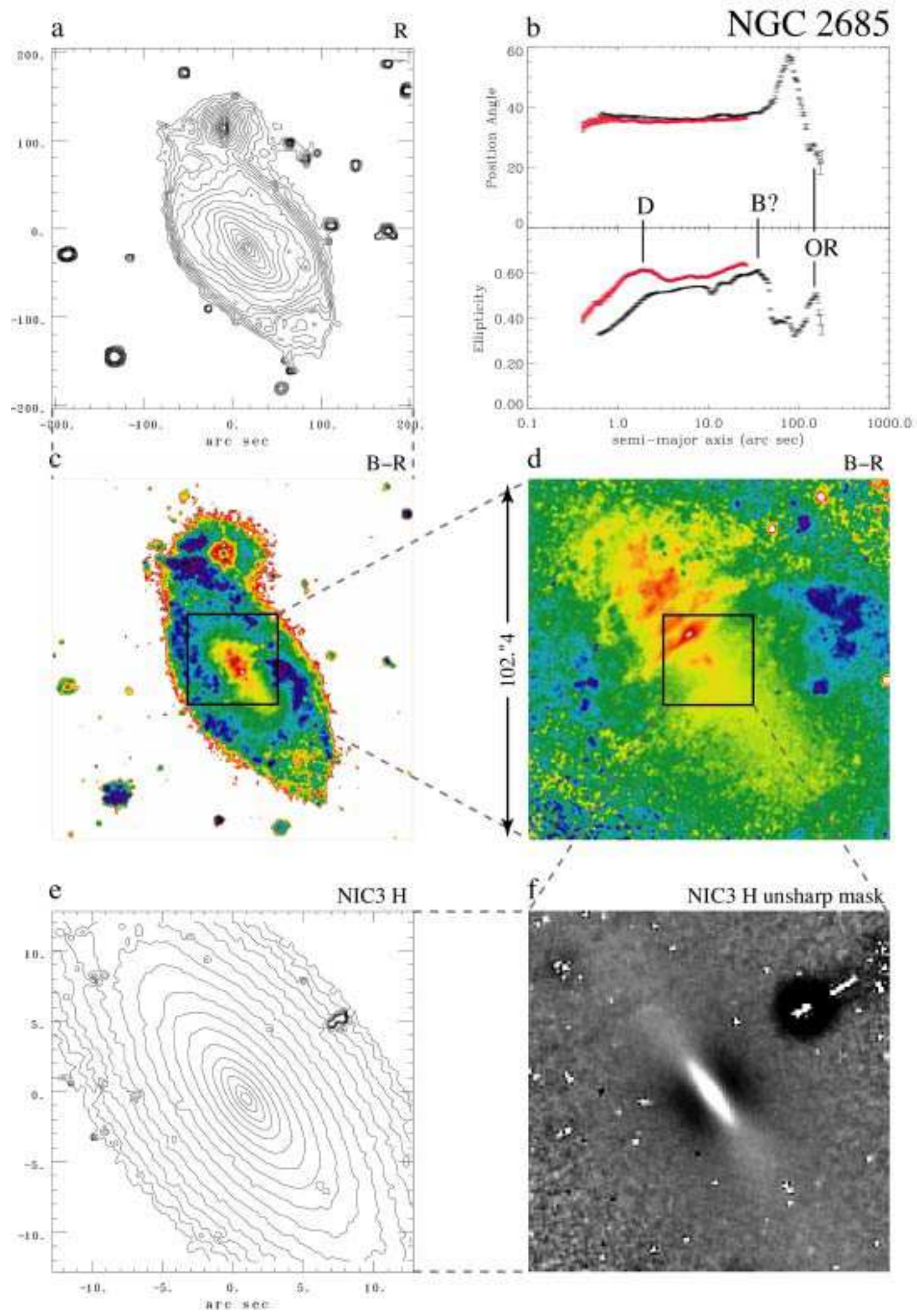

FIG. 12.- NGC 2685: $R$-band isophotes (a); ellipse fits (b, WIYN $R$ and NICMOS3 F160W [red]); $B-R$ color maps (c, $w=23$, color range $\approx 1.0 \mathrm{mag} ; \mathrm{d}$, unsmoothed, color range $=1.48 \mathrm{mag}$ ); NICMOS3 F160W isophotes (e) and unsharp mask (f, $\sigma=5)$. The contour map in panel a and the color map in panel c are the entire WIYN CCD. 

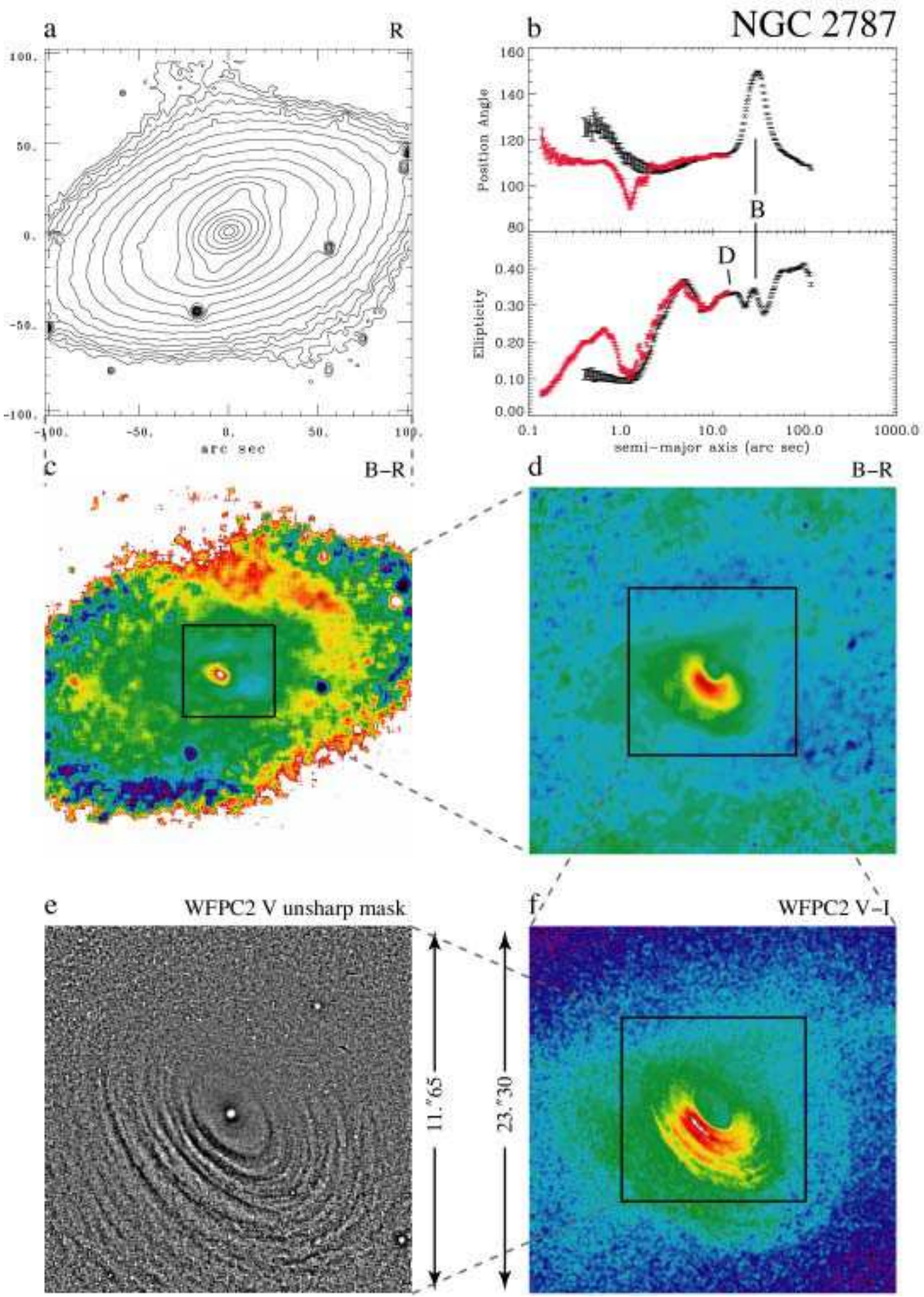

Fig. 13. - NGC 2787: $R$-band isophotes (a); ellipse fits (b, WIYN $R$ and PC2 F814W [red]); $B-R$ color map (c, $w=23$, color range $=$ $0.65 \mathrm{mag}$ ); PC2 $V-I[\mathrm{~F} 555 \mathrm{~W}-\mathrm{F} 814 \mathrm{~W}]$ color map (f, $w=5$, color range $=0.80 \mathrm{mag}$ ); unsharp mask of F555W PC2 image $(\mathrm{e}, \sigma=5)$. The extensions on the NNE and SSW edges of the disk in panel a are due to scattered light from an extremely bright star just off the edge of the CCD. 

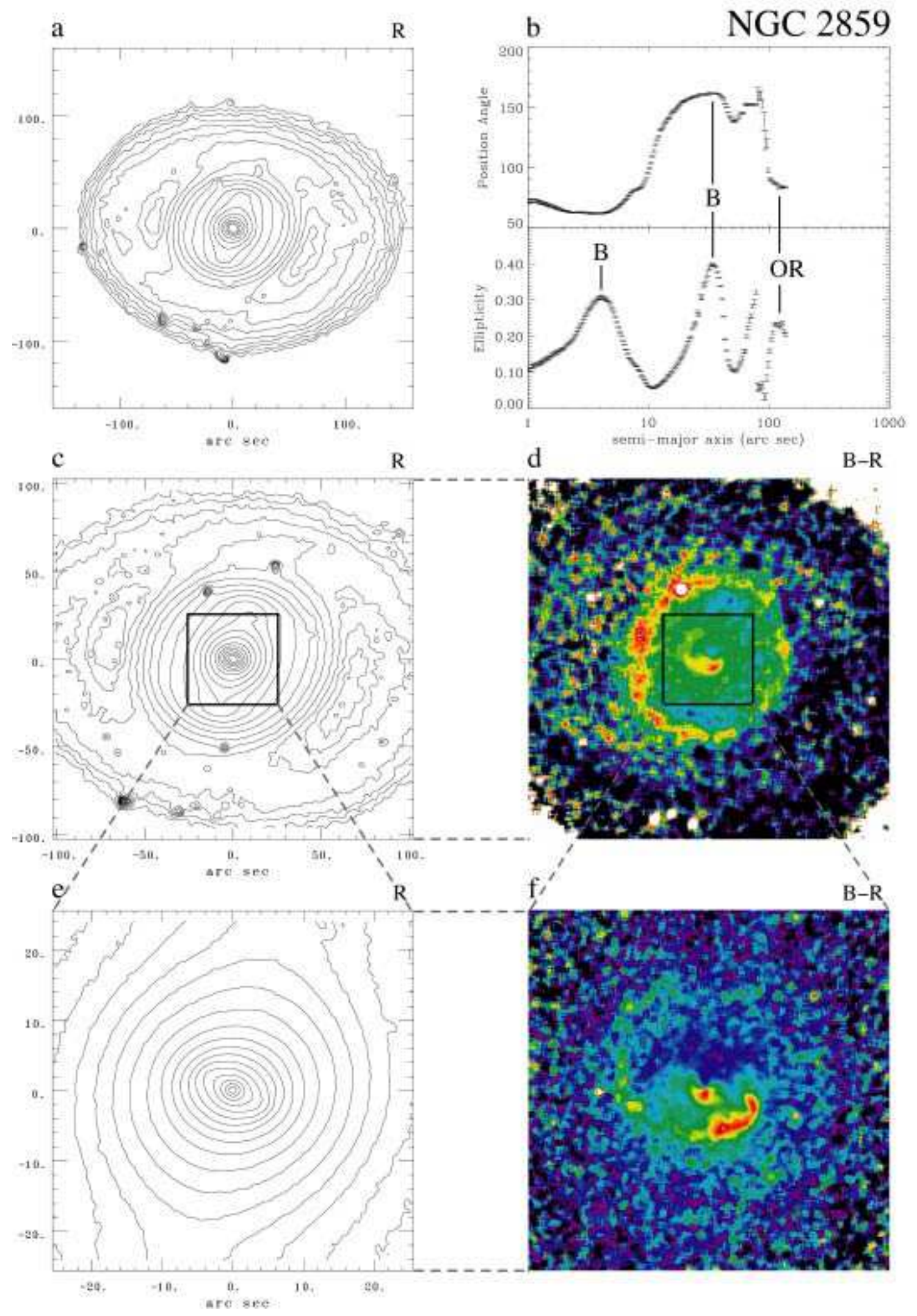

FIG. 14. - NGC 2859: $R$-band isophotes on various scales (a, c, e); ellipse fits (b, WIYN $R$ ); $B-R$ color maps $(\mathrm{d}, w=23$, color range $=$ $0.54 \mathrm{mag}$; f, $w=5$, color range $=0.27 \mathrm{mag}$ ). 

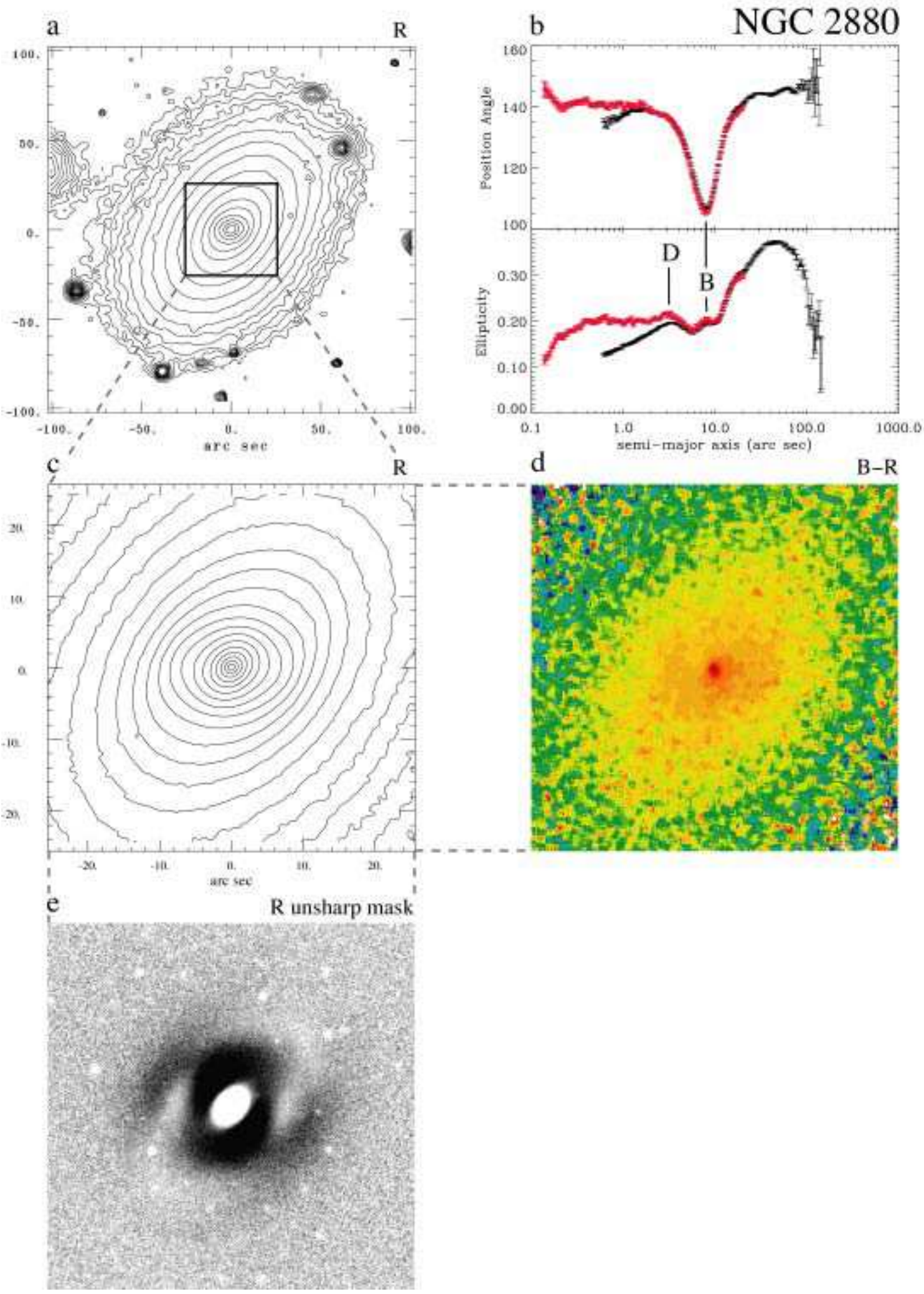

FIG. 15.- NGC 2880: $R$-band isophotes on two scales (a, c); ellipse fits (b, WIYN $R$ and WFPC2 F814W [red]); $B-R$ color map (d, $w=5$, color range $=0.39 \mathrm{mag}) ; \sigma=10$ unsharp mask of WIYN $R$-band image (e), showing the bar and spiral/ring just outside. 


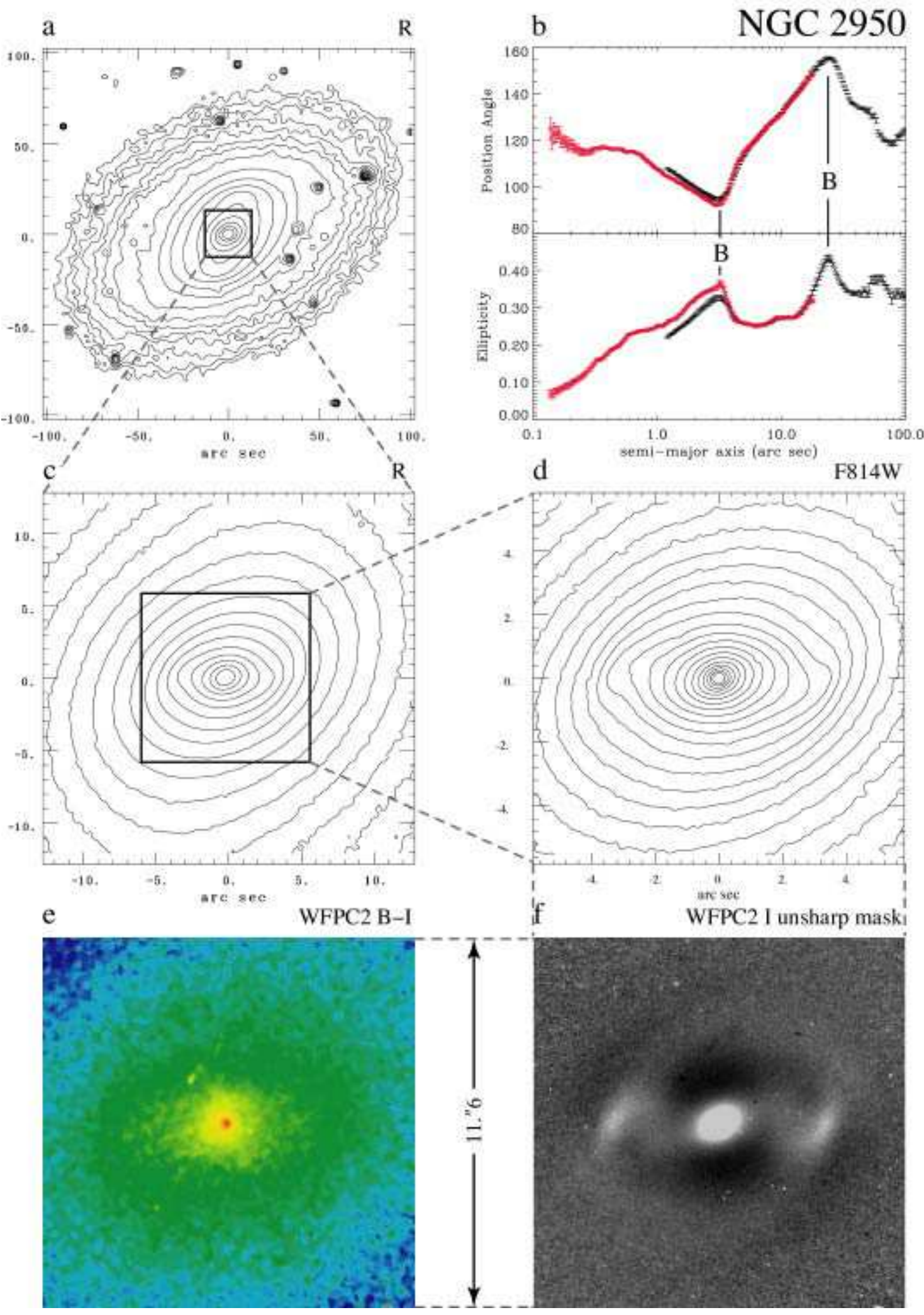

FIG. 16. - NGC 2950: $R$-band isophotes (a, c); ellipse fits (b, WIYN $R$ and WFPC2 F814W [red]); WFPC2 F814W isophotes (d); WFPC2 F450W $-\mathrm{F} 814 \mathrm{~W}$ colormap (e, $w=5$, color range $=0.38 \mathrm{mag}$ ); $\sigma=15$ unsharp mask of the WFPC2 F814W image 

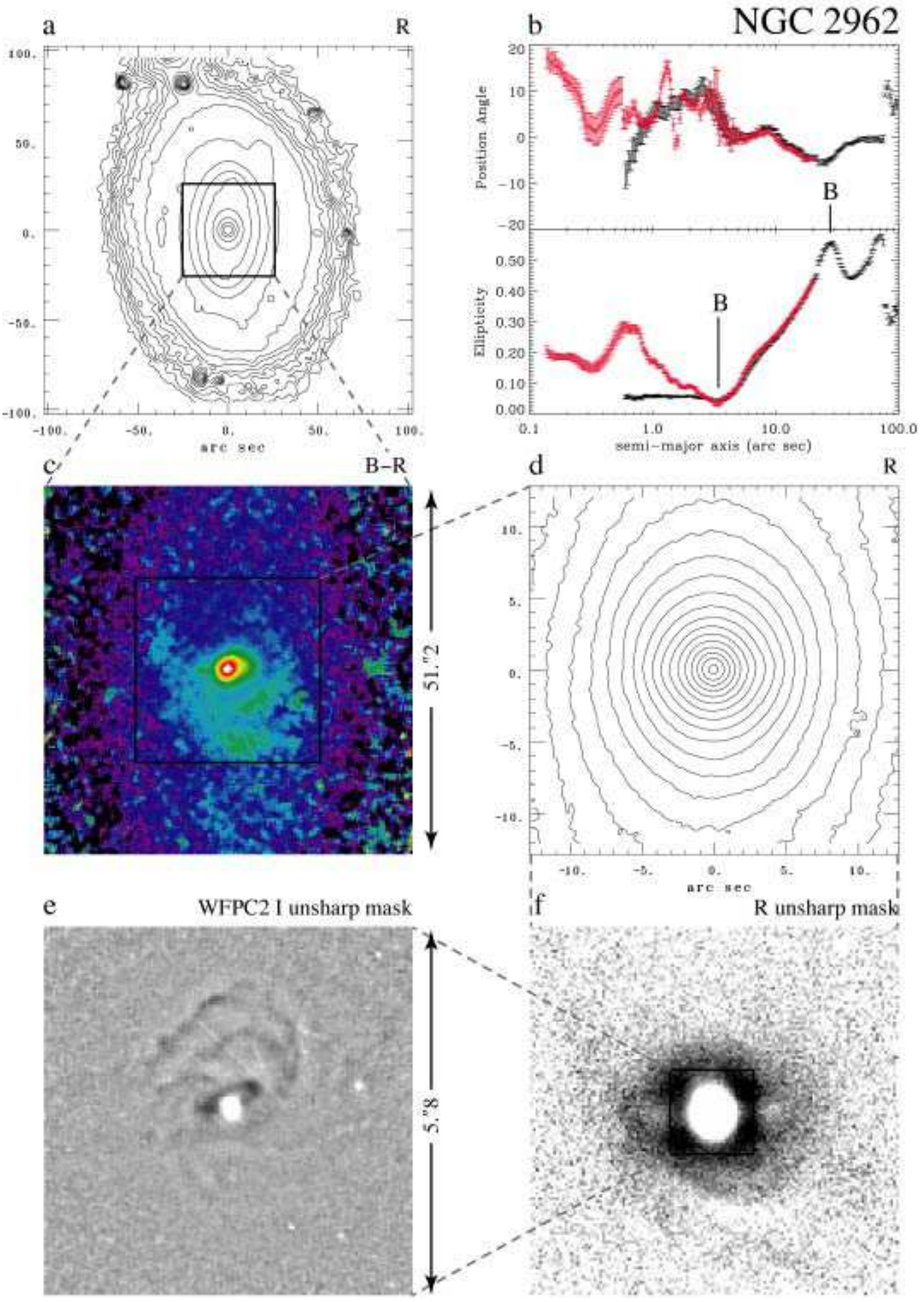

FIG. 17.- NGC 2962: $R$-band isophotes (a,d); ellipse fits (b, WIYN $R$ and WFPC2 F814W [red]); $B-R$ color map (c, $w=5$, color range $=0.52 \mathrm{mag}) ; \sigma=5$ unsharp mask of the $R$-band image (f), showing the secondary bar and dust lanes outside it; $\sigma=3$ unsharp mask of the WFPC2 F814W image. 

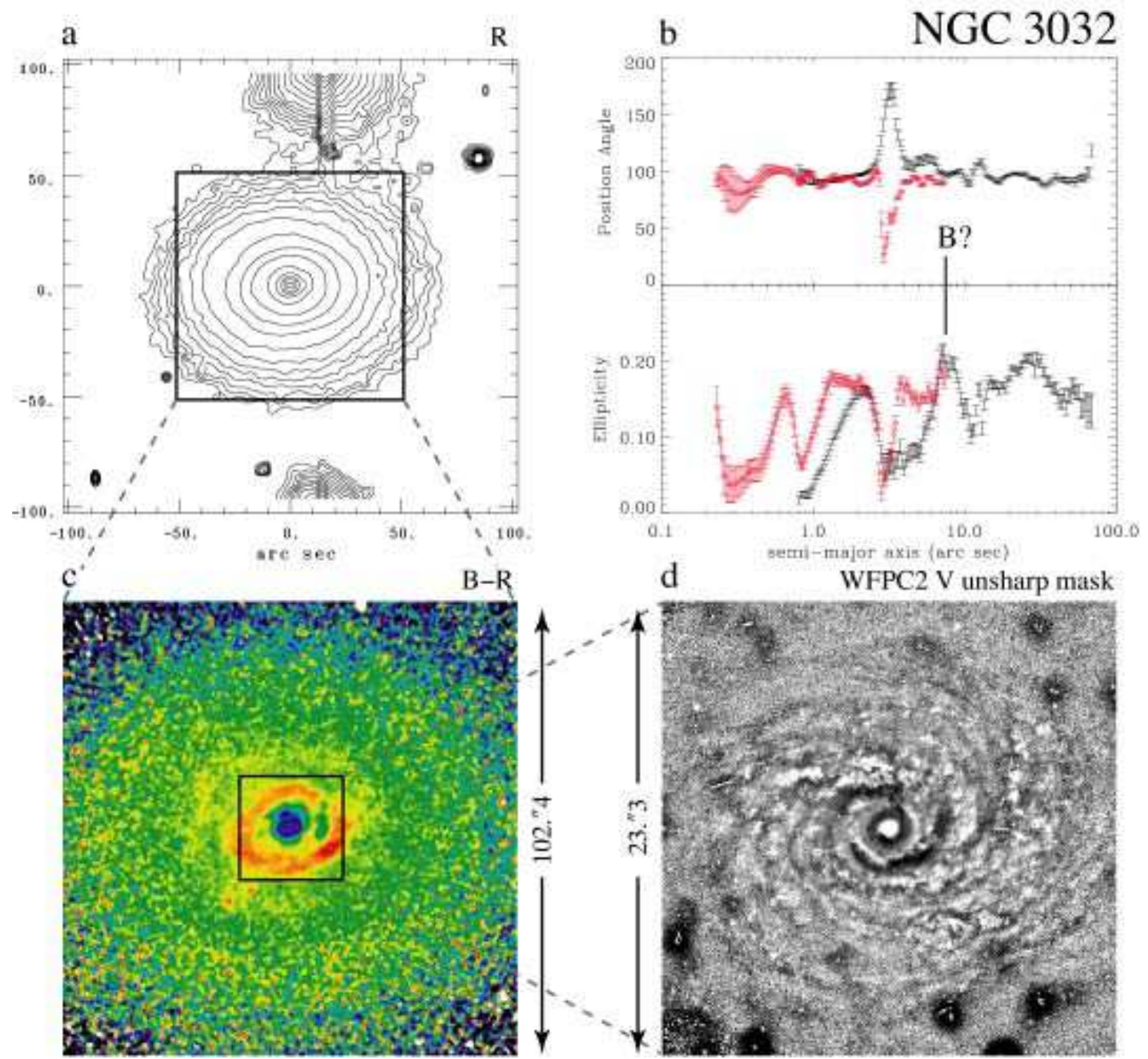

FIG. 18. - NGC 3032: $R$-band isophotes (a); ellipse fits (b, WIYN $R$ and NICMOS2 F160W [red]); WIYN $B-R$ color map (c, $w=5$, color range between dust ring and nuclear blue spots $=1.07 \mathrm{mag}$ ); and a $\sigma=10$ unsharp mask of the F606W PC2 image (d), showing the nuclear spiral. The two objects $\mathrm{N}$ and $\mathrm{S}$ of the galaxy in (a) are bright, saturated stars. Note that pixels in the central $0.6-0.7^{\prime \prime}$ of the PC2 image are saturated. 


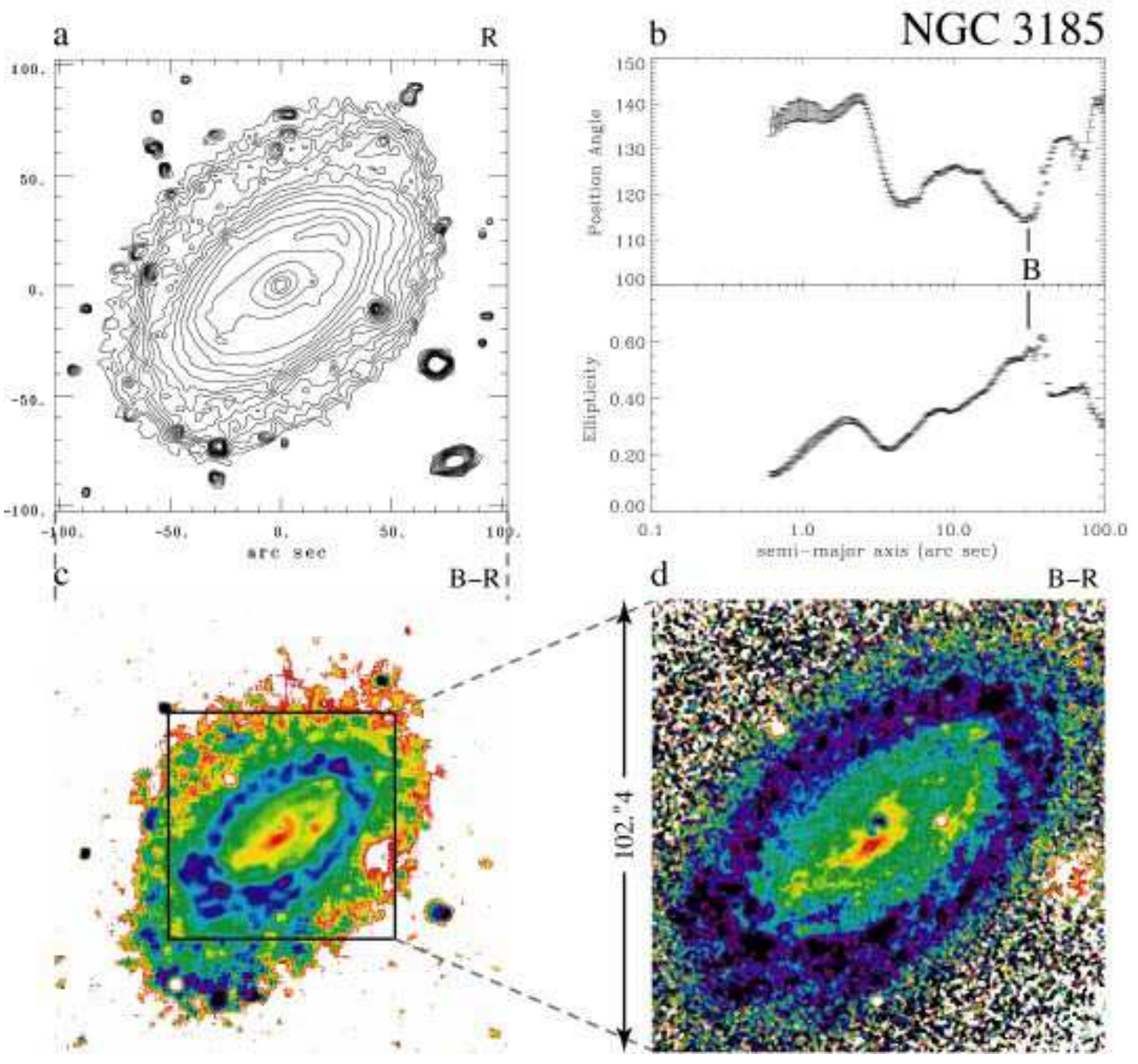

FIG. 19. - NGC 3185: large-scale $R$-band isophotes (a); ellipse fits (b, WIYN $R$ ); $B-R$ colors on the same scale (c, $w=23$, color range $=1.1 \mathrm{mag}$ ); and a closer view of the $B-R$ color map showing dust lanes in the bar and a possible nuclear dust ring (d, $w=5$, color range between inner ring and central dust lanes $=1.02 \mathrm{mag}$ ). 

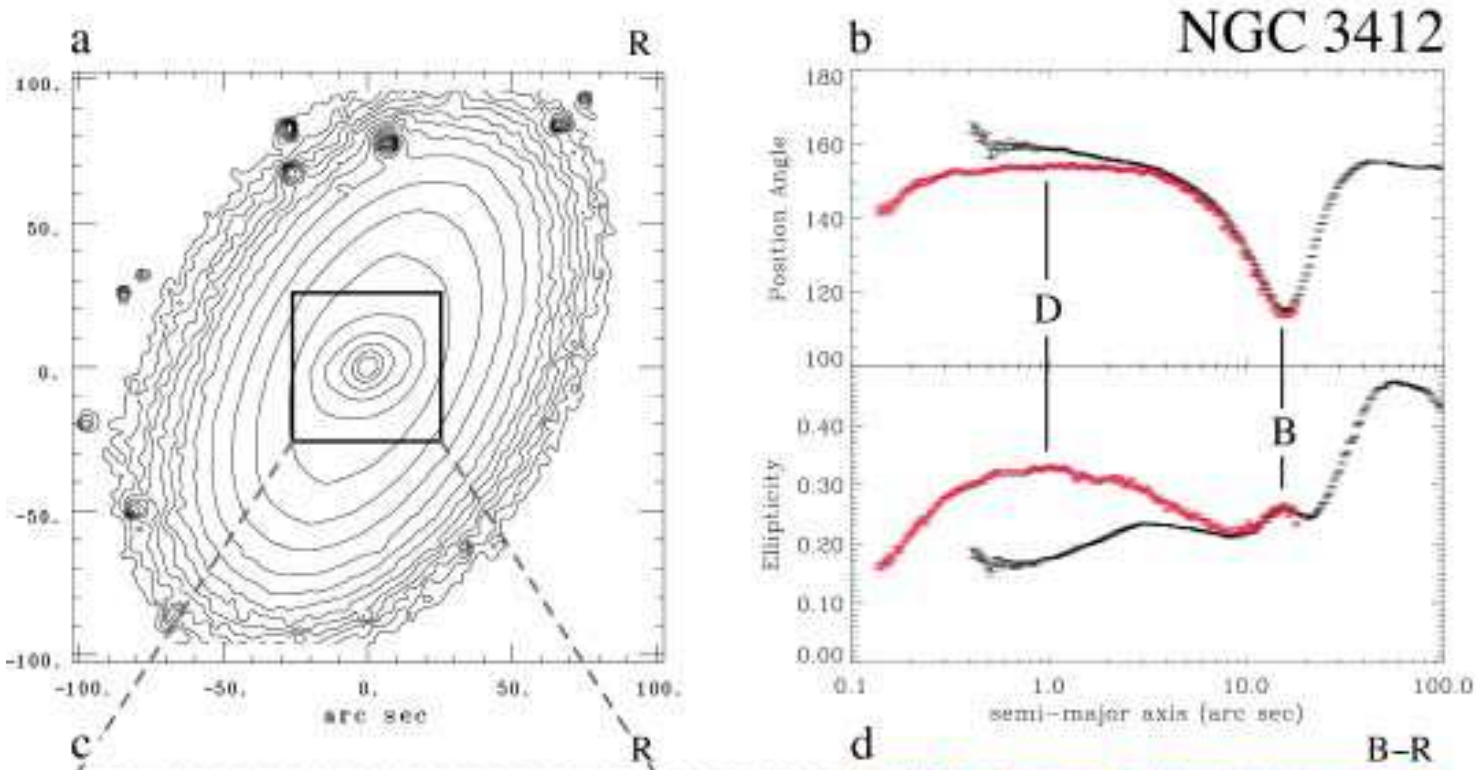

20.

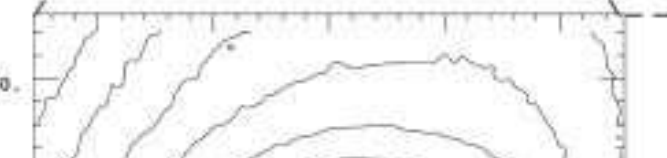

10.

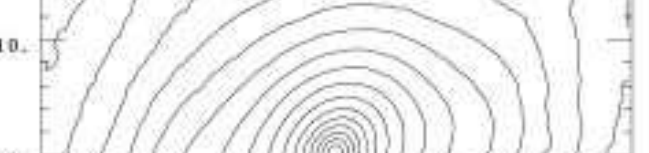

0.
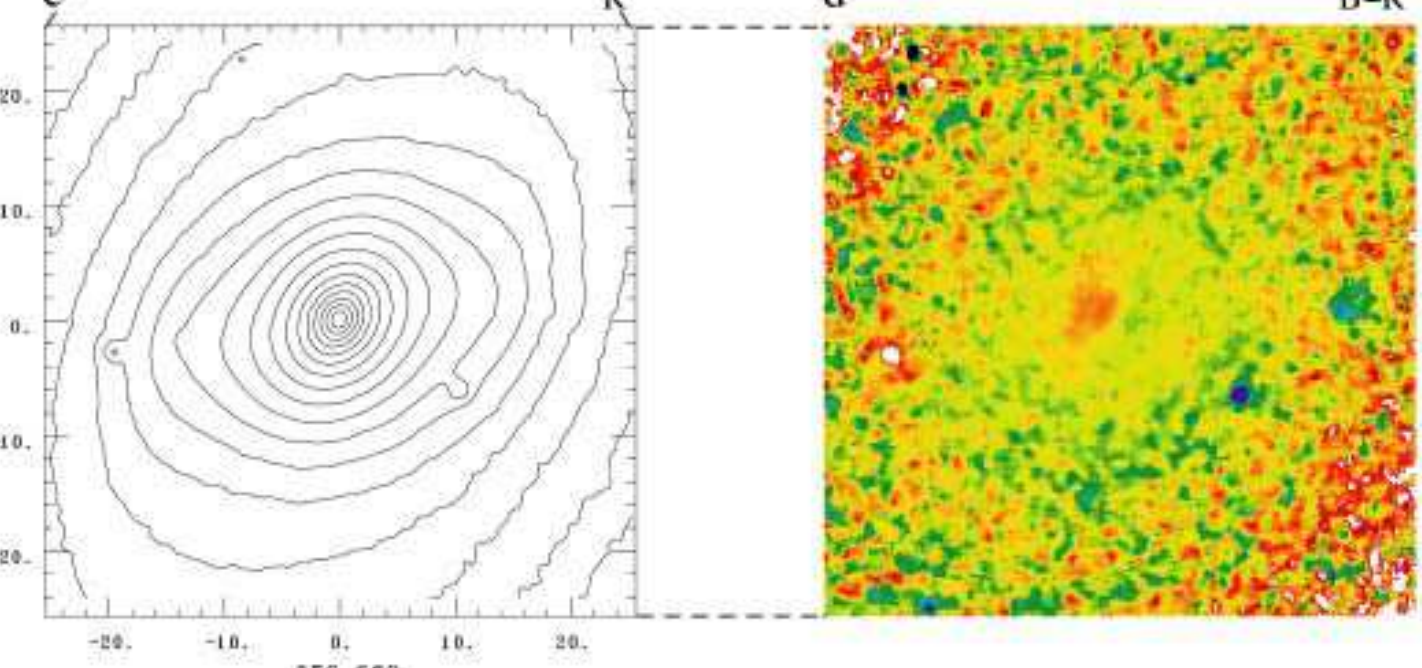

FIG. 20.- NGC 3412: large- and small-scale $R$-band isophotes (a, c); ellipse fits (b, WIYN $R$ and WFPC2 F606W); along with the $B-R$ color map $(\mathrm{d}, w=5$, color range $=0.27 \mathrm{mag})$. 


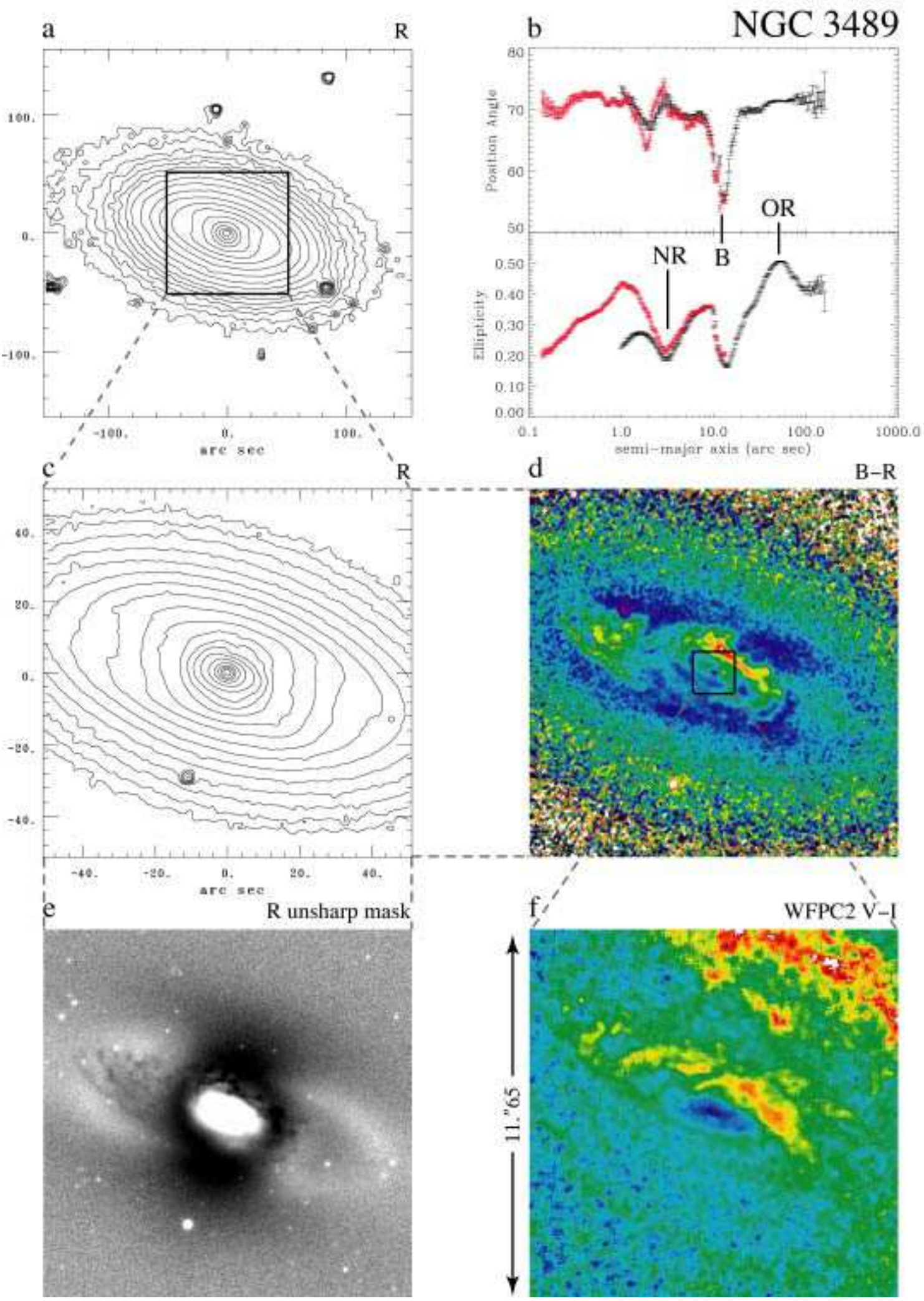

FIG. 21. - NGC 3489: large-scale (a) and medium-scale $R$-band isophotes (c); ellipse fits (b, WIYN $R$ and WFPC2 F814W [red]); $B-R$ color map $(\mathrm{d}, w=5$, color range $=0.57 \mathrm{mag}) ; \sigma=40$ unsharp mask of WIYN $R$-band image showing the outer ring, dust lanes, and the bar (e); HST F555W - F814W color map (f, $w=5$, color range $=0.80 \mathrm{mag}$ ) showing the nuclear dust ring and blue interior. 

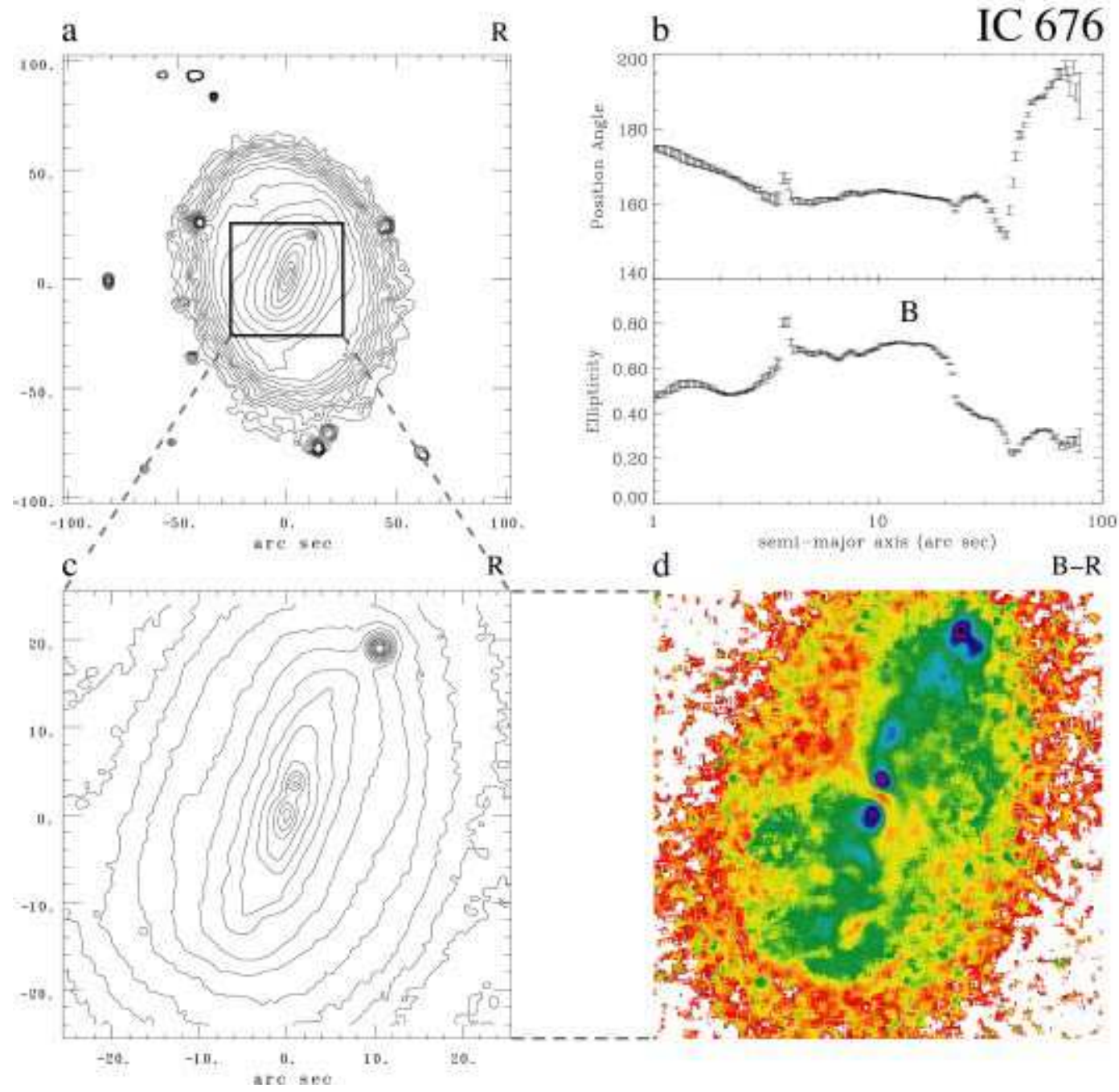

FIG. 22.- IC 676: large-scale $R$-band isophotes (a); ellipse fits (b, WIYN $R$ ); a close-up of the bar region (c), which shows the "binary nuclei"; and a $B-R$ color map of the same region (d, $w=5$, color range $=0.92 \mathrm{mag}$, excluding outer regions). The bright, blue object on the NW edge of the bar is a foreground star. 

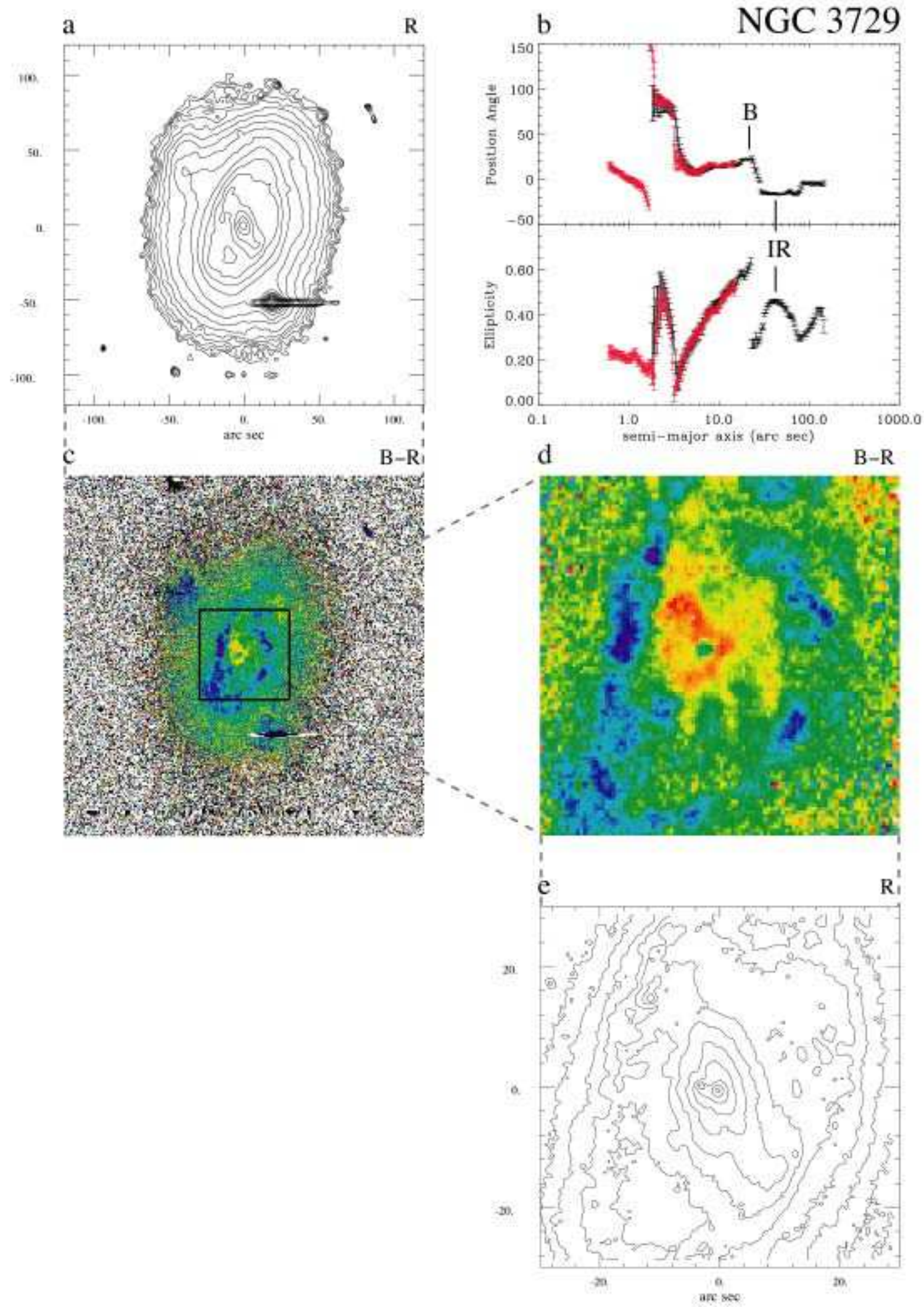

FIG. 23. - NGC 3729: large-scale Mauna Kea $R$-band contours (a); ellipse fits (b, Mauna Kea $R$ and WIYN $R$ [red]); $B-R$ color maps (c, no smoothing, color range $\approx 1.1 \mathrm{mag} ; \mathrm{d}$, no smoothing, color $\approx 0.8 \mathrm{mag}$ ); and a close-up of the bar region (e, $R$-band contours from WIYN image). 

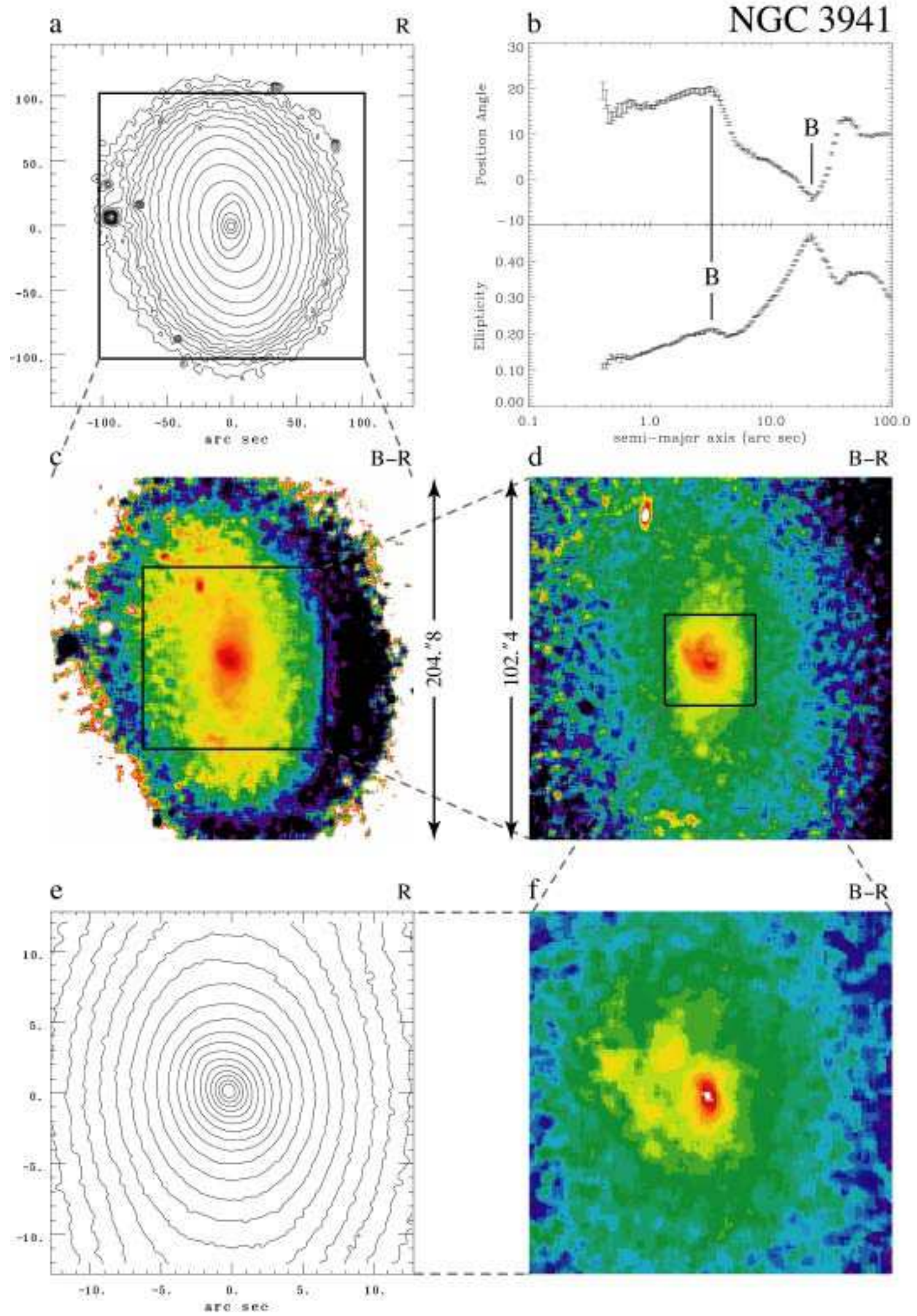

FIG. 24. - NGC 3941: large-scale $R$-band isophotes (a); ellipse fits (b, WIYN $R$ ); $B-R$ color maps at two scales (c, $w=23$, color range $=$ $0.51 \mathrm{mag} ; \mathrm{d}, w=11$, color range $=0.39 \mathrm{mag})$; small-scale isophotes $(\mathrm{e})$; and $B-R$ color map at the same scale $(\mathrm{f}, w=5$, color range $=0.51$ $\mathrm{mag})$. The apparent blue nucleus in (d) is an artifact of saturation in the $R$-band image; the shorter-exposure $R$-band image used in (f) was not saturated. 


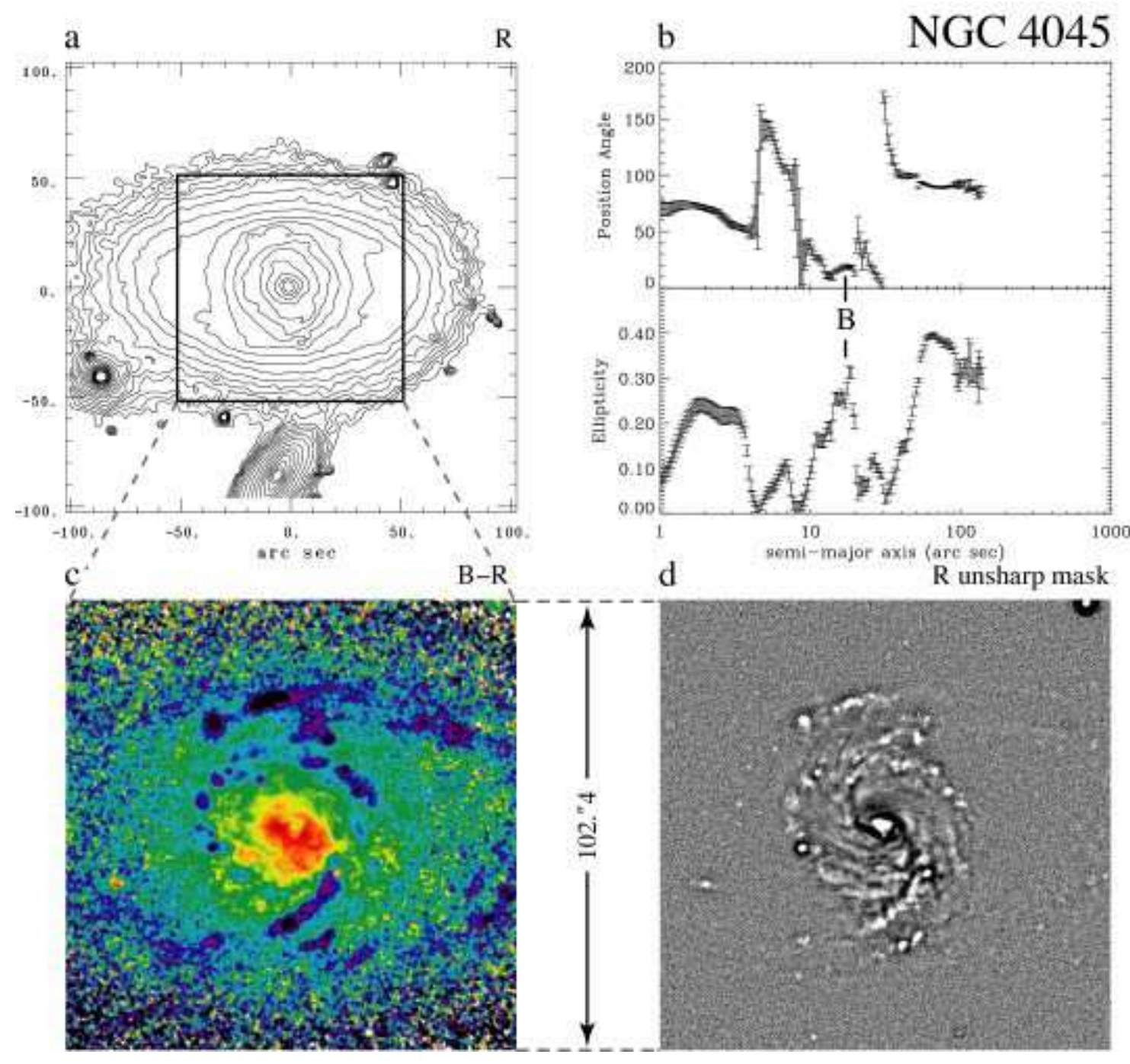

FIG. 25.- NGC 4045: $R$-band isophotes (a); ellipse fits (b, WIYN R); $B-R$ color map (c, $w=5$, color range $=1.13$ mag); and $\sigma=5$ unsharp mask of the $R$-band image, showing dust lanes and blue spiral arms. The disk galaxy immediately to the south, NGC $4045 \mathrm{~A}$, is at a redshift of $5040 \mathrm{~km} \mathrm{~s}^{-1}$ and so is not physically associated. 

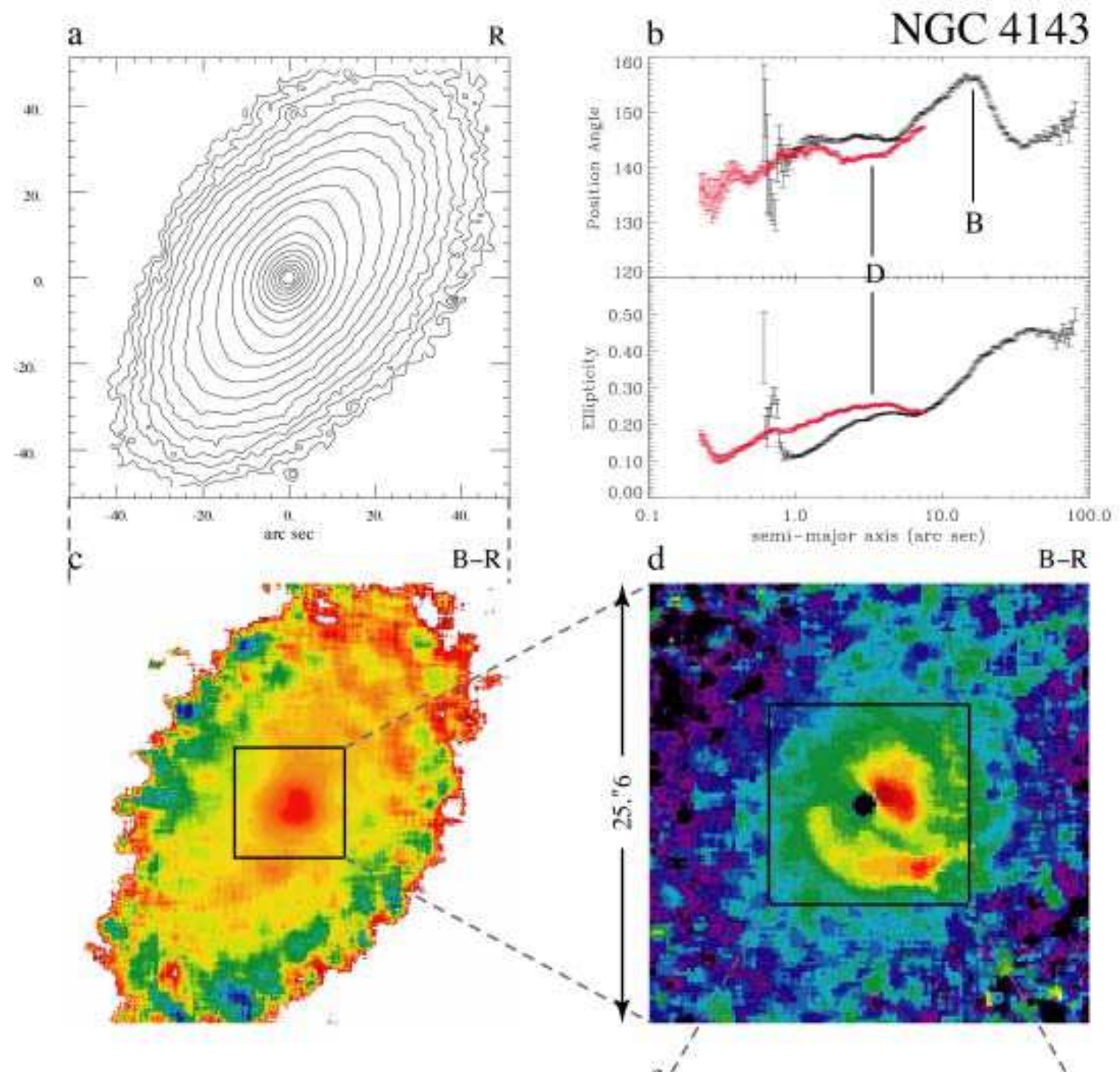

e
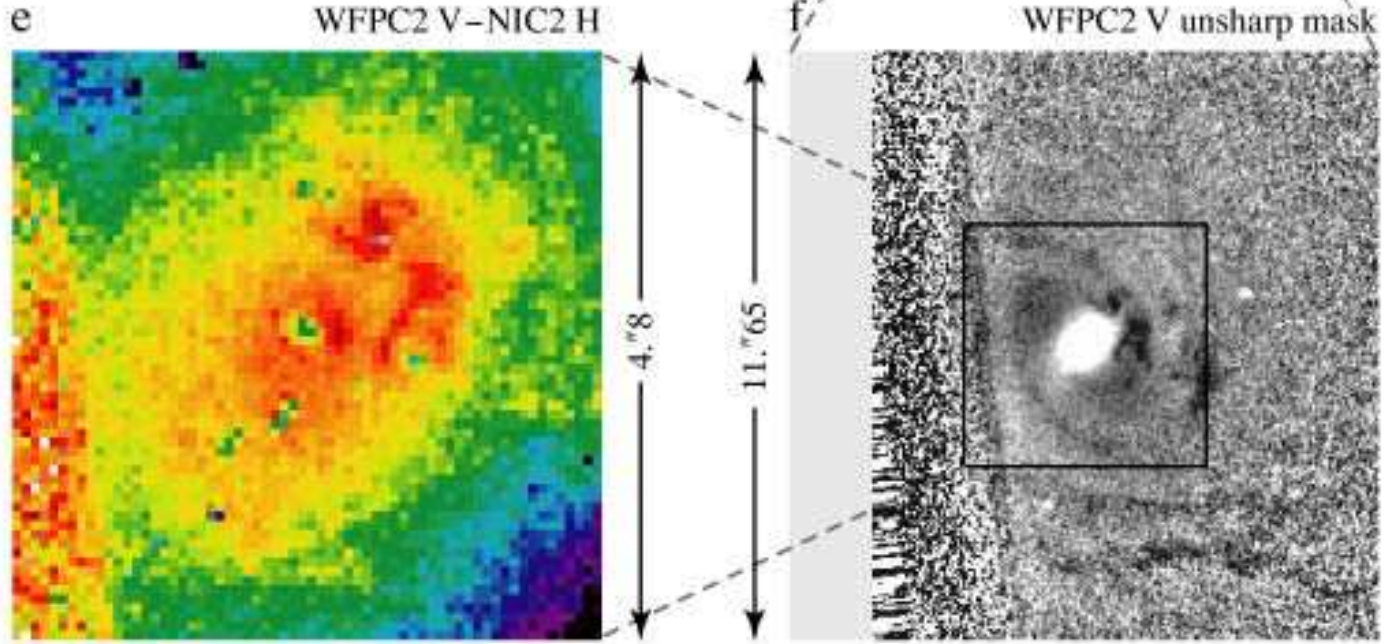

FIG. 26. - NGC 4143: large-scale $R$-band isophotes (a); ellipse fits (b, WIYN $R$ and NICMOS2 F160W [red]); $B-R$ color map on the same scale as panel a $(\mathrm{c}, w=23$, color range within the disk $=0.44 \mathrm{mag})$; small-scale $B-R$ color map $(\mathrm{d}, w=5$, color range $=0.31 \mathrm{mag}$, excluding the saturated center); WFPC2 + NICMOS2 F606W - F160W color map (e, color range in central region $=0.34$ mag); and $\sigma=10$ unsharp mask of the WFPC2 F606W image (f). Note that the galaxy nucleus is near the edge of the PC2 chip, which affects both (e) and (f). The blue nucleus in (d) is an artifact of a saturated $R$-band image. 


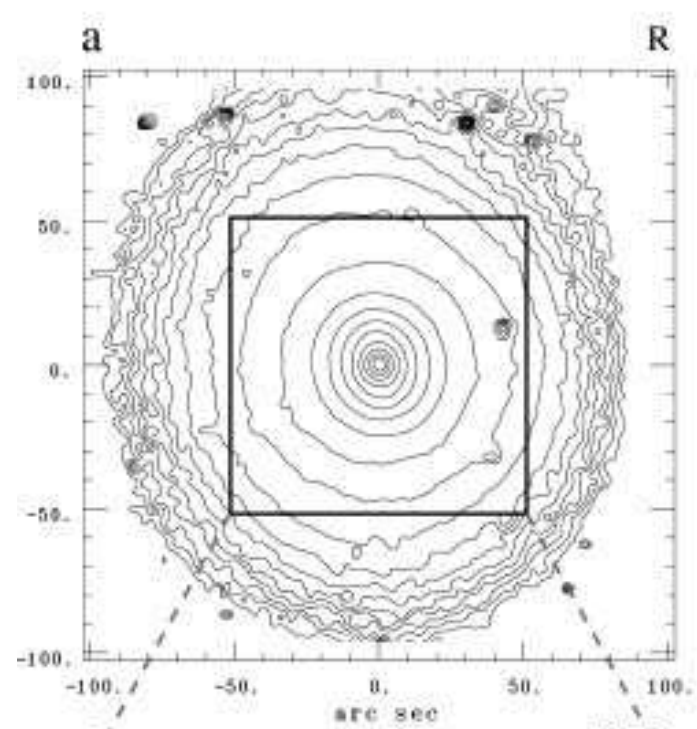

R
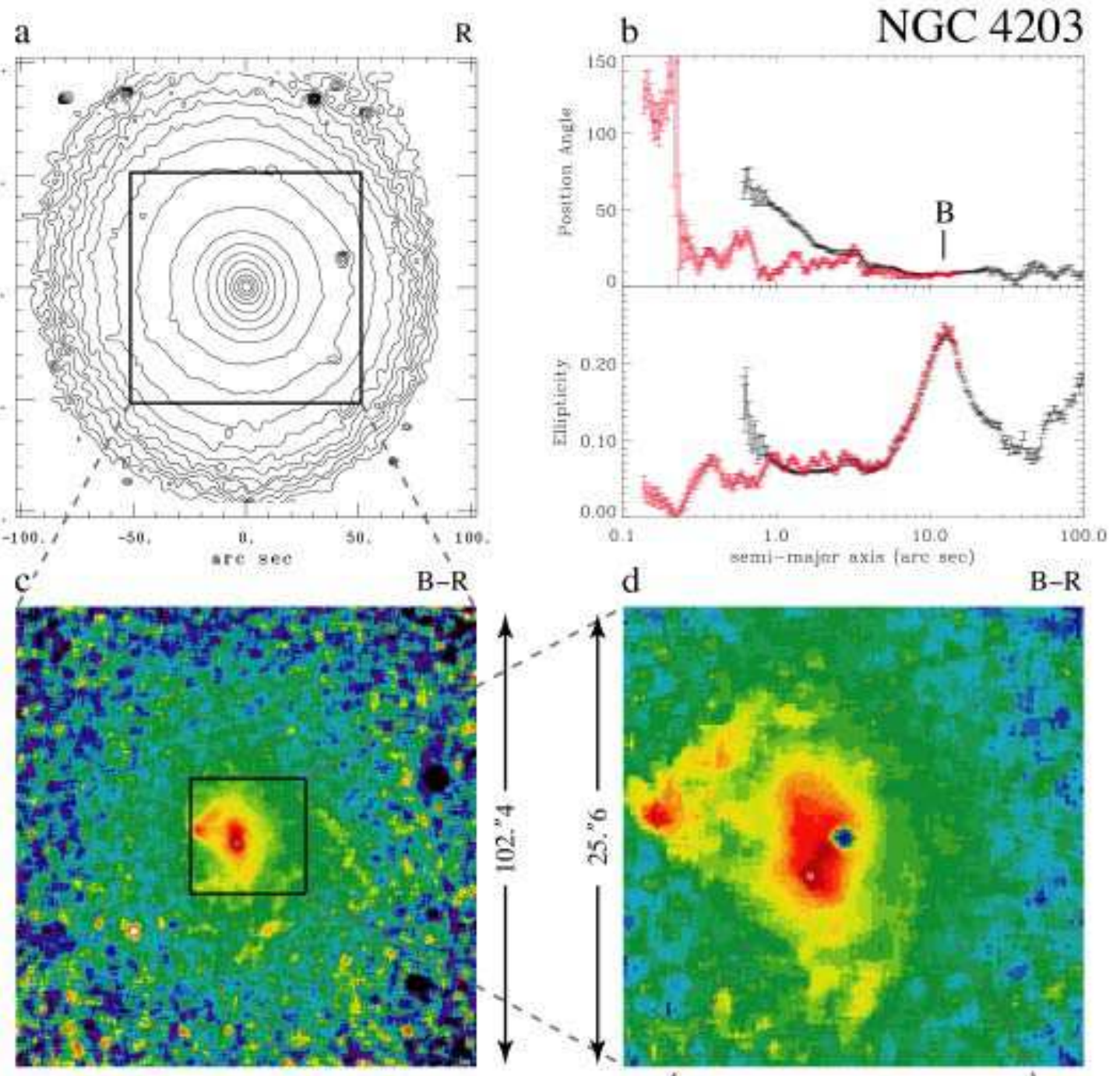

d B-R

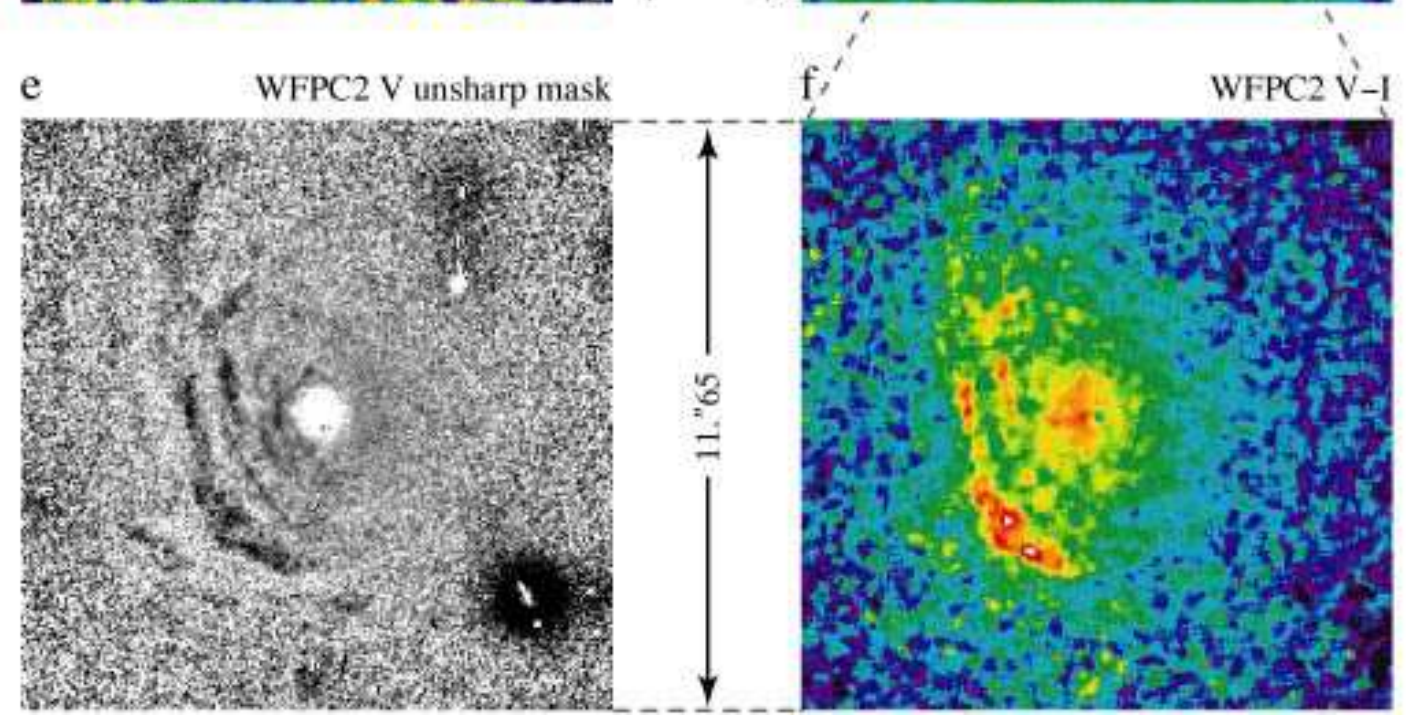

FIG. 27.- NGC 4203: large-scale $R$-band isophotes (a); ellipse fits (b, WIYN $R$ and WFPC2 F814W [red]); $B-R$ color map on two scales $(\mathrm{c}, w=11$, color range $=0.76 \mathrm{mag} ; \mathrm{d}, w=5$, color range $=0.32 \mathrm{mag}) ; \sigma=10$ unsharp mask of the WFPC2 F555W image (e); and WFPC2 F555W - F814W color map (f, $w=5$, color range $=1.34 \mathrm{mag}$ ). Note that the blue nucleus in (d) is an artifact of saturation in the $R$-band image; the blue nucleus in the WFPC2 color map (f) is real. 


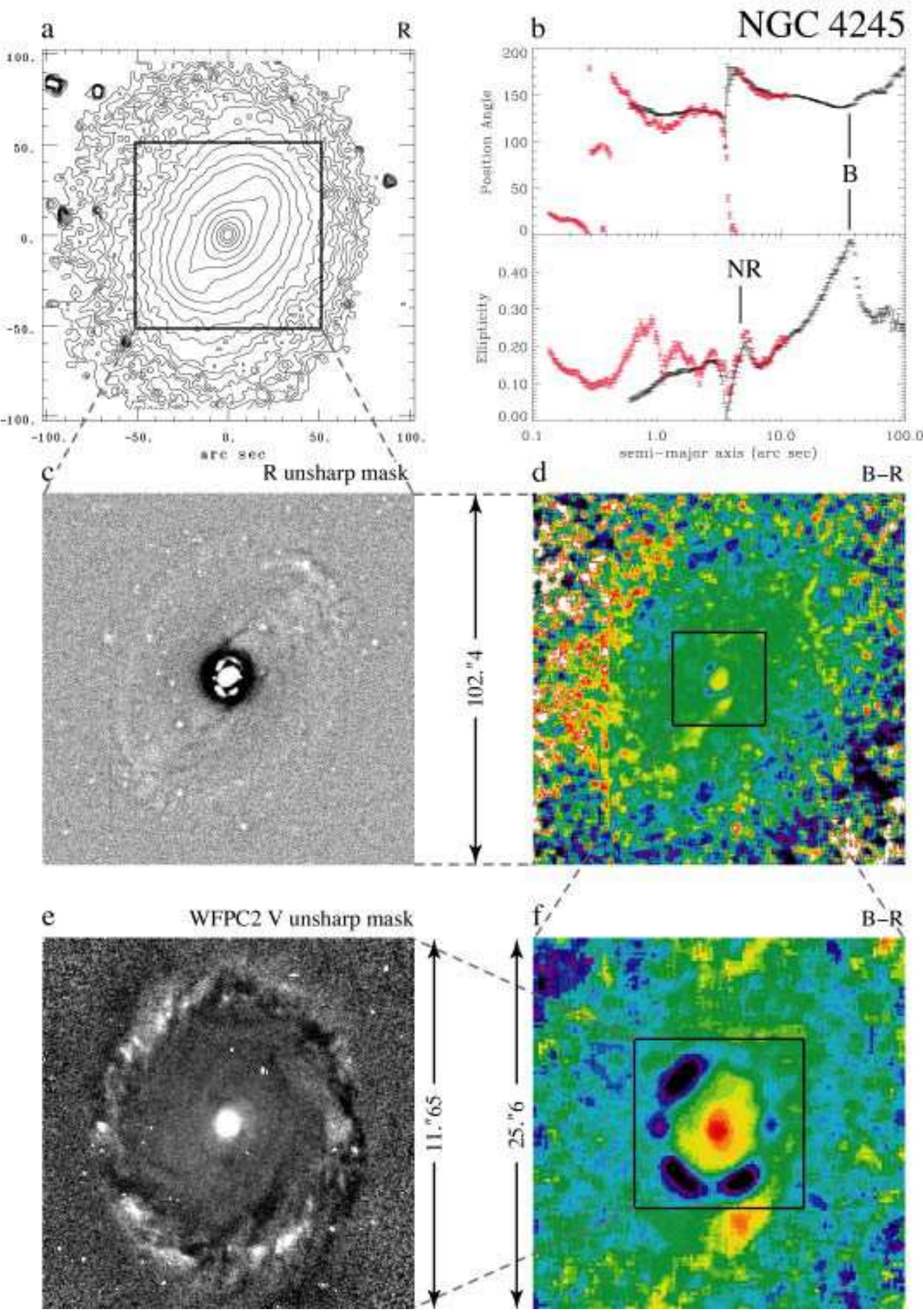

FIG. 28. - NGC 4245: $R$-band isophotes (a); ellipse fits (b, WIYN $R$ and WFPC2 F606W [red]); unsharp mask $(\sigma=10)$ of $R$-band image (c); $B-R$ color map on two scales (d, $w=11$, color range $=1.26 \mathrm{mag} ; \mathrm{f}, w=5$, color range $=0.53 \mathrm{mag}$ ); $\sigma=10$ unsharp mask of the WFPC2 F606W image (e). 

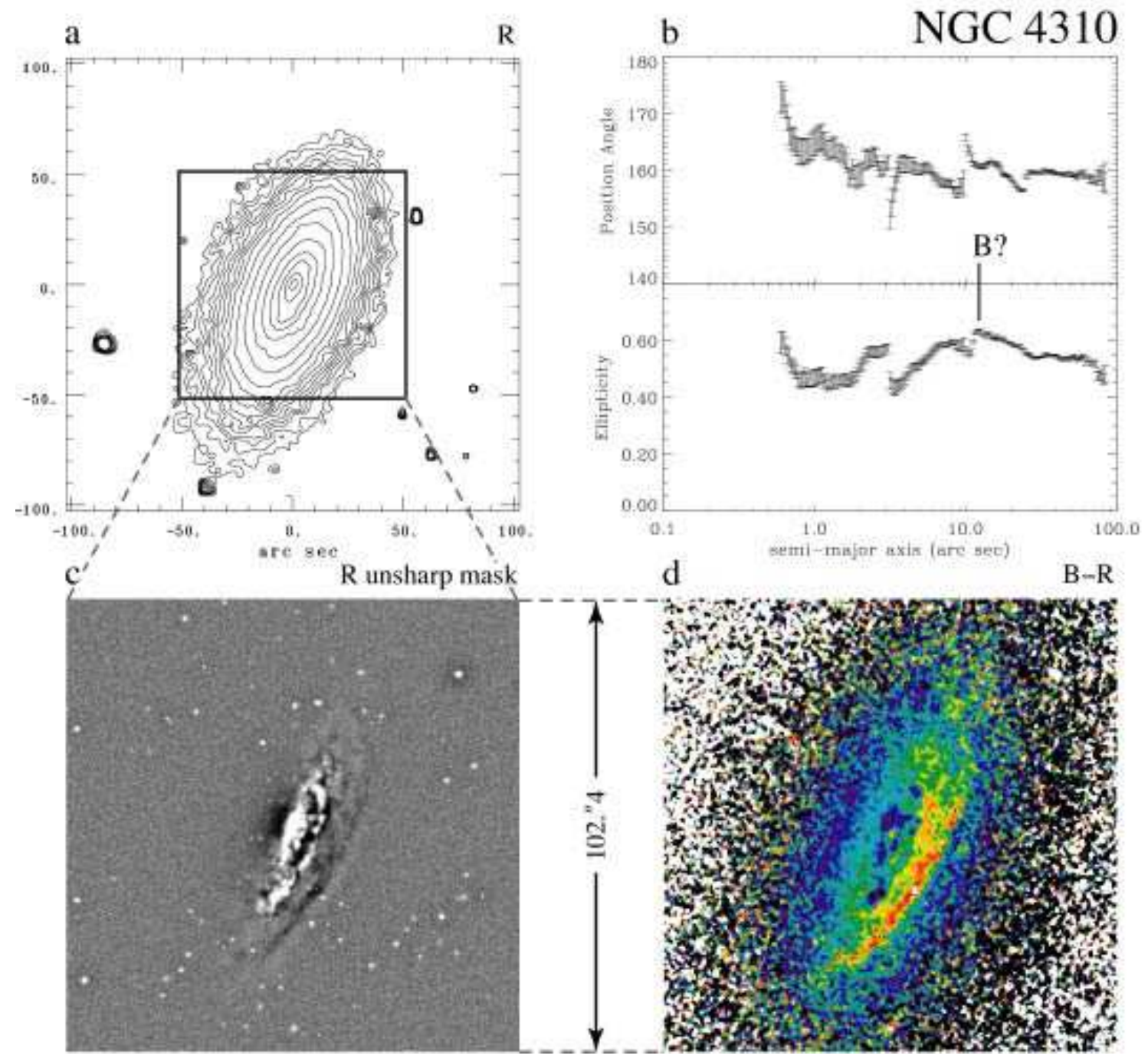

FIG. 29.- NGC 4310: $R$-band isophotes (a); ellipse fits (b, WIYN $R$ ); unsharp mask of $R$-band image (c, $\sigma=10$ ); $B$ - $R$ color map (d, $w=5$, color range $\approx 1.0 \mathrm{mag})$. 

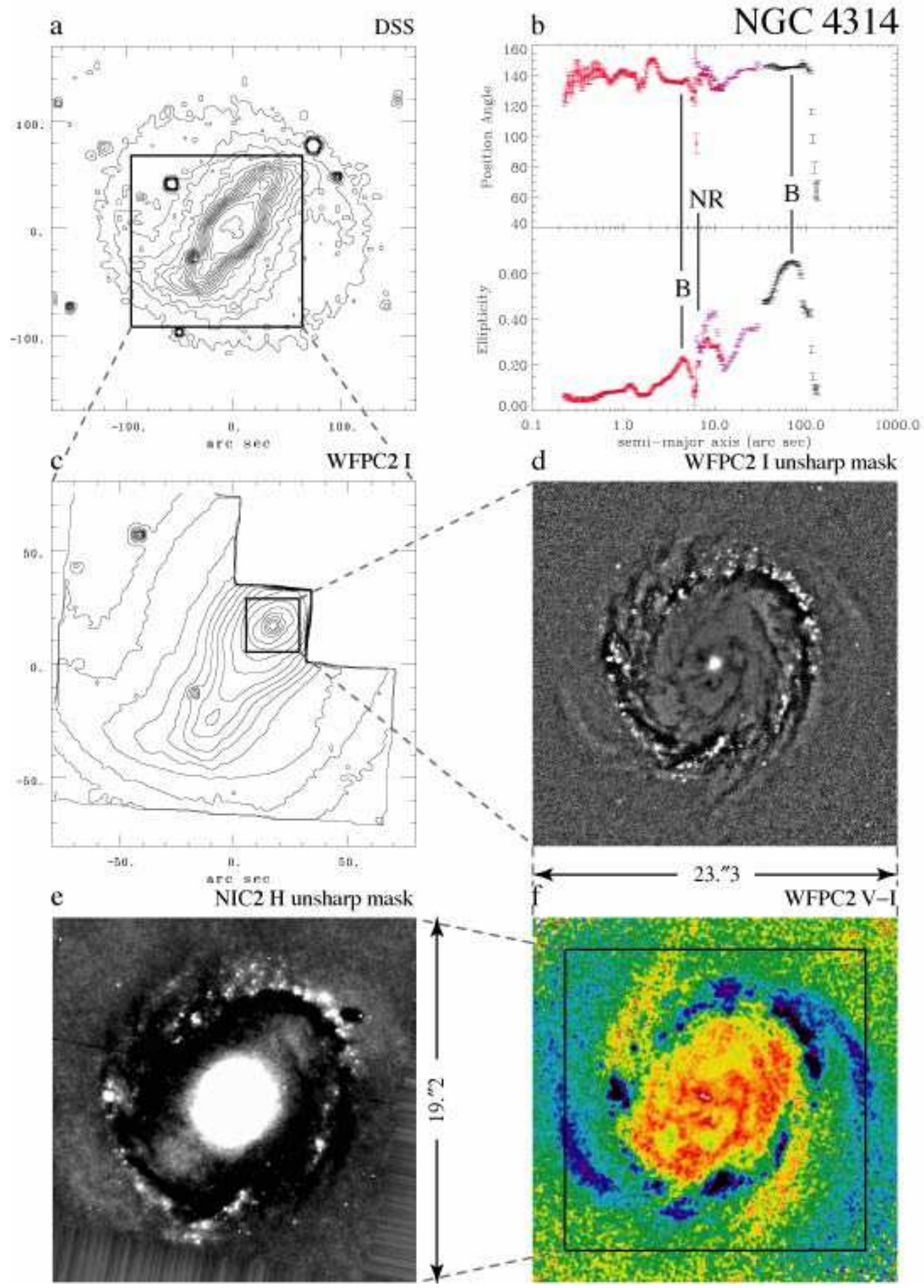

FIG. 30.- NGC 4314: DSS contours (a); ellipse fits (b, DSS [black], WFPC2 F814W mosaic [purple], and NICMOS2 F160W [red]); WFPC2 F814W mosaic contours (c); $\sigma=$ unsharp mask of PC2 F814W image (d); $\sigma=30$ unsharp mask of NICMOS2 F160W image; PC2 $\mathrm{F} 439 \mathrm{~W}-\mathrm{F} 814 \mathrm{~W}$ color map $(\mathrm{f}, w=5$, color range $=2.06 \mathrm{mag}$ ). 

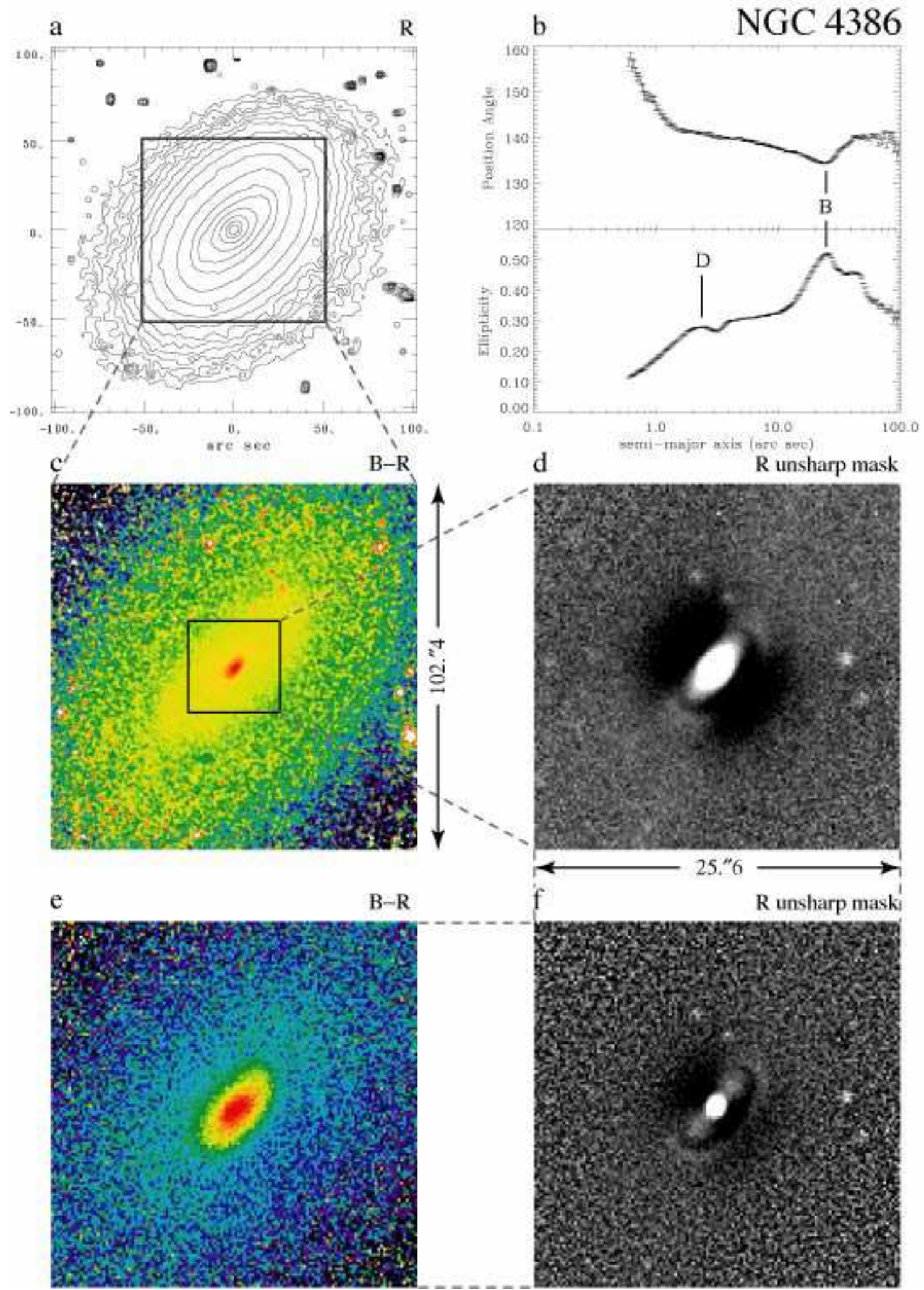

Fig. 31. - NGC 4386: $R$-band isophotes (a); ellipse fits (b, WIYN $R$ ); $B-R$ color maps (c, $w=5$, color range $\approx 2.25$ mag between outer disk and central red disk; e, unsmoothed, color range $\approx 0.71 \mathrm{mag})$; unsharp masks of $R$-band image $(\mathrm{d}, \sigma=5 ; \mathrm{f}, \sigma=2)$. 

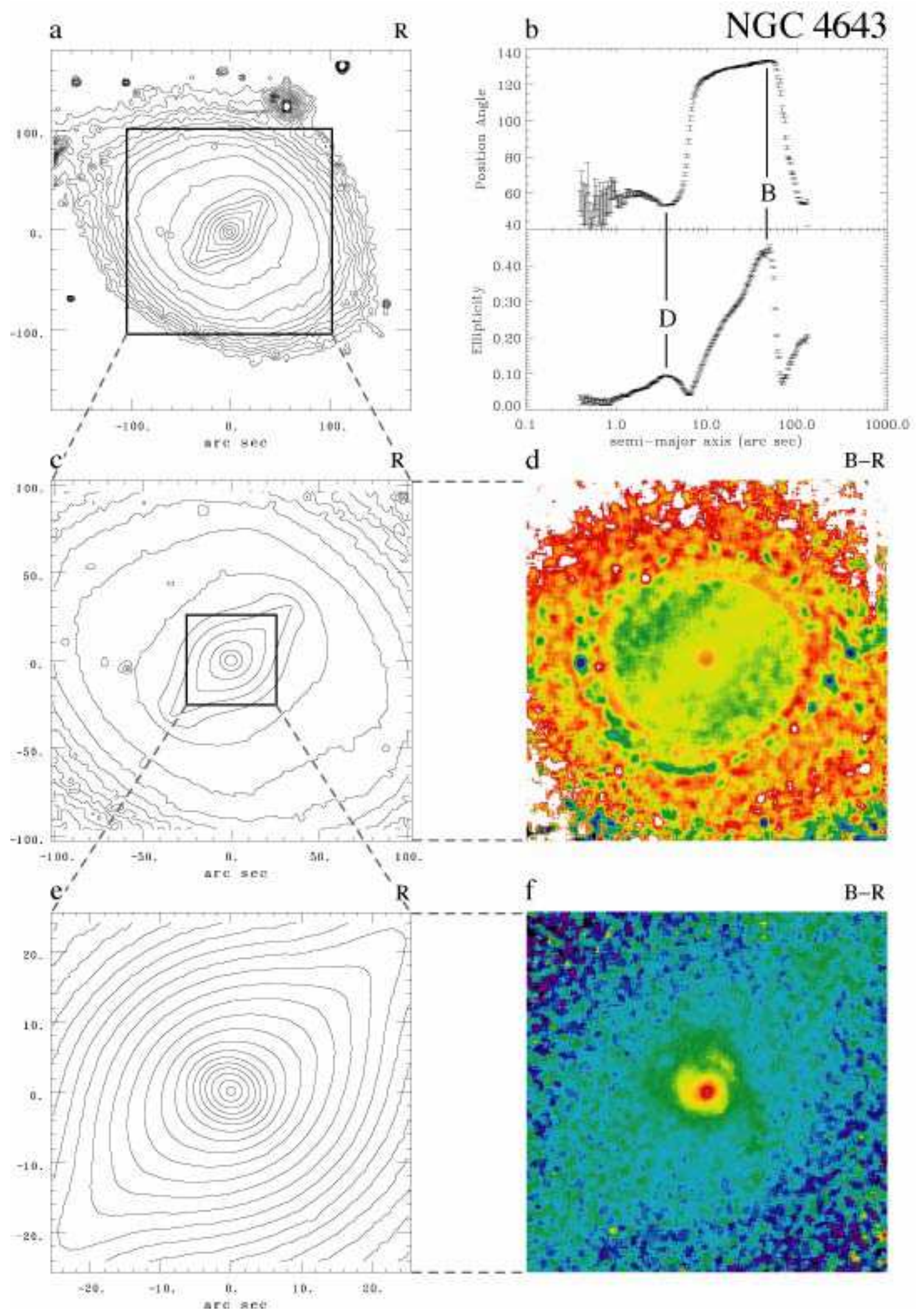

FIG. 32.- NGC 4643: $R$-band isophotes on three scales (a, c, e); ellipse fits (b, WIYN $R$ ); $B-R$ color map on two scales $(\mathrm{d}, w=23$, color range $\approx 0.9 \mathrm{mag} ; \mathrm{f}, w=5$, color range $=0.30 \mathrm{mag})$. 

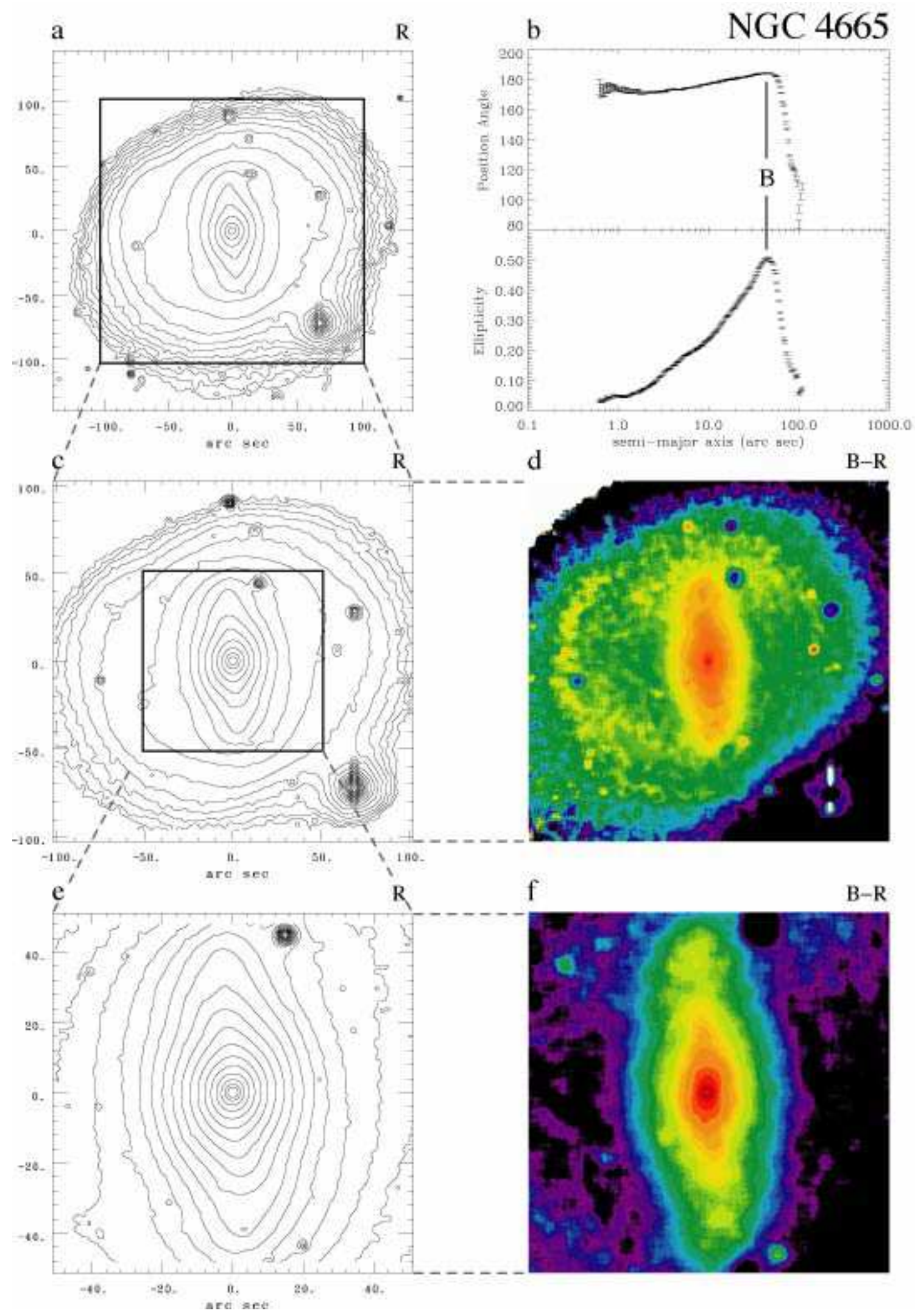

FIG. 33. - NGC 4665: $R$-band isophotes on three scales (a, c, e); ellipse fits (b, WIYN $R$ ); $B-R$ color map on two scales $(\mathrm{d}, w=23$, color range $\approx 0.45$ mag between outer disk [green] and center; f, same smoothing width, color range $=0.48 \mathrm{mag}$ ). 


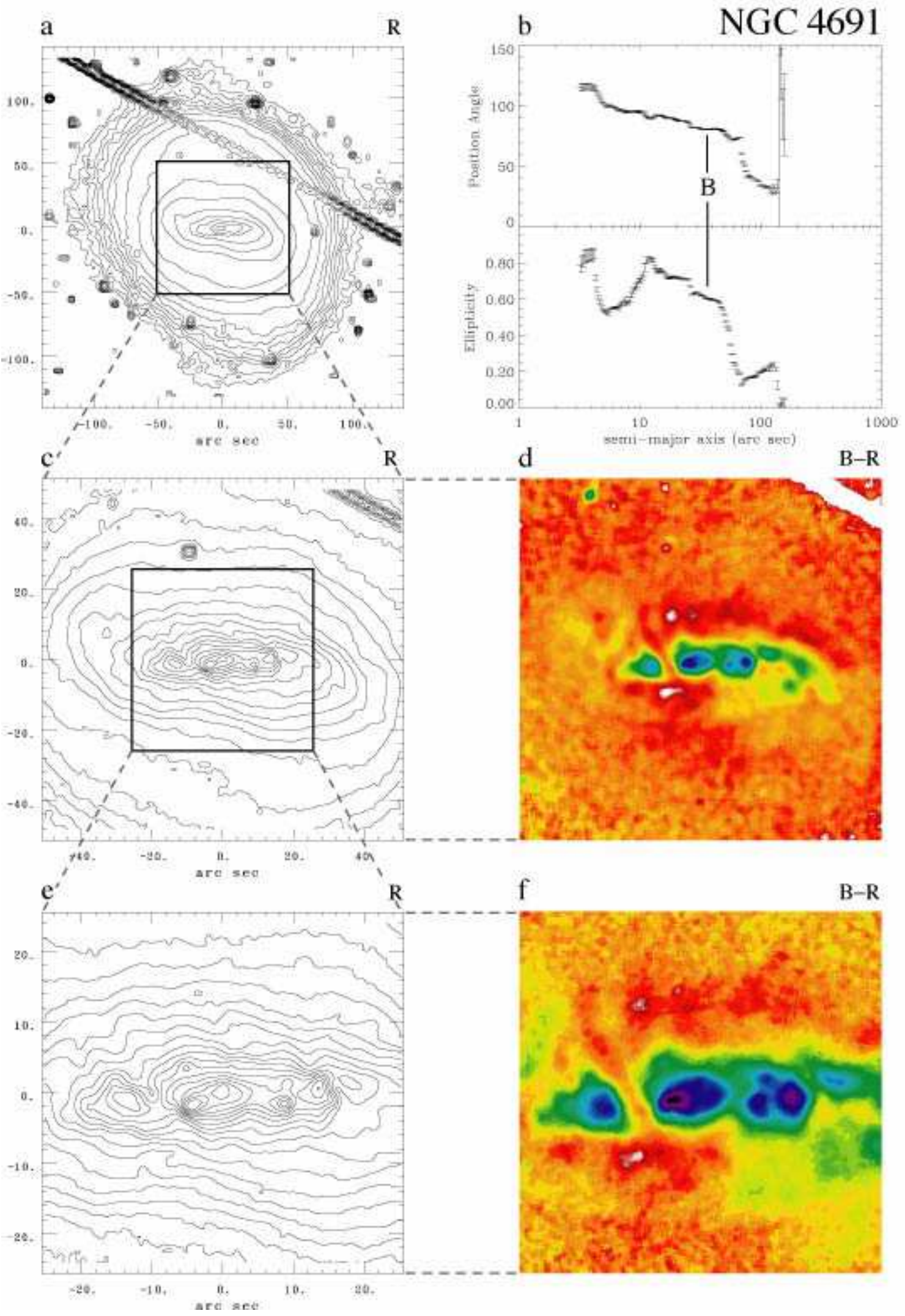

FIG. 34.- NGC 4691: $R$-band isophotes on three scales (a, c, e); ellipse fits (b, WIYN $R$ ); $B-R$ color map on two scales (d, $w=11$, color range $\approx 0.85 \mathrm{mag}$ [excluding the white streak in the NW corner]; $\mathrm{f}, w=5$, color range $=1.02 \mathrm{mag}$ ). The bright streak crossing the northern part of the galaxy (panels a, c, and d) is a satellite or meteor trail. 


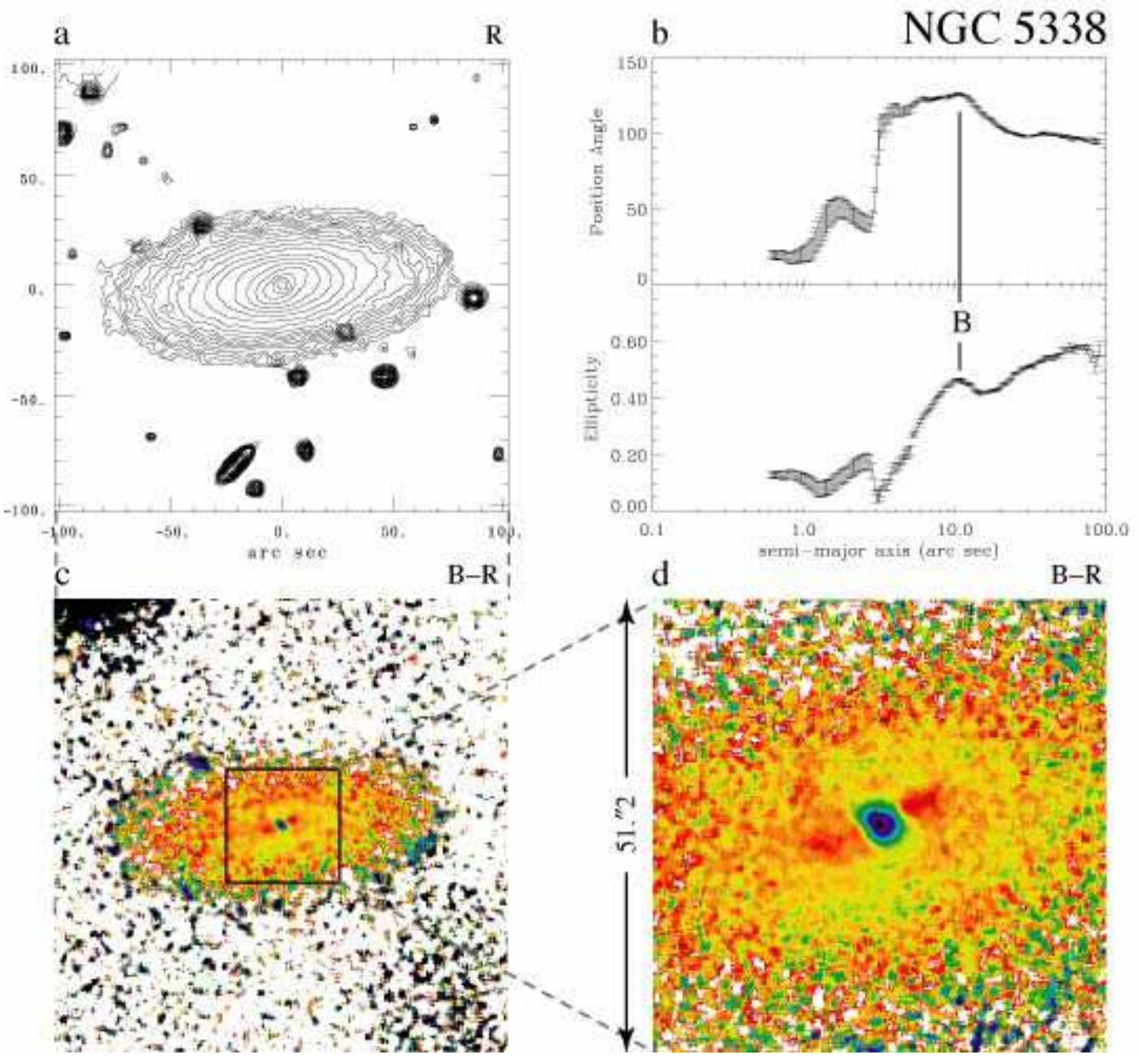

FIG. 35. - NGC 5338: WIYN $R$-band isophotes (a); ellipse fits (b, WIYN $R$ ); $B-R$ color map on two scales $(\mathrm{c}, w=15$, color range $\approx 0.6$ mag; $\mathrm{d}, w=5$, color range $\approx 2.2 \mathrm{mag}, \approx 0.9 \mathrm{mag}$ between center and dust lanes). 

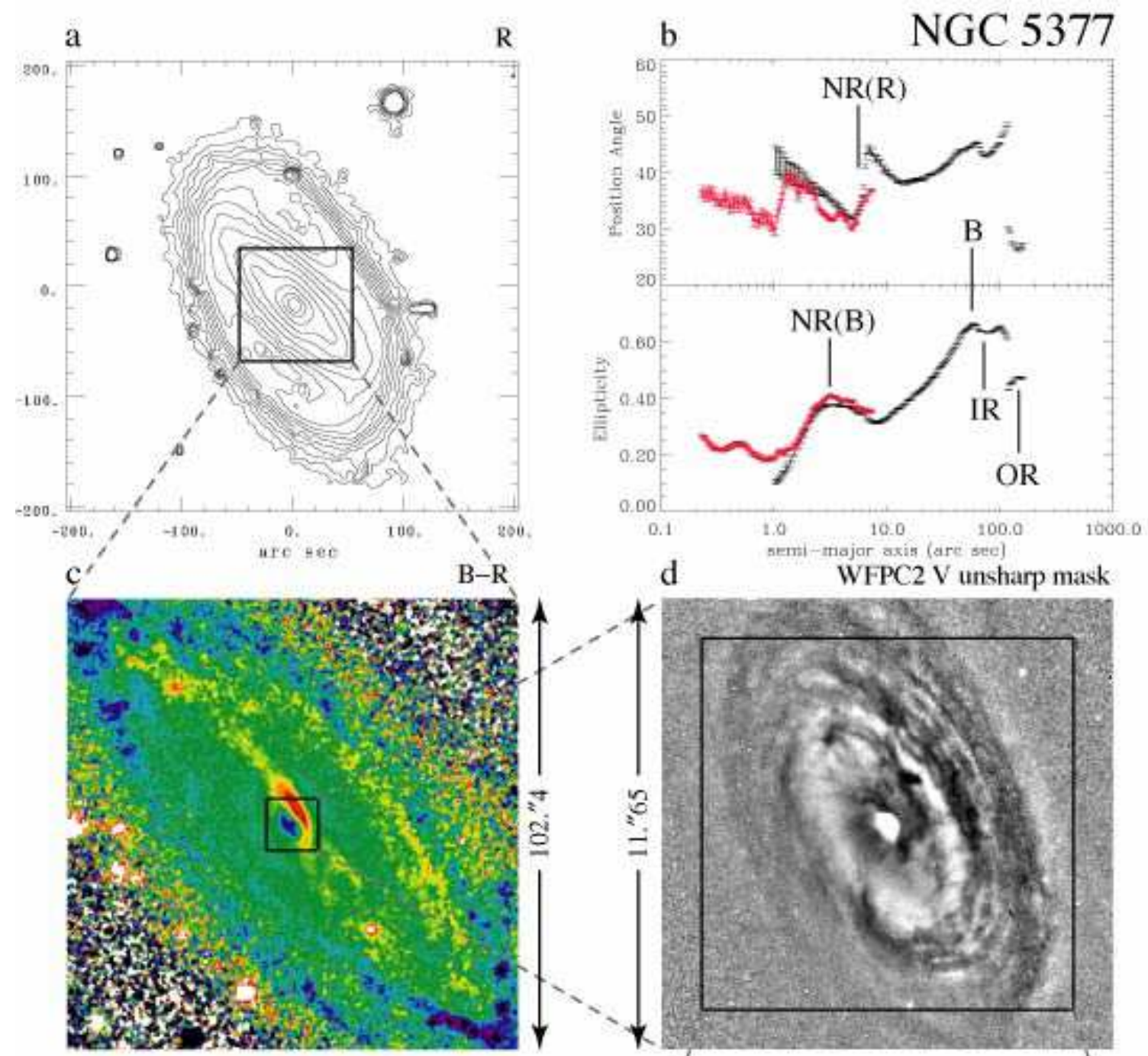

$\mathrm{d}$

WFPC2 V unsharp mask

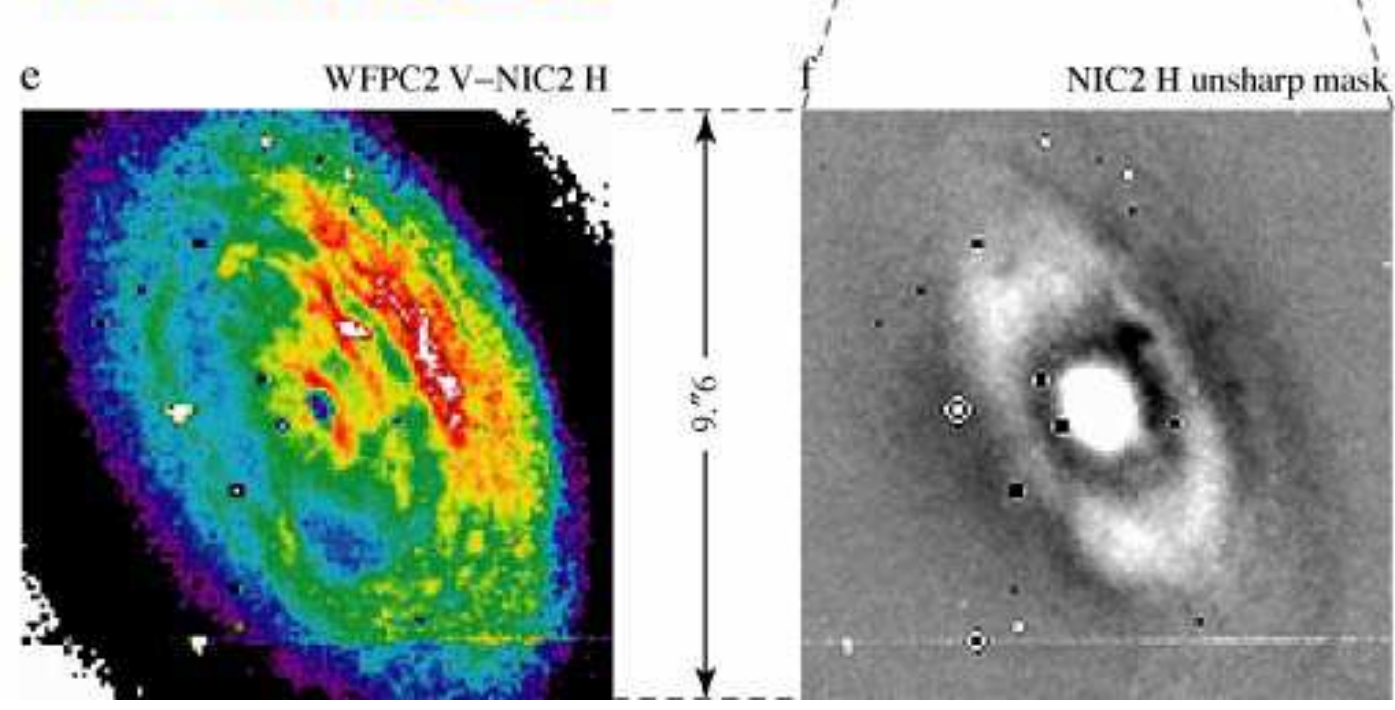

FIG. 36. - NGC 5377: large-scale $R$-band isophotes (a); ellipse fits (b, WIYN $R$ and NICMOS2 F160W [red]); $B-R$ color map (c, $w=5$, color range $=0.81 \mathrm{mag}$ between blue inner ring and red nuclear ring, $=1.44 \mathrm{mag}$ between inner ring and reddest blobs outside); WFPC2 + NICMOS2 $V-H(\mathrm{~F} 606 \mathrm{~W}-\mathrm{F} 160 \mathrm{~W})$ color map (e, unsmoothed, color range $=1.41 \mathrm{mag})$; unsharp masks of $\mathrm{PC} 2 \mathrm{~F} 606 \mathrm{~W}$ image $(\mathrm{d}, \sigma=10)$ and NICMOS2 F160W image (f, $\sigma=10$ ). 


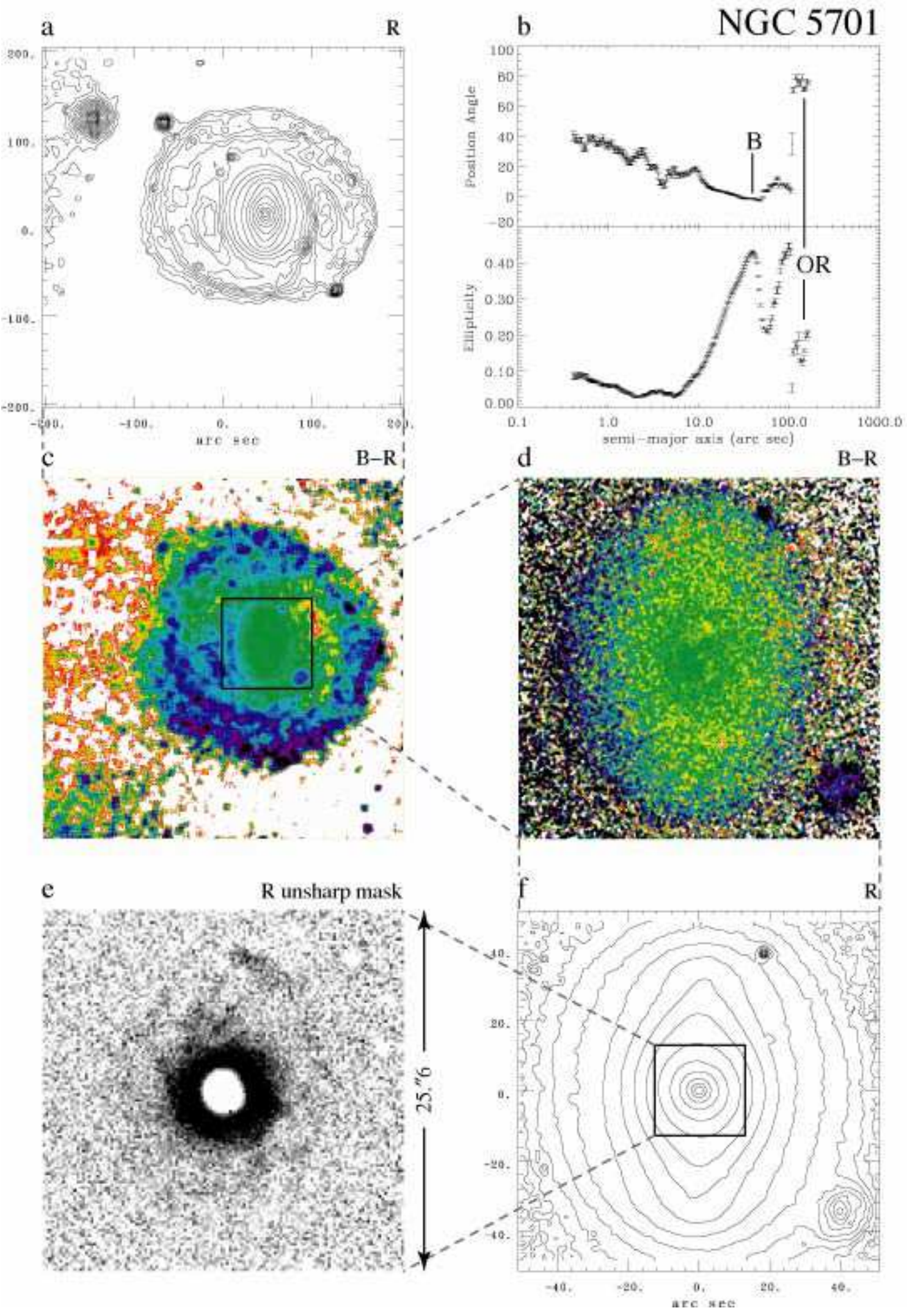

FIG. 37. - NGC 5701: $R$-band isophotes on two scale (a, f); ellipse fits (b, WIYN $R$ ); $B-R$ color maps $(\mathrm{c}, w=41$, color range $=1.44$ mag; $\mathrm{d}, w=5$, color range $=1.43 \mathrm{mag})$; unsharp mask of $R$-band image $(\mathrm{e}, \sigma=5)$. 


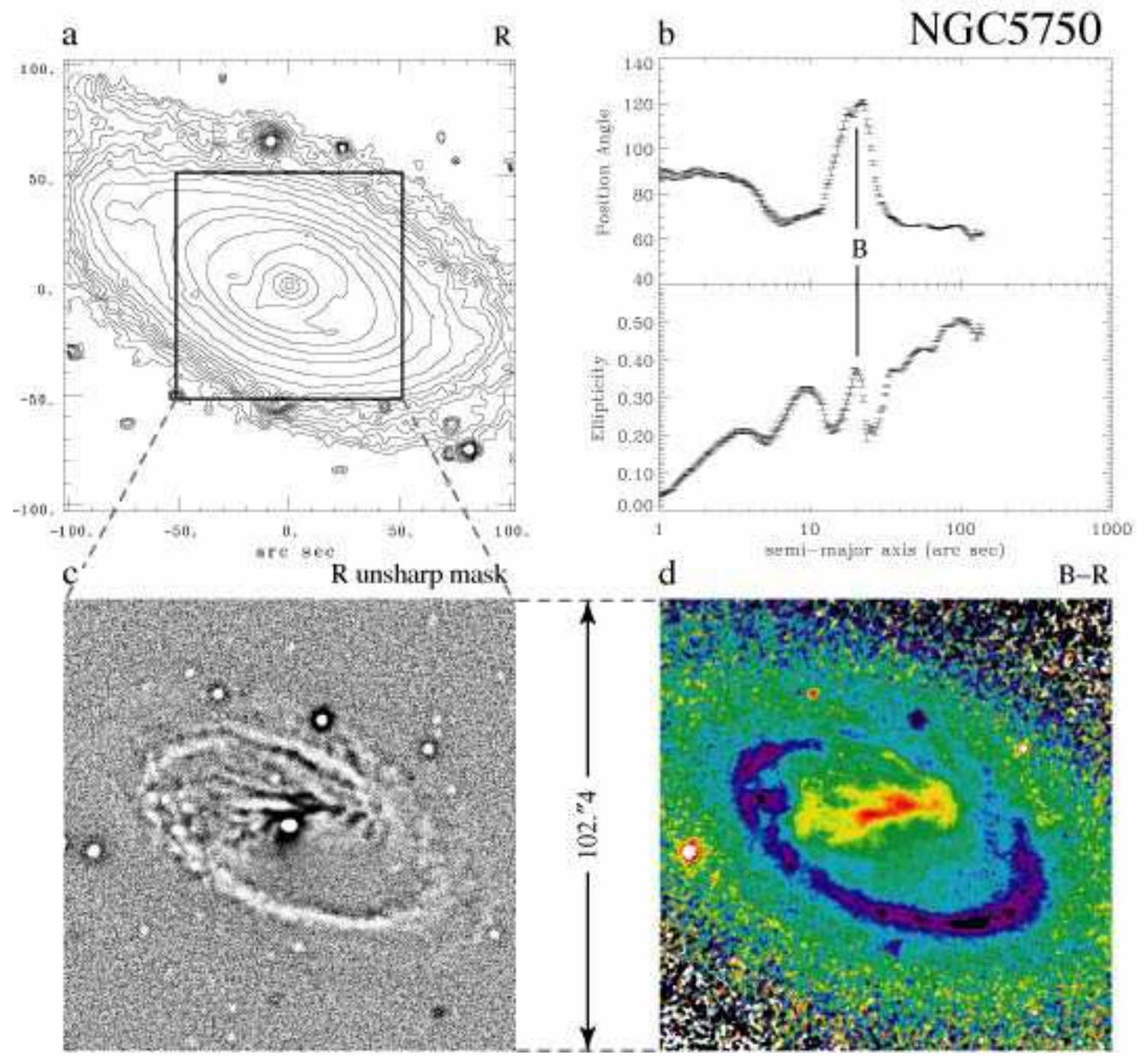

FIG. 38. - NGC 5750: $R$-band isophotes (a); ellipse fits (b, WIYN $R$ ); unsharp mask of $R$-band image (c, $\sigma=5$ ); $B-R$ color map (d, $w=5$, color range $\approx 0.8 \mathrm{mag}$, excluding red star near east edge). 


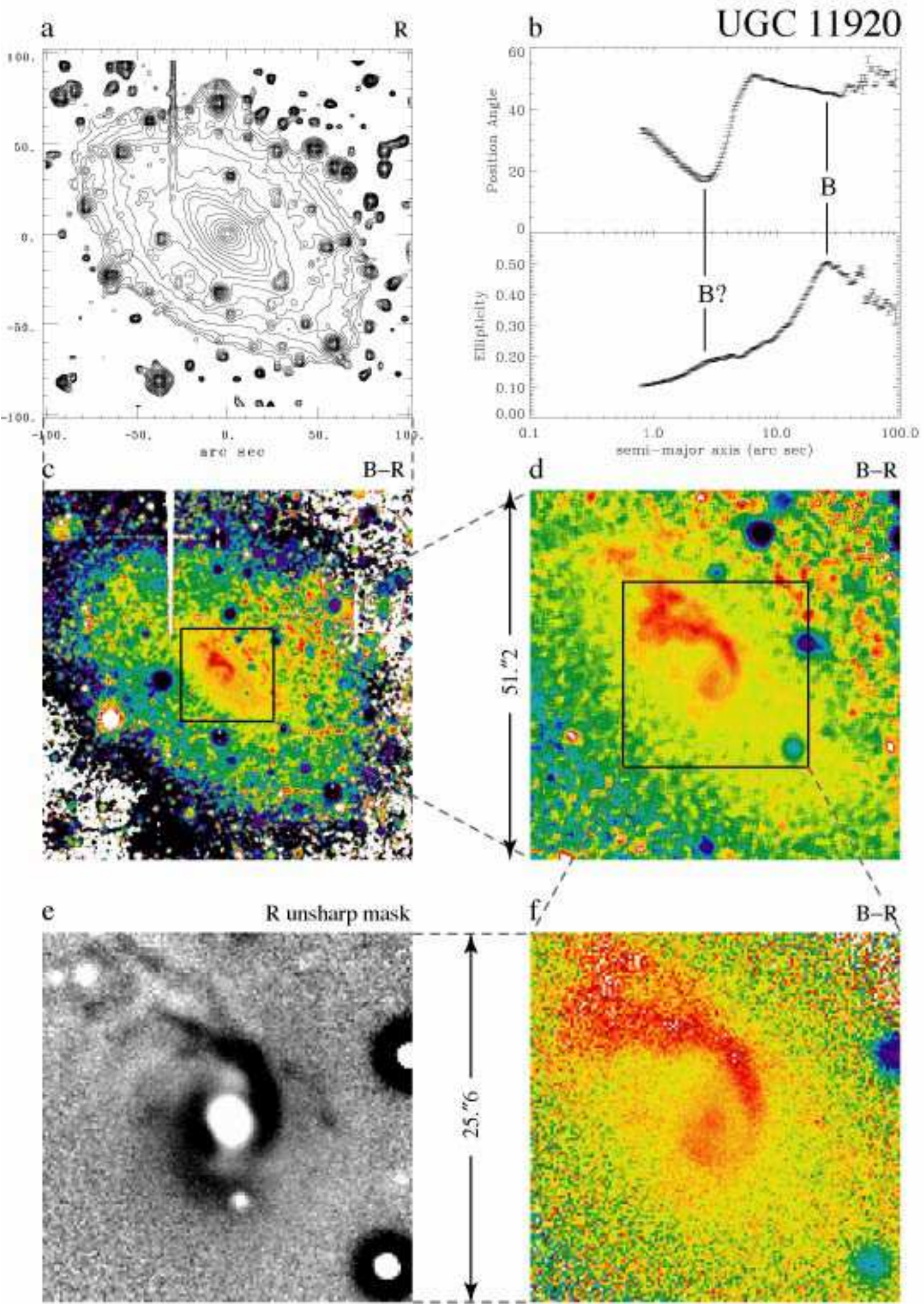

FIG. 39. - UGC 11920: $R$-band isophotes (a, the vertical line east of galaxy center is spillover from a saturated star); ellipse fits (b, WIYN $R$ only); $B-R$ color map (c, $w=11$, color range $\approx 0.3$ mag; $\mathrm{d}, w=5$, color range $=1.15 \mathrm{mag} ; \mathrm{f}$, unsmoothed, color range $\approx 1.15$ mag); unsharp mask of $R$-band image (e, $\sigma=5)$. 

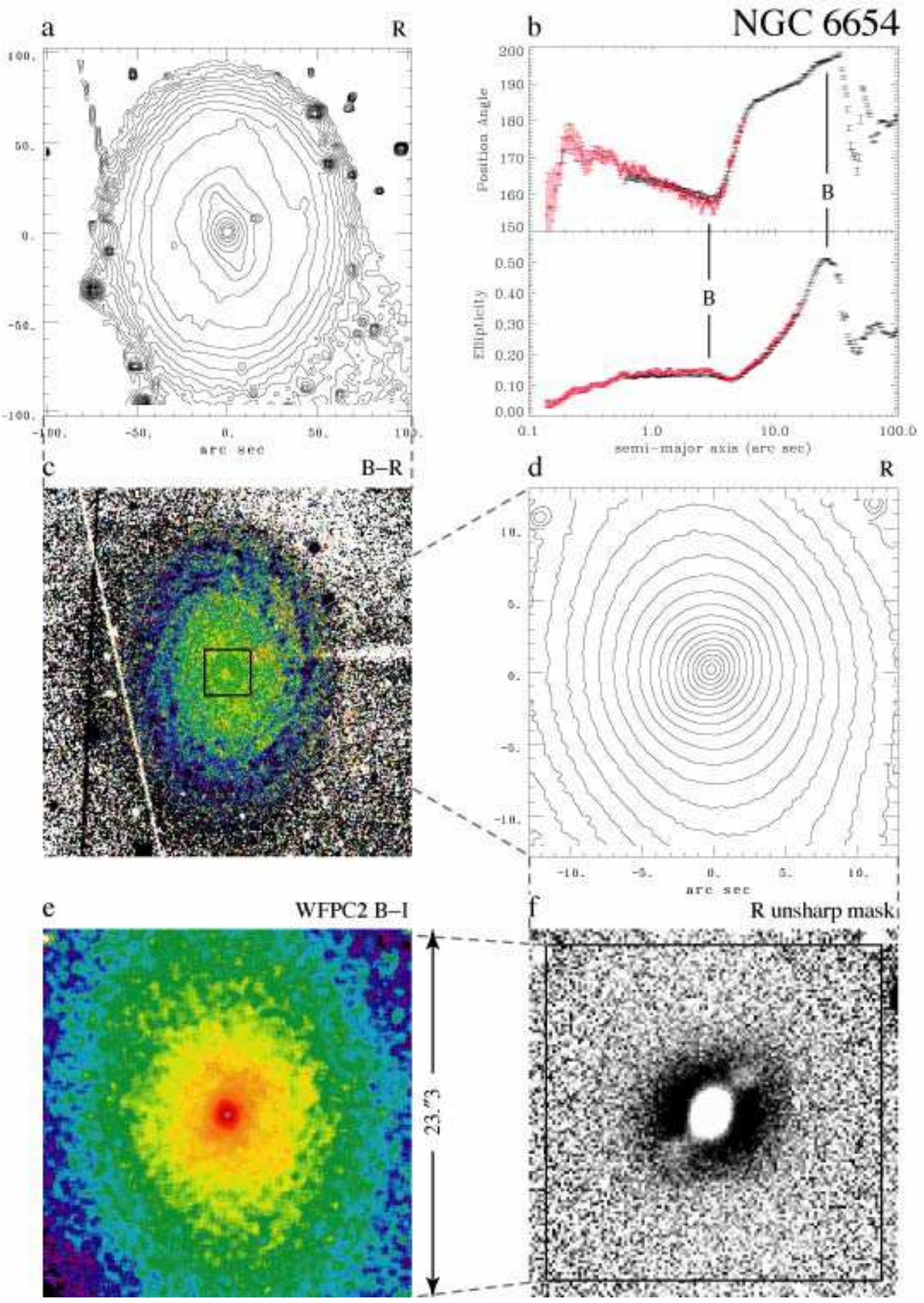

FIG. 40.- NGC 6654: $R$-band isophotes on two scales (a, d); ellipse fits (b, WIYN $R$ and WFPC2 F814W [red]); $B-R$ color map (c, $w=5$, color range $\approx 0.98 \mathrm{mag})$; WFPC2 $\mathrm{F} 450 \mathrm{~W}-\mathrm{F} 814 \mathrm{~W}$ color map $(\mathrm{e}, w=11$, color range $=1.50 \mathrm{mag})$; unsharp mask of $R$-band image (f, $\sigma=5)$. The black and white streaks in the WIYN color map (c) are due to satellite or meteor trails, one of which can be seen in the $R$-band image (a). 


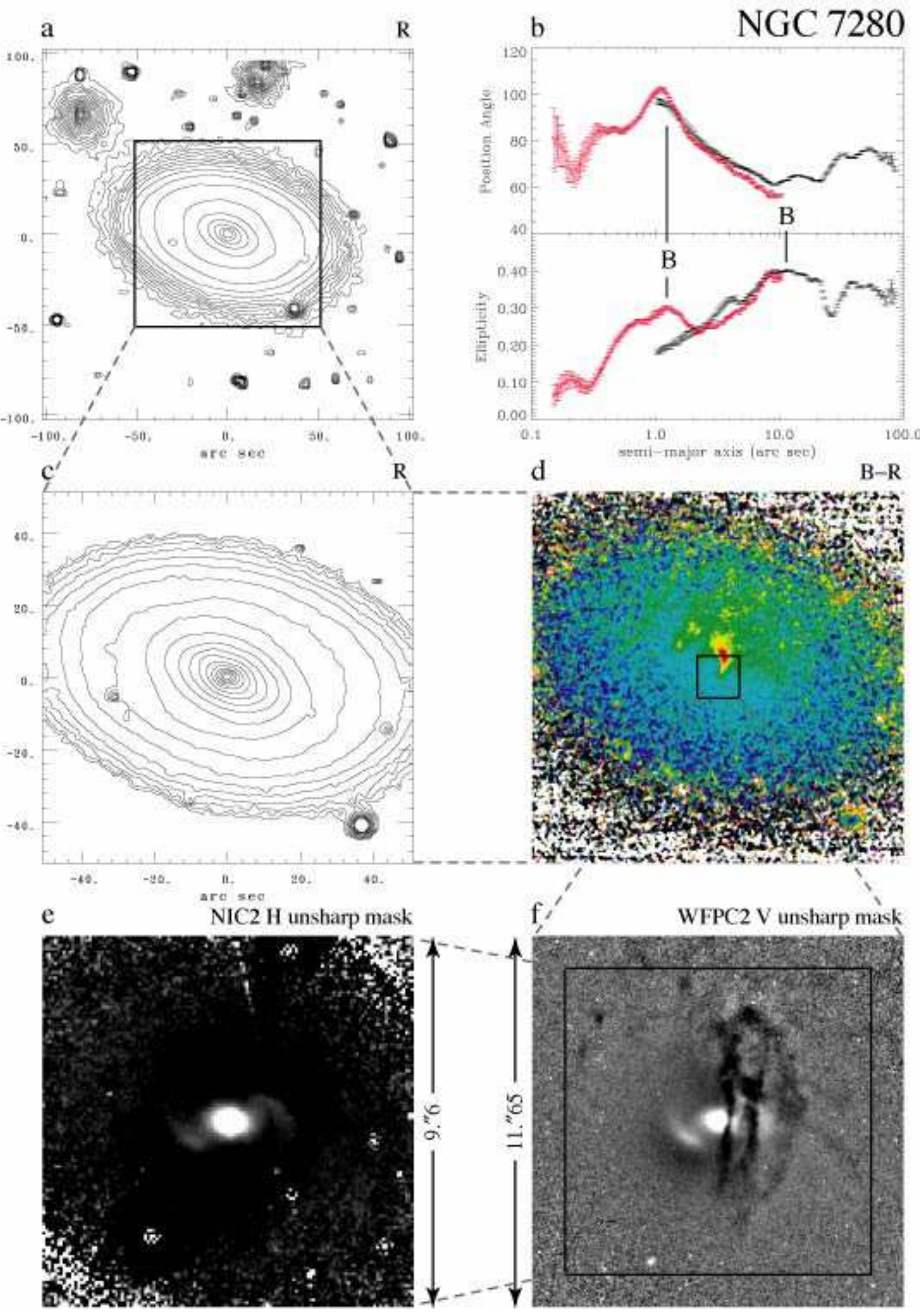

FIG. 41. - NGC 7280: $R$-band isophotes on two scales (a, c); ellipse fits (b, WIYN $R$ and NICMOS2 F160W [red]); $B-R$ color map (d, $w=11$, color range $\approx 0.3 \mathrm{mag} ; \mathrm{f}, w=5$, color range $=1.45 \mathrm{mag}$ ); unsharp masks of NICMOS2 F160W (e, $\sigma=8)$ and WFPC2 F606W (f, $\sigma=10)$ images. 


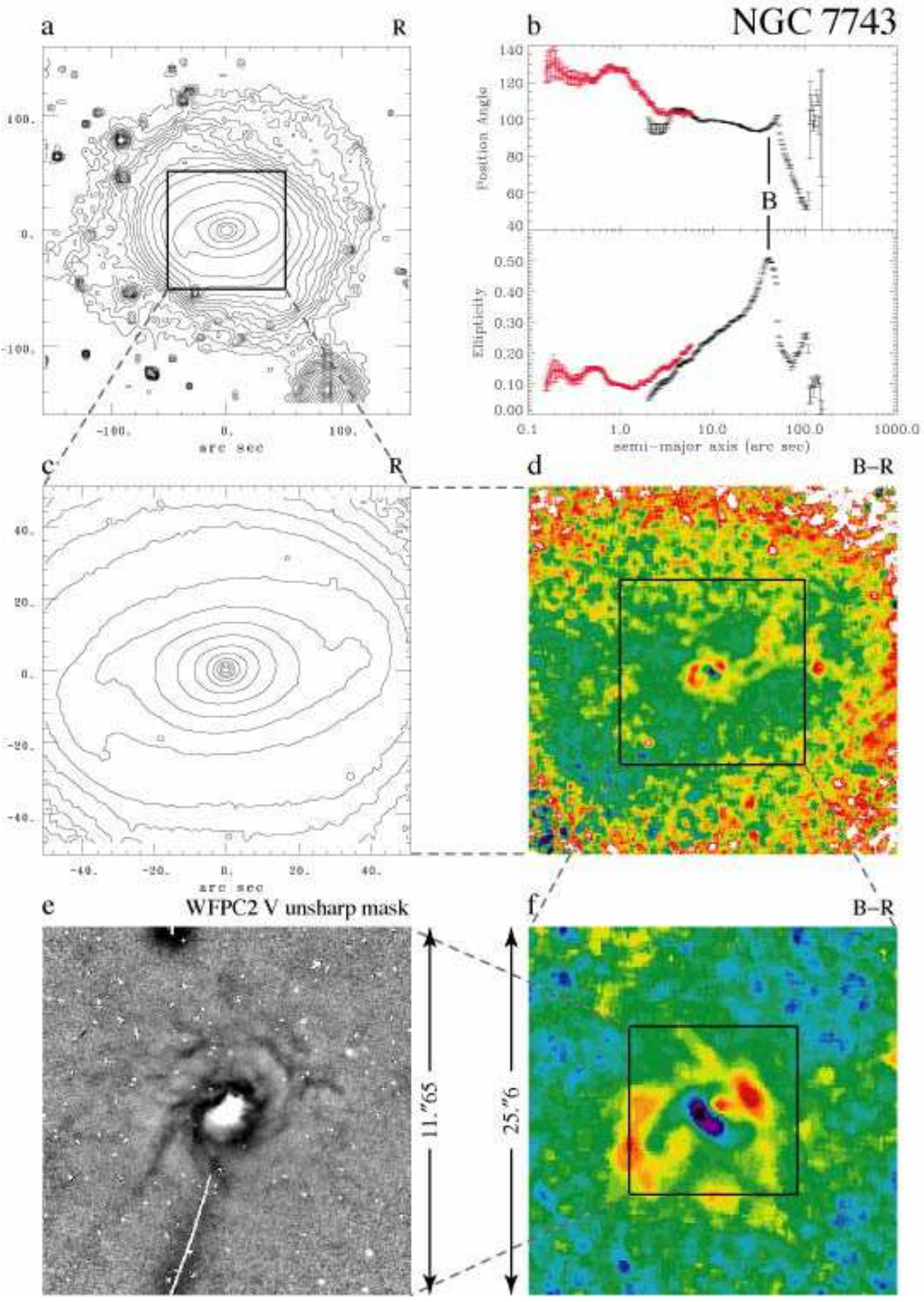

Fig. 42.- NGC 7743: $R$-band isophotes on two scales (a, c; the nuclear isophotes in c are suppressed to show the bar/spiral isophotes more clearly); ellipse fits (b, WIYN $R$ and NICMOS2 F160W [red]); $B-R$ color map on two scales (d, $w=11$, color range $\approx 0.3$ mag; f, $w=5$, color range $=0.31 \mathrm{mag})$; unsharp mask $(\sigma=10)$ of WFPC2 F606W image. 